Review

\title{
Hydrodynamics and phases of flocks
}

\author{
John Toner ${ }^{a}$, Yuhai Tu ${ }^{\text {b,* }}$, Sriram Ramaswamy ${ }^{\mathrm{c}}$ \\ a Institute of Theoretical Science, Department of Physics, University of Oregon, Eugene, \\ OR 97403-5203, USA \\ b IBM T. J. Watson Research Center, P.O. Box 218, Yorktown Heights, NY 10598, USA \\ ${ }^{\mathrm{c}}$ Centre for Condensed Matter Theory, Department of Physics, \\ Indian Institute of Science, Bangalore 560012, India
}

\begin{abstract}
We review the past decade's theoretical and experimental studies of flocking: the collective, coherent motion of large numbers of self-propelled "particles" (usually, but not always, living organisms). Like equilibrium condensed matter systems, flocks exhibit distinct "phases" which can be classified by their symmetries. Indeed, the phases that have been theoretically studied to date each have exactly the same symmetry as some equilibrium phase (e.g., ferromagnets, liquid crystals). This analogy with equilibrium phases of matter continues in that all flocks in the same phase, regardless of their constituents, have the same "hydrodynamic" - that is, long-length scale and long-time behavior, just as, e.g., all equilibrium fluids are described by the Navier-Stokes equations. Flocks are nonetheless very different from equilibrium systems, due to the intrinsically nonequilibrium self-propulsion of the constituent "organisms." This difference between flocks and equilibrium systems is most dramatically manifested in the ability of the simplest phase of a flock, in which all the organisms are, on average moving in the same direction (we call this a "ferromagnetic" flock; we also use the terms "vector-ordered" and "polar-ordered" for this situation) to exist even in two dimensions (i.e., creatures moving on a plane), in defiance of the well-known Mermin-Wagner theorem of equilibrium statistical mechanics, which states that a continuous symmetry (in this case, rotation invariance, or the ability of the flock to fly in any direction) can not be spontaneously broken in a two-dimensional system with only short-ranged interactions. The "nematic" phase of flocks,
\end{abstract}

\footnotetext{
* Corresponding author.

E-mail address: yuhai@us.ibm.com (Y. Tu).
} 
in which all the creatures move preferentially, or are simply oriented preferentially, along the same axis, but with equal probability of moving in either direction, also differs dramatically from its equilibrium counterpart (in this case, nematic liquid crystals). Specifically, it shows enormous number fluctuations, which actually grow with the number of organisms faster than the $\sqrt{N}$ "law of large numbers" obeyed by virtually all other known systems. As for equilibrium systems, the hydrodynamic behavior of any phase of flocks is radically modified by additional conservation laws. One such law is conservation of momentum of the background fluid through which many flocks move, which gives rise to the "hydrodynamic backflow" induced by the motion of a large flock through a fluid. We review the theoretical work on the effect of such background hydrodynamics on three phases of flocks - the ferromagnetic and nematic phases described above, and the disordered phase in which there is no order in the motion of the organisms. The most surprising prediction in this case is that "ferromagnetic" motion is always unstable for low Reynolds-number suspensions. Experiments appear to have seen this instability, but a quantitative comparison is awaited. We conclude by suggesting further theoretical and experimental work to be done.

\section{Introduction}

Flocking [1] - the collective, coherent motion of large numbers of organisms - is one of the most familiar and ubiquitous biological phenomena. We have all seen flocks of birds, schools of fish, herd of wildebeest, etc. (at least on film). We will hereafter refer to all such collective motions_-flocks, swarms, herds, etc.—as "flocking." This phenomenon also spans an enormous range of length scales: from kilometers (herds of wildebeest) to micrometers (e.g., the micro-organism Dictyostelium discoideum) [2-4]. Remarkably, despite the familiarity and widespread nature of the phenomenon, it is only in the last 10 years or so that many of the universal features of flocks have been identified and understood. It is our goal in this paper to review these recent developments, and to suggest some of the directions future research on this subject could take.

This "modern era" in the understanding of flocks began with the work of Vicsek et al. [5], who was, to our knowledge, the first to recognize that flocks fall into the broad category of nonequilibrium dynamical systems with many degrees of freedom that has, over the past few decades, been studied using powerful techniques originally developed for equilibrium condensed matter and statistical physics (e.g., scaling, the renormalization group, etc.). In particular, Vicsek noted an analogy between flocking and ferromagnetism: the velocity vector of the individual birds is like the magnetic spin on an iron atom in a ferromagnet. The usual "moving phase" of a flock, in which all the birds, on average, are moving in the same direction, is then the analog of the "ferromagnetic" phase of iron, in which all the spins, an average, point in the same direction. Another way to say this is that the development of a nonzero mean center of mass velocity $\langle\vec{v}\rangle$ for the flock as a whole therefore requires spontaneous breaking of a continuous symmetry (namely, rotational), precisely as the development of a nonzero magnetization $\vec{M} \equiv\langle\vec{S}\rangle$ of the spins in a ferromagnet breaks the continuous [6] spin rotational symmetry of the Heisenberg magnet [7]. 
Of course, many flocking organisms do not move in a rotation-invariant environment. Indeed, the most familiar examples of flocking - namely, the seasonal migrations of birds and mammals - clearly do not: creatures move preferentially south (in the Northern hemisphere) as winter approaches, and north, as summer does. Presumably, the individual organisms get their directional cues from their environment - the sun, wind, ocean and air currents, temperature gradients, the earth's magnetic field, and so on - by a variety of means which we will term "compasses" [8]. Collective effects of the type we will focus most of our attention on in this paper are in principle less important in such "directed flocks." However, collective aligning tendencies greatly enhance the ability of the flock to orient in the presence of a small external guiding field. Once aligned, like a permanent magnet made of soft iron, a flock below its ordering "temperature" will stay aligned even without the guiding field. Moreover, even if there are external aligning fields, it is highly likely that creatures in the interior of a flock decide their alignment primarily by looking at their immediate neighbors, rather than the external field. Gruler et al. [9] has remarked on the possible physiological advantage of the spontaneous aligning tendency. We will have little more to say about this here (but see the discussion in Section 8).

All in all, it seems likely that there are many examples of flocking in nature where collective behavior and interparticle interaction dominate over externally imposed aligning fields, and experimental situations can certainly be devised where the gradients of nutrient, temperature or gravitational potential that produce such nonspontaneous alignment are eliminated, enabling a study of the phenomenon of spontaneous order in flocks of micro-organisms such as $D$. discoidae [2] and melanocytes [9], the critters that carry human skin pigment. It is precisely here, as Vicsek noted, that the ferromagnetic analogy just described immediately becomes useful, suggesting that compasses are not necessary to achieve a coherently moving "ferromagnetic" flock, just as no external magnetic field picking out a special direction for the spins is necessary to produce spontaneous magnetization in ferromagnets. It now becomes an interesting question whether or not such spontaneous long-ranged order-by which we mean order (in this case, collective motion of all of the birds in the same direction) that arises not by being imposed by an external field detected by an internal compass, but rather arises just from the interaction of the birds with each other - can, in fact, occur in flocks as it does in ferromagnets [10]. Is the analogy to ferromagnets truly a good one?

Vicsek pushed this analogy much further. Just as the spins in ferromagnets only have short-ranged interactions (often modeled as strictly nearest-neighbor) so birds in a flock may only interact with a few nearest neighbors. Of course, one again might dispute this idea: perhaps birds can see the flock as a whole, and respond to its movements. While this is undoubtedly true of some organisms (e.g., many types of birds), it again seems unlikely that all flocking organisms (particularly microscopic ones) have such long-ranged interactions. And likewise it is again an interesting question whether such interactions are necessary to achieve a ferromagnetic flocking state.

There remains one further analogy between moving flocks and ferromagnets: temperature. The most striking thing about long-ranged ferromagnetic order in systems 
with only short-ranged interactions is that it is robust at finite temperature, a fact so un-obvious that it was not firmly established until Onsager's solution of the 2D Ising model. Is there an analogy of temperature in flocks? Vicsek realized that there was: errors made by the birds as they tried to follow their neighbors. The randomness of these errors introduces a stochastic element to the flocking problem in much the same way that thermal fluctuations do at nonzero temperature in an equilibrium ferromagnet. Does the ordered, coherently moving "ferromagnetic" state of a flock survive such randomness, making a uniformly moving, arbitrarily large flock possible, just as an arbitrarily large chunk of iron can become uniformly magnetized, even at finite temperature (and, indeed, is in its ordered, ferromagnetic phase at room temperature)?

To answer this, and the questions raised earlier, about the nature of, and requirements for, flocking, Vicsek devised a "minimal" numerical simulation model for flocking. The model incorporates the following general features:

1. A large number (a "flock") of point particles ("boids" [11]) each move over time through a space of dimension $d(=2,3, \ldots)$, attempting at all times to "follow" (i.e., move in the same direction as) its neighbors.

2. The interactions are purely short-ranged: each "boid" only responds to its neighbors, defined as those "boids" within some fixed, finite distance $R_{0}$, which is assumed to be independent of $L$, the linear size of the "flock."

3. The "following" is not perfect: the "boids" make errors at all times, which are modeled as a stochastic noise. This noise is assumed to have only short-ranged spatio-temporal correlations.

4. The underlying model has complete rotational symmetry: the flock is equally likely, a priori, to move in any direction.

Any model that incorporates these general features should belong to the same "universality class," in the sense that term is used in critical phenomena and condensed matter physics. The specific discrete-time model proposed and simulated numerically by Vicsek is the following:

The $i$ th bird is situated at position $\left\{\vec{r}_{i}(t)\right\}$ in a two-dimensional plane, at integer time $t$. Each chooses the direction it will move on the next time step (taken to be of duration $\Delta t=1$ ) by averaging the directions of motion of all of those birds within a circle of radius $R_{0}$ (in the most convenient units of length $R_{0}=1$ ) on the previous time step (i.e., updating is simultaneous). The distance $R_{0}$ is assumed to be $\ll L$, the size of the flock. The direction the bird actually moves on the next time step differs from the above described direction by a random angle $\eta_{i}(t)$, with zero mean and standard deviation $\Delta$. The distribution of $\eta_{i}(t)$ is identical for all birds, time independent, and uncorrelated between different birds and different time steps. Each bird then, on the next time step, moves in the direction so chosen a distance $v_{0} \Delta t$, where the speed $v_{0}$ is the same for all birds.

To summarize, the rule for bird motion is:

$$
\theta_{i}(t+1)=\left\langle\theta_{j}(t)\right\rangle_{n}+\eta_{i}(t),
$$




$$
\begin{aligned}
& \vec{r}_{i}(t+1)=\vec{r}_{i}(t)+v_{0}(\cos \theta(t+1), \sin \theta(t+1)), \\
& \left\langle\eta_{i}(t)\right\rangle=0, \\
& \left\langle\eta_{i}(t) \eta_{j}\left(t^{\prime}\right)\right\rangle=\Delta \delta_{i j} \delta_{t t^{\prime}},
\end{aligned}
$$

where the symbol \langle\rangle$_{n}$ denotes an average over "neighbors," which are defined as the set of birds $j$ satisfying

$$
\left|\vec{r}_{j}(t)-\vec{r}_{i}(t)\right|<R_{0},
$$

\langle\rangle without the subscript $n$ denote averages over the random distribution of the noises $\eta_{i}(t)$, and $\theta_{i}(t)$ is the angle of the direction of motion of the $i$ th bird (relative to some fixed reference axis) on the time step that ends at $t$.

The flock evolves through the iteration of this rule. Note that the "neighbors" of a given bird may change on each time step, since birds do not, in general, move in exactly the same direction as their neighbors.

As first noted by Vicsek himself, this model is exactly a simple, relaxational dynamical model for an equilibrium ferromagnet, except for the motion. That is, if we interpret the $\vec{v}_{i}$ 's as "spins" carried by each bird, and update them according to the above rule, but do not actually move the birds (i.e., just treat the $\vec{v}_{i}$ 's as "pointers" carried by each bird), then the model is easily shown to be an equilibrium ferromagnet, which will relax to the Boltzmann distribution for an equilibrium Heisenberg model (albeit with the "spins" living not on a periodic lattice, as they usually do in most models and in real ferromagnets, but, rather, on a random set of points).

What Vicsek found in simulating this model largely supports the ferromagnetic analogy, with one important exception. Specifically, Vicsek found that a coherently moving, ferromagnetic flock was, indeed, possible in a system with full rotation invariance, short-ranged interaction, and "nonzero temperature" (i.e., randomness, characterized by $\Delta \neq 0$ ). This was demonstrated by the existence of a nonzero average velocity $\langle\vec{v}\rangle \equiv \frac{\sum_{i} \vec{v}_{i}}{N}$ for the entire flock for a range of values of $\Delta<\Delta_{c}$, where the critical $\Delta_{c}$ above which this order disappears is $>0$. A schematic plot of the behavior of the average velocity $|\langle\vec{v}\rangle|$ as a function of the $\Delta$ is shown in Fig. 1.

As noted by Vicsek, this looks very much like a plot of magnetization versus temperature in a ferromagnet, with $\langle\vec{v}\rangle$ playing the role of the magnetization $\vec{M} \equiv\langle\vec{S}\rangle$, and $\Delta$ playing the role of temperature, exactly as the flock-ferromagnet analogy described earlier would predict.

There is only one problem: for a two-dimensional ferromagnet, a plot like Fig. 1 would never happen. The reason for this is the well-known "Mermin-WagnerHohenberg Theorem" [12] of equilibrium statistical mechanics. This theorem states that in a thermal equilibrium model at nonzero temperature with short-ranged interactions, it is impossible to spontaneously break a continuous symmetry. This implies in particular that the equilibrium or "pointer" version of Vicsek's algorithm described above, in which the birds carry a vector $\vec{v}_{i}$ whose direction is updated according to Vicsek's algorithm, but in which the birds do not actually move, can never 


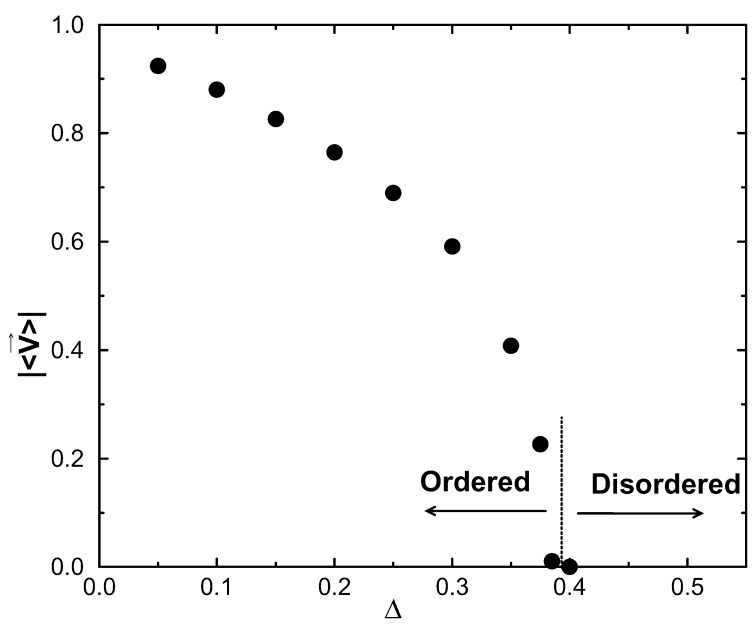

Fig. 1. The magnitude of the average velocity $|\langle\vec{v}\rangle|$ versus the noise strength $\Delta$. The existence of the two phases, the moving (ordered) phase with $|\langle\vec{v}\rangle|>0$ and the nonmoving (disordered) phase with $|\langle\vec{v}\rangle|=0$, are evident from the figure.

develop a true long-range ordered state in which all the $\vec{v}_{i}$ 's point, on average, in the same direction (more precisely, in which $\langle\vec{v}\rangle \equiv \frac{\sum_{i} \vec{v}_{i}}{N} \neq \overrightarrow{0}$ ), since such a state breaks a continuous symmetry, namely rotation invariance.

Yet the moving flock evidently has no difficulty in doing so; as Vicsek's simulation shows, even two-dimensional flocks with rotationally invariant dynamics, short-ranged interactions, and noise-i.e., seemingly all of the ingredients of the MerminWagner theorem - do move with a nonzero macroscopic velocity, which requires $\langle\vec{v}\rangle \neq \overrightarrow{0}$, which, in turn, breaks rotation invariance, in seeming violation of the theorem.

How can this be? Is the Mermin-Wagner theorem wrong? Are birds smarter than nerds?

The answer to the last two questions is, of course, no. The reason is that one of the essential premises of the Mermin-Wagner theorem does not apply to flocks: they are not systems in thermal equilibrium. The nonequilibrium aspect arises from the motion of the birds.

Clearly, it must: as described above, the motion is the only difference between Vicsek's algorithm and a (slightly unconventional) equilibrium spin system. But how does motion get around the Mermin-Wagner theorem? And, more generally, how best to understand the large-scale, long-time dynamics of a very large, moving flock?

The answer to this second question can be found in the field of hydrodynamics.

Hydrodynamics is a well-understood subject. This understanding does not come from solving the many (very many!) body problem of computing the time-dependent positions $\vec{r}_{i}(t)$ of the $10^{23}$ constituent molecules of a fluid subject to intermolecular forces from all of the other $10^{23}$ molecules. Such an approach is analytically intractable even if one knew what the intermolecular forces were. Trying to 
compute analytically the behavior of, e.g., Vicsek's algorithm directly would be the corresponding, and equally impossible, approach to the flocking problem.

Instead, the way we understand fluid mechanics is by writing down a set of continuum equations - the Navier-Stokes equations-for a continuous, smoothly varying number density $\rho(\vec{r}, t)$ and velocity $\vec{v}(\vec{r}, t)$ fields describing the fluid.

Although we know that fluids are made out of atoms and molecules, we can define "coarse-grained" number density $\rho(\vec{r}, t)$ and velocity $\vec{v}(\vec{r}, t)$ fields by averaging over "coarse-graining" volumes large compared to the intermolecular or, in the flocks, "interbird" spacing. On a large scale, even discrete systems look continuous, as we all know from close inspection of newspaper photographs and television images.

In writing down the Navier-Stokes equations, one "buries one's ignorance" [13] of the detailed microscopic dynamics of the fluid in a few phenomenological parameters, namely the mean density $\rho_{0}$, the bulk and shear viscosities $\eta_{\mathrm{B}}$ and $\eta_{\mathrm{S}}$, the thermal conductivity $\kappa$, the specific heat $c_{v}$, and the compressibility $\chi$. Once these have been deduced from experiment (or, occasionally, and at the cost of immense effort, calculated from a microscopic model), one can then predict the outcomes of all experiments that probe length scales much greater than a spatial coarse-graining scale $\ell_{0}$ and timescales $\gg t_{0}$, a corresponding microscopic time, by solving these continuum equations, a far simpler task than solving the microscopic dynamics.

But how do we write down these continuum equations? The answer to this question is, in a way, extremely simple: we write down every relevant term that is not ruled out by the symmetries and conservation laws of the problem. In the case of the Navier-Stokes equations, the symmetries are rotational invariance, space and time translation invariance, and Galilean invariance (i.e., invariance under a boost to a reference frame moving at a constant velocity), while the conservation laws are conservation of particle number, momentum, and energy.

"Relevant," in this specification means terms that are important at large-length scales and long timescales. In practice, this means a "gradient expansion": we only keep in the equations of motion terms with the smallest possible number of space and time derivatives. For example, in the Navier-Stokes equations we keep a viscous term $\eta_{s} \nabla^{2} \vec{v}$, but not a term $\gamma \nabla^{4} \vec{v}$, though the latter is also allowed by symmetry, because the $\gamma \nabla^{4} \vec{v}$ term involves more spatial derivatives, and hence is smaller, for slow spatial variation, than the viscous term we have already got.

In Section 2, we will review the formulation and solution of such a hydrodynamic model for "ferromagnetic" flocks in [14-16].

In addition to these symmetries of the questions of motion, which reflect the underlying symmetries of the physical situation under consideration, it is also necessary to treat correctly the symmetries of the state of the system under consideration. These may be different from those of the underlying system, precisely because the system may spontaneously break one or more of the underlying symmetries of the equations of motion. Indeed, this is precisely what happens in the ordered state of a ferromagnet: the underlying rotation invariance of the system as a whole is broken by the system in its steady state, in which a unique direction is picked out - namely, the direction of the spontaneous magnetization. 
As should be apparent from our earlier discussion, this is also what happens in a spontaneously moving flock. Indeed, the symmetry that is broken - rotational - and the manner in which it is broken - namely, the development of a nonzero expectation value for some vector (the spin $\vec{S}$ in the ferromagnetic case; the velocity $\langle\vec{v}\rangle$ in the flock) are precisely the same in both cases [7].

Many different "phases" [17], in this sense of the word, of a system with a given underlying symmetry are possible. Indeed, we have already described two such phases of flocks: the "ferromagnetic" or moving flock, and the "disordered," "paramagnetic," or stationary flock.

In equilibrium statistical mechanics, this is precisely how we classify different phases of matter: by the underlying symmetries that they break. Crystalline solids, for example, differ from fluids (liquid and gases) by breaking both translational and orientational symmetry. Less familiar to those outside the discipline of soft condensed matter physics are the host of mesophases known as liquid crystals, in some of which (e.g., nematics [18]) only orientational symmetry is broken, while in others, (e.g., smectics [18]) translational symmetry is only broken in some directions, not all.

It seems clear that, at least in principle, every phase known in condensed matter systems could also be found in flocks. To date, hydrodynamic models have been formulated for three such phases: the paramagnetic and ferromagnetic state [14 $16,19,20]$ and the nematic [21] state. In intriguing contrast to the situation in thermal equilibrium systems, the long-wavelength stability of such phases is found to depend on the type of dynamics (momentum-conserving versus nonconserving, inertial versus viscosity-dominated) obeyed by the system [19,21].

In particular, the theoretical work on the ferromagnetic state explains how such systems "get around" the Mermin-Wagner theorem, and exhibit long-ranged order even in $d=2$.

In Section 5, we summarize the theoretical work on what we call "nematic" flocks, which are flocks in which the motion and/or orientation of the creatures picks out an axis, but not a sense along that axis. This could happen (for example, but not exclusively) if the system settled down into a state in which creatures moved preferentially along the $x$ axis, say, half in the $+x$ and half in the $-x$ direction with the $+x$ and $-x$ creatures well mixed. The state would then have zero mean velocity for the flock, but would be uniaxially ordered. Nematic phases have been observed in, e.g., living melanocytes [9]. Dynamical states of exactly the same symmetry occur in agitated granular materials composed of long, thin grains [22-24]. Surprisingly, even though such flocks have no net motion, the continuum theory for this state, developed along the same lines as that for the ferromagnetic state, predicts that their behavior is very different from that of conventional equilibrium nematic liquid crystals, despite the fact that they have the same symmetry, just as ferromagnetic flocks are quite different from their equilibrium counterparts.

In addition to changing which symmetries are broken (i.e., which phase we are considering) in a flock, we can also consider different underlying symmetries. The simplest such change of underlying symmetry is considered in Section 4, in which we treat ferromagnetic flocks which move in a non-rotationally invariant environ- 
ment. Specifically, we consider "easy-plane" models, in which the "birds" prefer to fly in a particular plane (e.g., horizontally), which is obviously the case for many real examples.

A more dramatic change is to restore Galilean invariance. The work on ferromagnetic and nematic flocks described above dealt with systems lacked this symmetry, which is usually included in fluid mechanics. To lack Galilean invariance simply means that the equations of motion do not remain the same in a moving coordinate system. This is appropriate if we are modeling creatures moving in the presence of friction over (or through) a static medium; e.g., wildebeest moving over the surface of the Serengeti plane, bacteria crawling over the surface of a Petri dish, etc. It is equally clearly not appropriate for creatures moving through a medium which is itself fluid (e.g., the air birds fly through, the water fish swim through). In these cases, there is an additional symmetry (Galilean invariance) not present in the previous models, which leads to additional conservation laws (of total momentum of flock plus background fluid), which in turn lead to additional hydrodynamic variables (e.g., total momentum density), and a completely different hydrodynamic description.

Hydrodynamic models of both ferromagnetic and nematic flocks moving through such a background fluid [19] are reviewed in Section 6, where it is shown that nematic flocks in suspension have an inviscid instability at long wavelengths. The most striking prediction of this section, however, is that "ferromagnetic" flocks in suspension are unstable at sufficiently low Reynolds number. This means it is impossible in principle to find long-range ordered swimming bacteria; in the absence of external aligning fields, a large flock of bacteria initially all swimming in the same direction must break up into finite flocks with velocities uncorrelated from flock to flock. The section also summarizes predictions [20] for novel rheological properties of isotropic flocks as their correlation length and time are increased. Experimental evidence for the instability of ferromagnetic flocks in suspension is reviewed in Section 7, along with many other experiments done on flocking.

In Section 8, we discuss a number of experiments we would like to see done. Theory is currently far ahead of experiment in this field, an unhealthy situation that can be corrected by careful measurements of fluctuations in flocks to test, quantitatively, the many detailed predictions that are available from the theories described in Sections $2-5$.

Finally, in Section 9, we discuss possible directions for future research in this area. We hope to make clear there that the subject of flocking is an extremely rich and fertile one, the surface of which we have scarcely scratched. In particular, as discussed earlier, virtually every one of the dozens of phases known in soft condensed matter physics should have an analog in flocks. So far, as mentioned earlier, only three of these phases have even had their hydrodynamics formulated, and only two of them (the ordered ferromagnetic and nematic states) have really been investigated thoroughly. The study of the rich variety of other possible phases of flocks therefore remains a wide open subject, potentially as intriguing as any in Condensed Matter of Physics, as well as being of obvious interest to anyone interested in biology, zoology, 
and dynamical systems. We hope that this review will stimulate further research on this rich, fascinating, and still largely unexplored subject.

The remainder of this paper is organized as follows: in Section 2, we review the hydrodynamic theory of ferromagnetic flocks in isotropic (i.e, fully rotationally invariant) environments. In Section 3, we describe numerical experiments confirming the theory in detail, and addressing the phase transitions in these systems as well. In Section 4, we consider "easy-plane" models, in which the "birds" prefer to fly in a particular plane (e.g., horizontally), which is obviously the case of many real examples. In these models, the positions of the birds are fully extended over 3 (or, as a theorist's toy model, more) dimensions; it is just the velocities of the birds that lie preferentially in a plane (e.g., consider a tall, broad, and deep flock of flamingoes flying horizontally). In Section 5, we discuss nematic flocking, while Section 6 treats the incorporation of "solvent hydrodynamic effects" (e.g., the motion of the background fluid) on ferromagnetic, nematic, and disordered flocks. In Section 7, we discuss the experimental work that has been done to date testing some of these ideas, while in Section 8 we provide a "wish-list" of experiments we would like to see done, which would provide detailed quantitative tests of the theories we describe here. This section will also lay out in detail precisely what those quantitative predictions are, and how experiments can test them. Experimentalists interested in testing our ideas should proceed directly to this section, which is fairly self-contained.

And finally, we conclude in Section 9 by suggesting several directions for future work. Our list is necessarily abbreviated; any clever reader can no doubt think of many equally fascinating problems in this area which are not on our list, but should be studied. This is a fascinating field with room for many more researchers, both theoretical and experimental.

\section{Isotropic ferromagnetic flocks}

\subsection{Formulating the hydrodynamic model}

In this section, we will review the derivation and analysis of the hydrodynamic model of ferromagnetic flocks. A more detailed discussion can be found in [16]. As discussed in Section 1, the system we wish to model is any collection of a large number $N$ of organisms (hereafter referred to as "birds") in a $d$-dimensional space, with each organism seeking to move in the same direction as its immediate neighbors.

We further assume that each organism has no "compass"; in the sense defined in Section 1, i.e., no intrinsically preferred direction in which it wishes to move. Rather, it is equally happy to move in any direction picked by its neighbors. However, the navigation of each organism is not perfect; it makes some errors in attempting to follow its neighbors. We consider the case in which these errors have zero mean; e.g., in two dimensions, a given bird is no more likely to err to the right than to the left of the direction picked by its neighbors. We also assume that these errors have no long temporal correlations; e.g., a bird that has erred to the right at time $t$ is equally likely to err either left or right at a time $t^{\prime}$ much later than $t$. 
The continuum model will describe the long distance behavior of any flock satisfying the symmetry conditions we shall specify in a moment. The automaton studied by Vicsek et al. [5] described in Section 1 provides one concrete realization of such a model. Adding "bells and whistles" to this model by, e.g., including purely attractive or repulsive interactions between the birds, restricting their field of vision to those birds ahead of them, giving them some short-term memory, etc., will not change the hydrodynamic model, but can be incorporated simply into a change of the numerical values of a few phenomenological parameters in the model, in much the same way that all simple fluids are described by the Navier-Stokes equations, and changing fluids can be accounted for simply by changing, e.g., the viscosity that appears in those equations.

This model should also describe real flocks of real living organisms, provided that the flocks are large enough, and that they have the same symmetries and conservation laws that, e.g., Vicsek's algorithm does.

So, given this lengthy preamble, what are the symmetries and conservation laws of flocks?

The only symmetries of the model are invariance under rotations and translations. Translation-invariance simply means that displacing the positions of the whole flock rigidly by a constant amount has no physical effect, since the space the flock moves through is assumed to be on average homogeneous [25]. Since we are not considering translational ordering, this symmetry remains unbroken and plays no interesting role in what follows, any more than it would in a fluid. Rotation invariance simply says the "birds" lack a compass, so that all direction of space are equivalent to other directions. Thus, the "hydrodynamic" equation of motion we write down cannot have built into it any special direction picked "a priori"; all directions must be spontaneously picked out by the motion and spatial structure of the flock. As we shall see, this symmetry severely restricts the allowed terms in the equation of motion.

Note that the model does not have Galilean invariance: changing the velocities of all the birds by some constant boost $\vec{v}_{b}$ does not leave the model invariant. Indeed, such a boost is impossible in a model that strictly obeys Vicsek's rules, since the speeds of all the birds will not remain equal to $v_{0}$ after the boost. One could image relaxing this constraint on the speed, and allowing birds to occasionally speed up or slow down, while tending an average to move at speed $v_{0}$. Then the boost just described would be possible, but clearly would change the subsequent evolution of the flock.

Another way to say this is that birds move through a resistive medium, which provides a special Galilean reference frame, in which the dynamics are particularly simple, and different from those in other reference frames. Since real organisms in flocks always move through such a medium (birds through the air, fish through the sea, wildebeest through the arid dust of the Serengeti), this is a very realistic feature of the model [26].

As we shall see shortly, this lack of Galilean invariance allows terms in the hydrodynamic equations of birds that are not present in, e.g., the Navier-Stokes equations for a simple fluid, which must be Galilean invariant, due to the absence of a luminiferous ether. 
The sole conservation law for flocks is conservation of birds: we do not allow birds to be born or die "on the wing."

In contrast to the Navier-Stokes equation, there is no conservation of momentum in the models discussed in this section. This is, ultimately, a consequence of the absence of Galilean invariance.

Having established the symmetries and conservation laws constraining our model, we need now to identify the hydrodynamic variables. They are precisely the same as those of a simple fluid [27]: the coarse grained bird velocity field $\vec{v}(\vec{r}, t)$, and the coarse grained bird density $\rho(\vec{r}, t)$. The field $\vec{v}(\vec{r}, t)$, which is defined for all $\vec{r}$, is a suitable weighted average of the velocities of the individual birds in some volume centered on $\vec{r}$. This volume is big enough to contain enough birds to make the average well-behaved, but should have a spatial linear extent of no more than a few "microscopic" lengths (i.e., the interbird distance, or by a few times the interaction range $\left.R_{0}\right)$. By suitable weighting, we seek to make $\vec{v}(\vec{r}, t)$ fairly smoothly varying in space.

The density $\rho(\vec{r}, t)$ is similarly defined, being just the number of particles in a coarse graining volume, divided by that volume.

The exact prescription for the coarse graining should be unimportant, so long as $\rho(\vec{r}, t)$ is normalized so as to obey the "sum rule" that its integral over any macroscopic volume (i.e., any volume compared with the aforementioned microscopic lengths) be the total number of birds in that volume. Indeed, the coarse graining description just outlined is the way that one imagines, in principle, going over from a description of a simple fluid in terms of equations of motion for the individual constituent molecules to the continuum description of the Navier-Stokes equation.

We will also follow the historical precedent of the Navier-Stokes $[13,28]$ equation by deriving our continuum, long wavelength description of the flock not by explicitly coarse graining the microscopic dynamics (a very difficult procedure in practice), but, rather, by writing down the most general continuum equations of motion for $\vec{v}$ and $\rho$ consistent with the symmetries and conservation laws of the problem. This approach allows us to bury our ignorance in a few phenomenological parameters, (e.g., the viscosity in the Navier-Stokes equation) whose numerical values will depend on the detailed microscopic rules of individual bird motion. What terms can be present in the EOMs, however, should depend only on symmetries and conservation laws, and not on other aspects of the microscopic rules.

To reduce the complexity of our equations of motion still further, we will perform a spatio-temporal gradient expansion, and keep only the lowest order terms in gradients and time derivatives of $\vec{v}$ and $\rho$. This is motivated and justified by our desire to consider only the long distance, long time properties of the flock. Higher order terms in the gradient expansion are "irrelevant": they can lead to finite "renormalization" of the phenomenological parameters of the long wavelength theory, but cannot change the type of scaling of the allowed terms.

With this lengthy preamble in mind, we now write down the equations of motion:

$$
\begin{aligned}
\partial_{t} \vec{v} & +\lambda_{1}(\vec{v} \cdot \vec{\nabla}) \vec{v}+\lambda_{2}(\vec{\nabla} \cdot \vec{v}) \vec{v}+\lambda_{3} \vec{\nabla}\left(|\vec{v}|^{2}\right) \\
& =\alpha \vec{v}-\beta|\vec{v}|^{2} \vec{v}-\overrightarrow{\nabla P}+D_{\mathrm{B}} \vec{\nabla}(\vec{\nabla} \cdot \vec{v})+D_{\mathrm{T}} \nabla^{2} \vec{v}+D_{2}(\vec{v} \cdot \vec{\nabla})^{2} \vec{v}+\vec{f},
\end{aligned}
$$




$$
\begin{aligned}
& P=P(\rho)=\sum_{n=1}^{\infty} \sigma_{n}\left(\rho-\rho_{0}\right)^{n}, \\
& \frac{\partial \rho}{\partial t}+\nabla \cdot(\vec{v} \rho)=0,
\end{aligned}
$$

where $\beta, D_{\mathrm{B}}, D_{2}$, and $D_{\mathrm{T}}$ are all positive, and $\alpha<0$ in the disordered phase and $\alpha>0$ in the ordered state (in mean field theory). The origin of the various terms is as follows: the $\lambda$ terms on the left-hand side of Eq. (6) are the analogs of the usual convective derivative of the coarse-grained velocity field $\vec{v}$ in the Navier-Stokes equation. Here the absence of Galilean invariance allows all three combinations of one spatial gradient and two velocities that transform like vectors; if Galilean invariance did apply here, it would force $\lambda_{2}=\lambda_{3}=0$ and $\lambda_{1}=1$. However, as we have argued above, we are not constrained here by Galilean invariance, and so all three coefficients are nonzero phenomenological parameters whose nonuniversal values are determined by the microscopic rules. The $\alpha$ and $\beta$ terms simply make the local $\vec{v}$ have a nonzero magnitude $(=\sqrt{\alpha / \beta})$ in the ordered phase, where $\alpha>0 . D_{\mathrm{B}, \mathrm{T}, 2}$ are the diffusion constants (or viscosities) reflecting the tendency of a localized fluctuation in the velocities to spread out because of the coupling between neighboring "birds." Amusingly, it is these "viscous" terms that contain the "elasticity" of an ordered flock-the restoring torques that try to make parallel the orientation of neighboring birds. The $\vec{f}$ term is a random driving force representing the noise. We assume it is Gaussian with white noise correlations

$$
\left\langle f_{i}(\vec{r}, t) f_{j}\left(\vec{r}^{\prime}, t^{\prime}\right)\right\rangle=\Delta \delta_{i j} \delta^{d}\left(\vec{r}-\vec{r}^{\prime}\right) \delta\left(t-t^{\prime}\right),
$$

where $\Delta$ is a constant, and $i, j$ denote Cartesian components. Finally, $P$ is the pressure, which tends to maintain the local number density $\rho(\vec{r})$ at its mean value $\rho_{0}$, and $\delta \rho=\rho-\rho_{0}$. Strictly speaking, here too, as in the case of the "viscous" terms involving $D_{\mathrm{B}, \mathrm{T}, 2}$, we should distinguish gradients parallel and perpendicular to $\vec{v}$, i.e., gradients in the density should be allowed to have independent effects along and transverse to $\vec{v}$ in (6). In an equilibrium fluid this could not happen, since Pascal's Law ensures that pressure is isotropic. In the nonequilibrium steady state of a flock, no such constraint applies. For simplicity, however, we ignore this possibility here, and consider purely longitudinal pressure forces.

The final Eq. (8) is just conservation of bird number (we do not allow our birds to reproduce or die on the wing).

Symmetry allows any of the phenomenological coefficients $\lambda_{i}, \alpha, \sigma_{n}, \beta$, and $D_{i}$ in Eqs. (6) and (7) to be functions of the squared magnitude $|\vec{v}|^{2}$ of the velocity, and of the density $\rho$ as well.

\subsection{The broken symmetry ferromagnetic state}

The hydrodynamic model embodied in Eqs. (6)-(8) is equally valid in both the "disordered" (i.e., nonmoving) $(\alpha<0)$ and "ferromagnetically ordered" (i.e., moving) $(\alpha<0)$ state. In this section, we are mainly interested in the 'ferromagnetically 
ordered," broken-symmetry phase; specifically in whether fluctuations around the symmetry broken ground state destroy it (as in the analogous phase of the 2D XY model). For $\alpha>0$, we can write the velocity field as $\vec{v}=v_{0} \hat{x}_{\|}+\overrightarrow{\delta v}$, where $v_{0} \hat{x}_{\|}=\langle\vec{v}\rangle$ is the spontaneous average value of $\vec{v}$ in the ordered phase. We will chose $v_{0}=\frac{\sqrt{\alpha}}{\beta}$ (which should be thought of as an implicit condition on $v_{0}$, since $\alpha$ and $\beta$ can, in general, depend on $|\vec{v}|^{2}$ ); with this choice, the equation of motion for the fluctuation $\delta v_{\|}$of $v_{\|}$is

$$
\partial_{t} \delta v_{\|}=-\sigma_{1} \partial_{\|} \delta \rho-2 \alpha \delta v_{\|}+\text {irrelevant terms. }
$$

Note now that if we are interested in "hydrodynamic" modes, by which we mean modes for which frequency $\omega \rightarrow 0$ as wave vector $q \rightarrow 0$, we can, in the hydrodynamic $(\omega, q \rightarrow 0)$ limit, neglect $\partial_{t} \delta v_{\|}$relative to $\alpha \delta v_{\|}$in (10). The resultant equation can trivially be solved for $\delta v_{\|}$

$$
\delta v_{\|}=-\left(\sigma_{1} / 2 \alpha\right) \partial_{\|} \delta \rho .
$$

Inserting (11) in the equations of motion for $\vec{v}_{\perp}$ and $\delta \rho$, we obtain, neglecting "irrelevant" terms:

$$
\begin{aligned}
& \partial_{t} \vec{v}_{\perp}+\gamma \partial_{\|} \vec{v}_{\perp}+\lambda_{1}\left(\vec{v}_{\perp} \cdot \vec{\nabla}_{\perp}\right) \vec{v}_{\perp}+\lambda_{2}\left(\vec{\nabla}_{\perp} \cdot \vec{v}_{\perp}\right) \vec{v}_{\perp} \\
& \quad=-\vec{\nabla}_{\perp} P+D_{\mathrm{B}} \vec{\nabla}_{\perp}\left(\vec{\nabla}_{\perp} \cdot \vec{v}_{\perp}\right)+D_{\mathrm{T}} \nabla_{\perp}^{2} \vec{v}_{\perp}+D_{\|} \partial_{\|}^{2} \vec{v}_{\perp}+\vec{f}_{\perp}, \\
& \frac{\partial \delta \rho}{\partial t}+\rho_{\mathrm{o}} \vec{\nabla}_{\perp} \cdot \vec{v}_{\perp}+\vec{\nabla}_{\perp} \cdot\left(\vec{v}_{\perp} \delta \rho\right)+v_{0} \partial_{\|} \delta \rho=D_{\rho} \partial_{\|}^{2} \delta \rho,
\end{aligned}
$$

where $D_{\rho} \equiv \rho_{0} \frac{\sigma_{1}}{2 \alpha}, D_{\mathrm{B}}, D_{\mathrm{T}}$, and $D_{\|} \equiv D_{\mathrm{T}}+D_{2} v_{0}^{2}$ are the diffusion constants, and we have defined

$$
\gamma \equiv \lambda_{1} v_{0}
$$

The pressure $P$ continues to be given, as it always will, by Eq. (7).

From this point forward, we will treat the phenomenological parameters $\lambda_{i}, \gamma$, and $D_{i}$ appearing in Eqs. (12) and (13) as constants, since they depend, in our original model (6), only on the scalar quantities $|\vec{v}|^{2}$ and $\rho(\vec{r})$, whose fluctuations in the broken symmetry state away from their mean values $v_{0}^{2}$ and $\rho_{0}$ are small. Furthermore, these fluctuations lead only to "irrelevant" terms in the equations of motion.

It should be emphasized here that, once nonlinear fluctuation effects are included, the $v_{0}$ in Eq. (13) will not be given by the "mean" velocity of the birds, in the sense of

$$
\langle v\rangle \equiv \frac{\left|\sum_{i} \vec{v}_{i}\right|}{N},
$$

where $N$ is the number of birds. This is because, in our continuum language

$$
\langle v\rangle=\frac{\left|\left\langle\int \rho(\vec{r}, t) \vec{v}(\vec{r}, t) \mathrm{d}^{d} r\right\rangle\right|}{\left\langle\int \rho(\vec{r}, t) \mathrm{d}^{d} r\right\rangle}=\frac{|\langle\rho \vec{v}\rangle|}{\langle\rho\rangle}
$$

while $v_{0}$ in Eq. (14) is

$$
v_{0}=|\langle\vec{v}(\vec{r}, t)\rangle| \text {. }
$$


Once $\rho$ fluctuates, so that $\rho=\langle\rho\rangle+\delta \rho$, the "mean" velocity of the birds

$$
\langle v\rangle=\left|\frac{\langle\rho \vec{v}\rangle}{\langle\rho\rangle}\right|=\left|\frac{\langle\rho\rangle\langle\vec{v}\rangle}{\langle\rho\rangle}+\frac{\langle\delta \rho \vec{v}\rangle}{\langle\rho\rangle}\right|
$$

which equals $v_{0} \equiv|\langle\vec{v}\rangle|$ only if the correlation function $\langle\delta \rho \vec{v}\rangle=0$, which it will not, in general. For instance, one could easily imagine that denser regions of the flock might move faster; in which case $\langle\delta \rho \vec{v}\rangle$ would be positive along $\langle\vec{v}\rangle$. Thus, $\langle\vec{v}\rangle$ measured in a simulation by simply averaging the speed of all birds, as in Eq. (15), will not be equal to $v_{0}$ in Eq. (14). Indeed, we can think of no simple way to measure $v_{0}$, and so chose instead to think of it as an additional phenomenological parameter in the broken symmetry state equations of motion (12) and (13). It should, in simulations and experiments, be determined by fitting the correlation functions we will calculate in the next section. One should not expect it to be given by $\langle v\rangle$ as defined in Eq. (16).

Similar considerations apply to $\gamma$ : it should also be thought of as an independent, phenomenological parameter, not necessarily determined by the mean velocity and nonlinear parameter $\lambda_{1}$ through (14).

\subsection{Linearized theory of the broken symmetry ferromagnetic state}

As a first step towards understanding the implications of these equations of motion, we linearize them in $\vec{v}_{\perp}$ and $\delta \rho \equiv \rho-\rho_{0}$. Doing this, and Fourier transforming in space and time, we obtain the linear equations:

$$
\begin{aligned}
& {\left[-\mathrm{i}\left(\omega-\gamma q_{\|}\right)+\Gamma_{\mathrm{T}}(\vec{q})\right] \vec{v}_{\mathrm{T}}(\vec{q}, \omega)=\vec{f}_{\mathrm{T}}(\vec{q}, \omega),} \\
& {\left[-\mathrm{i}\left(\omega-\gamma q_{\|}\right)+\Gamma_{\mathrm{L}}(\vec{q})\right] v_{\mathrm{L}}+\mathrm{i} \sigma_{1} q_{\perp} \delta \rho=f_{\mathrm{L}}(\vec{q}, \omega),} \\
& {\left[-\mathrm{i}\left(\omega-v_{0} q_{\|}\right)+\Gamma_{\rho}(\vec{q})\right] \delta \rho+\mathrm{i} \rho_{0} q_{\perp} v_{\mathrm{L}}=0,}
\end{aligned}
$$

where

and

$$
v_{\mathrm{L}}(\vec{q}, \omega) \equiv \frac{\vec{q}_{\perp} \cdot \vec{v}_{\perp}(\vec{q}, \omega)}{q_{\perp}}
$$

$$
\vec{v}_{\mathrm{T}}(\vec{q}, \omega)=\vec{v}_{\perp}(\vec{q}, \omega)-\frac{\vec{q}_{\perp} v_{\mathrm{L}}}{q_{\perp}}
$$

are the longitudinal and transverse (to $\vec{q}_{\perp}$ ) pieces of the velocity, $\vec{f}_{\mathrm{T}}(\vec{q}, \omega)$ and $f_{\mathrm{L}}(\vec{q}, \omega)$ are the analogous pieces of the Fourier transformed random force $\vec{f}(\vec{q}, \omega)$, and we have defined wavevector dependent transverse, longitudinal, and $\rho$ dampings $\Gamma_{\mathrm{L}, \mathrm{T}, \rho}$ :

$$
\begin{aligned}
& \Gamma_{\mathrm{L}}(\vec{q}) \equiv D_{\mathrm{L}} q_{\perp}^{2}+D_{\|} q_{\|}^{2}, \\
& \Gamma_{\mathrm{T}}(\vec{q})=D_{\mathrm{T}} q_{\perp}^{2}+D_{\|} q_{\|}^{2}, \\
& \Gamma_{\rho}(\vec{q})=D_{\rho} q_{\|}^{2},
\end{aligned}
$$

where we have defined $D_{\mathrm{L}} \equiv D_{\mathrm{T}}+D_{\mathrm{B}}, q_{\perp}=\left|\vec{q}_{\perp}\right|$. 
Note that in $d=2$, the transverse velocity $\vec{v}_{\mathrm{T}}$ does not exist: no vector can be perpendicular to both the $x_{\|}$axis and $\vec{q}_{\perp}$ in two dimensions. This leads to many important simplifications in $d=2$, as we will see later; these simplifications make it (barely) possible to get exact exponents in $d=2$ for the full, nonlinear problem.

It is now a straightforward exercise in linear algebra to solve these linearized equations for the hydrodynamic mode structure of a flock. By "hydrodynamic mode-structure" we simply mean the eigenfrequencies $\omega(\vec{q})$ of the homogeneous equations obtained by setting the noise term $\vec{f}=\overrightarrow{0}$. It is just as straightforward to solve these linearized equations for $\vec{v}(\vec{q}, \omega)$ and $\rho(\vec{q}, \omega)$ in terms of $\vec{f}(\vec{q}, \omega)$. Using the known correlations of $\vec{f}$ from Eq. (9) given earlier, one can thereby straightforwardly compute the correlations of $\vec{v}(\vec{q}, \omega)$ and $\rho(\vec{q}, \omega)$ with each other, and with themselves. Readers interested in the details of these calculations are referred to [16]; here we simply summarize the results.

The normal modes of these equations are $d-2$ purely diffusive transverse modes associated with $\vec{v}_{\mathrm{T}}$, all of which have the same eigenfrequency

$$
\omega_{\mathrm{T}}=\gamma q_{\|}-\mathrm{i} \Gamma_{\mathrm{T}}(\vec{q})=\gamma q_{\|}-\mathrm{i}\left(D_{\mathrm{T}} q_{\perp}^{2}+D_{\|} q_{\|}^{2}\right)
$$

and a pair of damped, propagating sound modes with complex (in both senses of the word) eigenfrequencies

$$
\begin{aligned}
\omega_{ \pm} & =c_{ \pm}\left(\theta_{\vec{q}}\right) q-\mathrm{i} \Gamma_{\mathrm{L}}\left[\frac{v_{ \pm}\left(\theta_{\vec{q}}\right)}{2 c_{2}\left(\theta_{\vec{q}}\right)}\right]-\mathrm{i} \Gamma_{\rho}\left[\frac{v_{\mp}\left(\theta_{\vec{q}}\right)}{2 c_{2}\left(\theta_{\vec{q}}\right)}\right] \\
& =c_{ \pm}\left(\theta_{\vec{q}}\right) q-\mathrm{i}\left(D_{L} q_{\|}^{2}+D_{\perp} q_{\perp}^{2}\right)\left[\frac{v_{ \pm}\left(\theta_{\vec{q}}\right)}{2 c_{2}\left(\theta_{\vec{q}}\right)}\right]-\mathrm{i} D_{\rho} q_{\|}^{2}\left[\frac{v_{\mp}\left(\theta_{\vec{q}}\right)}{2 c_{2}\left(\theta_{\vec{q}}\right)}\right],
\end{aligned}
$$

where $\theta_{\vec{q}}$ is the angle between $\vec{q}$ and the direction of flock motion (i.e., the $x_{\|}$axis):

$$
\begin{aligned}
& c_{ \pm}\left(\theta_{\vec{q}}\right) \equiv \frac{\gamma+v_{0}}{2} \cos \left(\theta_{\vec{q}}\right) \pm c_{2}\left(\theta_{\vec{q}}\right), \\
& v_{ \pm}\left(\theta_{\vec{q}}\right) \equiv \pm \frac{\gamma-v_{0}}{2} \cos \left(\theta_{\vec{q}}\right)+c_{2}\left(\theta_{\vec{q}}\right), \\
& c_{2}\left(\theta_{\vec{q}}\right) \equiv \sqrt{\frac{1}{4}\left(\gamma-v_{0}\right)^{2} \cos ^{2}\left(\theta_{\vec{q}}\right)+c_{0}^{2} \sin ^{2}\left(\theta_{\vec{q}}\right)},
\end{aligned}
$$

and $c_{0} \equiv \sqrt{\sigma_{1} \rho_{0}}$. A polar plot of this highly anisotropic sound speed is given in Fig. 2.

We remind the reader that here and hereafter, we only keep the leading order terms in the long wave length limit, i.e., for small $q_{\|}$and $q_{\perp}$.

These direction-dependent sound speeds can most easily be determined experimentally by measuring the spatio-temporally Fourier-transformed density-density correlation function $C_{\rho \rho}(\vec{q}, \omega) \equiv\left\langle|\rho(\vec{q}, \omega)|^{2}\right\rangle$. We will describe in detail in Section 7 how to easily obtain this correlation function from observation of a flock via, e.g., computer imaging of a film. The linearized calculation described above predicts that this density-density correlation function, when considered as a function of frequency $\omega$, has two sharp peaks at $\omega=c_{ \pm}\left(\theta_{\vec{q}}\right) q$, with widths of $\mathrm{O}\left(q^{2}\right)$, as illustrated in Fig. 3 . 


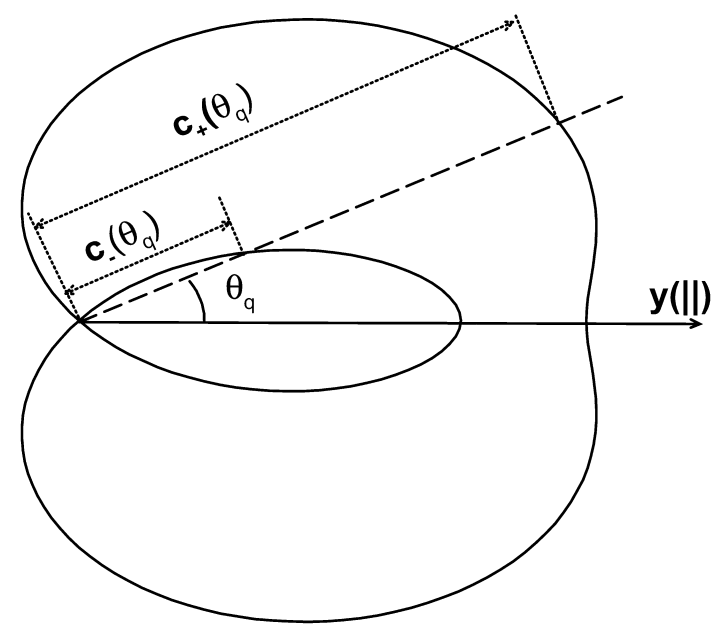

Fig. 2. Polar plot of the direction-dependent sound speeds $c_{ \pm}\left(\theta_{\vec{q}}\right)$, with the horizontal axis along the direction of mean flock motion.

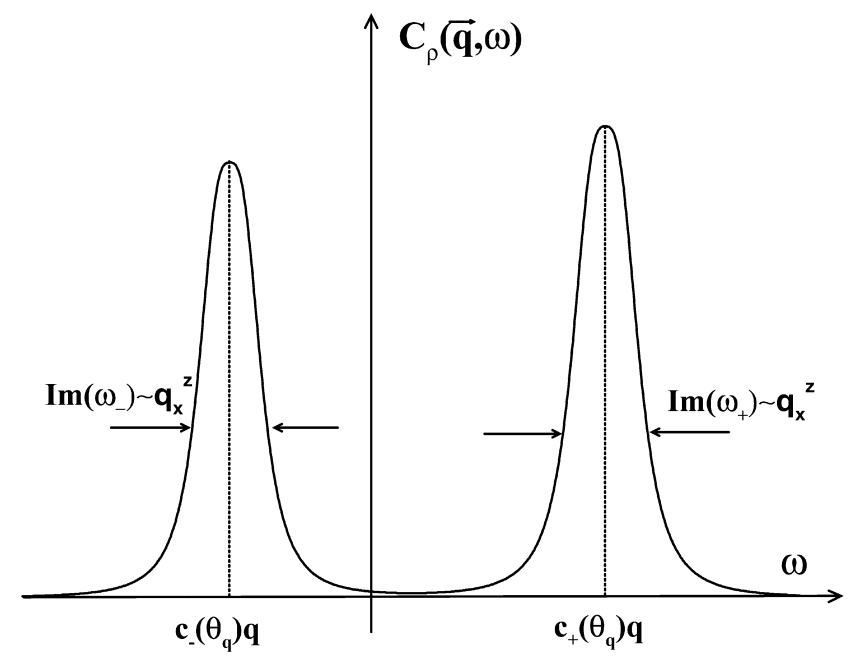

Fig. 3. Plot of the spatio-temporally Fourier-transformed density correlation function $C_{\rho}(\vec{q}, \omega)$ versus $\omega$ for fixed $\vec{q}$. It shows two sharp asymmetrical peaks at $\omega=c_{ \pm}\left(\theta_{\vec{q}}\right) q$ associated with the sound modes of the flock, where $c_{ \pm}\left(\theta_{\vec{q}}\right)$ are the sound mode speeds. The widths of those peaks are the second mode dampings $\operatorname{Im} \omega_{ \pm}\left(\theta_{\vec{q}}\right) \propto q_{\perp}^{z} f_{ \pm}\left(\frac{q_{\|} \ell_{0}}{\left(q_{\perp} \ell_{0}\right)^{\frac{1}{2}}}\right)$.

We call these peaks "sharp" precisely because their widths, for $q \rightarrow 0$, are much smaller than their displacement from the origin (of $\omega$ ). Indeed, as $q \rightarrow 0$, they become infinitely sharp in this sense.

All of the above results persist in the full, nonlinear theory we will describe later except the scaling of the widths, which becomes anisotropic and "anomalous," as 
will be described in Section 2.4. The peaks do remain sharp, however, and their positions are correctly predicted by the linearized theory.

The exact expression for $C_{\rho \rho}(\vec{q}, \omega)$ that we obtain is

$$
C_{\rho \rho}=\frac{\Delta \rho_{0}^{2} q_{\perp}^{2}}{\left(\omega-c_{+}\left(\theta_{\vec{q}}\right) q\right)^{2}\left(\omega-c_{-}\left(\theta_{\vec{q}}\right) q\right)^{2}+\left(\omega\left(\Gamma_{\mathrm{L}}(\vec{q})+\Gamma_{\rho}(\vec{q})\right)-q_{\|}\left(v_{0} \Gamma_{\mathrm{L}}(\vec{q})+\gamma \Gamma_{\rho}(\vec{q})\right)\right)^{2}} .
$$

We can similarly find the velocity autocorrelations

$$
C_{i j}(\vec{q}, \omega) \equiv\left\langle v_{i}^{\perp}(-\vec{q},-\omega) v_{j}^{\perp}(\vec{q}, \omega)\right\rangle \equiv C_{\mathrm{TT}}(\vec{q}, \omega) P_{i j}^{\perp}(\vec{q})+C_{\mathrm{LL}}(\vec{q}, \omega) L_{i j}^{\perp}(\vec{q}),
$$

where:

$$
\begin{aligned}
& L_{i j}^{\perp}(\vec{q}) \equiv \frac{q_{i}^{\perp} q_{j}^{\perp}}{q_{\perp}^{2}}, \\
& P_{i j}^{\perp}(\vec{q}) \equiv \delta_{i j}^{\perp}-L_{i j}^{\perp}(\vec{q}),
\end{aligned}
$$

are longitudinal and transverse projection operators in the plane perpendicular to the mean flock motion

$$
C_{\mathrm{TT}}(\vec{q}, \omega)=\frac{\Delta}{\left(\omega-\gamma q_{\|}\right)^{2}+\Gamma_{\mathrm{T}}^{2}(\vec{q})}
$$

and

$$
C_{\mathrm{LL}}(\vec{q}, \omega)=\frac{\Delta\left(\left(\omega-v_{0} q_{\|}\right)^{2}+\Gamma_{\rho}^{2}(\vec{q})\right)}{\left(\omega-c_{+}\left(\theta_{\vec{q}}\right) q\right)^{2}\left(\omega-c_{-}\left(\theta_{\vec{q}}\right) q\right)^{2}+\left(\omega\left(\Gamma_{\mathrm{L}}(\vec{q})+\Gamma_{\rho}(\vec{q})\right)-q_{\|}\left(v_{0} \Gamma_{\mathrm{L}}(\vec{q})+\gamma \Gamma_{\rho}(\vec{q})\right)\right)^{2}} .
$$

The transverse and longitudinal correlation functions in Eqs. (36) and (37) are plotted as functions of $\omega$ for fixed $\vec{q}$ in Fig. 4 .

Note that they have weight in entirely different regions of frequency: $C_{\mathrm{TT}}$ is peaked at $\omega=\gamma q_{\|}$, while $C_{\mathrm{LL}}$, like $C_{\rho \rho}$, has two peaks, at $\omega=c_{ \pm}\left(\theta_{\vec{q}}\right) q$. Since all three peaks have widths of order $q^{2}$, there is little overlap between the transverse and the longitudinal peaks as $|\vec{q}| \rightarrow 0$.

With the velocity correlations $C_{i j}(\vec{q}, \omega)$ in hand, we can now address the question which first caught our attention: Are birds smarter than nerds? That is, do flocks obey the Mermin-Wagner theorem?

To answer this, we need to calculate the real-space, real-time fluctuations $\left\langle|\vec{v}(\vec{r}, t)|^{2}\right\rangle$.

To have true long-ranged orientational order, which is necessary to have an ordered, coherently moving flock, these fluctuations must remain finite as the size of the flock goes to infinity. To calculate $\left\langle|\vec{v}(\vec{r}, t)|^{2}\right\rangle$ from $C_{i j}(\vec{q}, \omega)$, we must Fourier Transform back to real space $\vec{r}$ and real time $t$ from $\vec{q}$ and $\omega$ space, respectively. Going back to real time first gives the spatially Fourier transformed equal time velocity correlation function: 


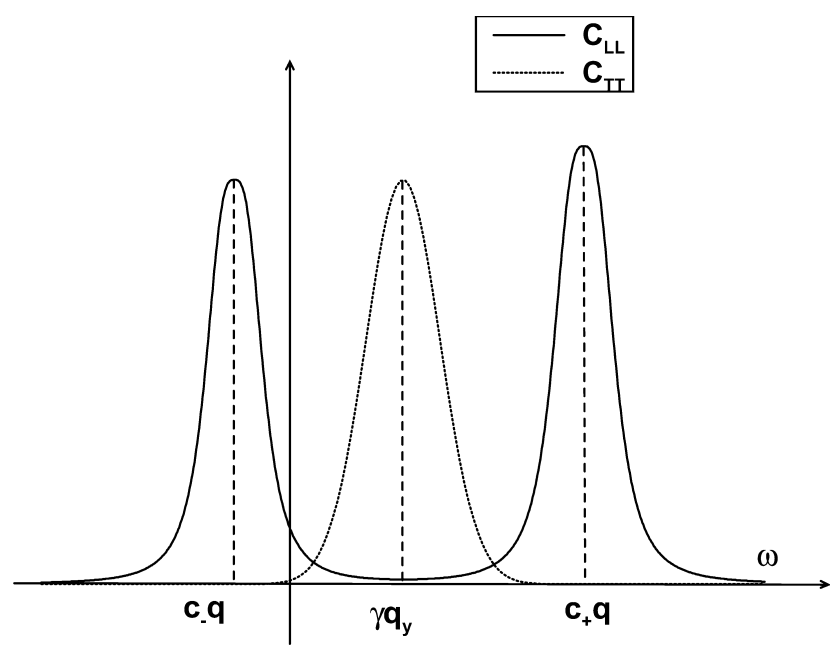

Fig. 4. Plot of $C_{\mathrm{LL}}(\vec{q}, \omega)$ and $C_{\mathrm{TT}}(\vec{q}, \omega)$ versus $\omega$ for identical fixed $\vec{q}$. Note the smallness of the overlap between the transverse and longitudinal peaks.

$$
\begin{aligned}
C_{i j}(\vec{q}) & \equiv\left\langle v_{i}(\vec{q}, t) v_{j}(-\vec{q}, t)\right\rangle \\
& =P_{i j}^{\perp}(\vec{q}) \int_{-\infty}^{\infty} \frac{\mathrm{d} \omega}{2 \pi} C_{\mathrm{TT}}(\vec{q}, \omega)+L_{i j}^{\perp}(\vec{q}) \int_{-\infty}^{\infty} \frac{\mathrm{d} \omega}{2 \pi} C_{\mathrm{LL}}(\vec{q}, \omega) \\
& =\frac{\Delta}{2}\left[\frac{P_{i j}^{\perp}(\vec{q})}{\Gamma_{\mathrm{T}}(\vec{q})}+\phi(\hat{q}) \frac{L_{i j}^{\perp}(\vec{q})}{\Gamma_{\mathrm{L}}(\vec{q})}\right] \propto \frac{1}{q^{2}},
\end{aligned}
$$

where the second integral over frequency has been evaluated in the limit of $|\vec{q}| \rightarrow 0$, so that $c\left(\theta_{\vec{q}}\right) q \gg \Gamma_{\mathrm{L}} \propto q^{2}$, and the factor $\phi(\hat{q})$ depends only on the direction $\hat{q}$ of $\vec{q}$, not its magnitude.

The quite complicated expression for $\phi(\hat{q})$ is given in [16]; for our power-counting purposes, it suffices to note that $\phi(\hat{q})$ is a smooth, analytic function of $\hat{q}$ that is $\mathrm{O}(1)$ and nonvanishing for all $\hat{q}$.

The $\frac{1}{q^{2}}$ divergence of (38) as $|\vec{q}| \rightarrow 0$ reflects the enormous long wavelength fluctuations in this system.

These fluctuations predicted by the linearized theory are strong enough to destroy long-ranged order in $d \leqslant 2$. To see this, calculate the mean squared fluctuations in $\vec{v}_{\perp}(\vec{r}, t)$ at a given point $\vec{r}$, and time $t$. This is simply the integral of the trace of Eq. (38) over all $\vec{q}$

$$
\begin{aligned}
\left\langle\left|\vec{v}_{\perp}(\vec{r}, t)\right|^{2}\right\rangle & =\int \frac{\mathrm{d}^{d} q}{(2 \pi)^{d}}\left\langle v_{i}(\vec{q}, t) v_{i}(-\vec{q},-t)\right\rangle \\
& =\frac{\Delta}{2}\left[\int \frac{\mathrm{d}^{d} q}{(2 \pi)^{d}}\left(\frac{(d-2)}{D_{\mathrm{T}} q_{\perp}^{2}+D_{\|} q_{\|}^{2}}+\frac{\phi(\hat{q})}{D_{\mathrm{L}} q_{\perp}^{2}+D_{\|} q_{\|}^{2}}\right)\right] .
\end{aligned}
$$

The last integral clearly diverges in the infrared $(|\vec{q}| \rightarrow 0)$ for $d \leqslant 2$. The divergence in the ultraviolet $(|\vec{q}| \rightarrow \infty)$ for $d \geqslant 2$ is not a concern, since we do not expect our 
theory to apply for $|\vec{q}|$ larger than the inverse of a microscopic length (such as the interaction range $\ell_{0}$ ).

The infra-red divergence in Eq. (39) for $d \leqslant 2$ cannot be dismissed so easily, since our hydrodynamic theory should get better as $|\vec{q}| \rightarrow 0$. Indeed, in the absence of nonlinear effects, this divergence is real, and signifies the destruction of long-ranged order in the linearized model by fluctuations, even for arbitrarily small noise $\Delta$, in spatial dimensions $d \leqslant 2$, and in particular in $d=2$, where the integral in Eq. (39) diverges logarithmically in the infra-red. This is so since, if $\left\langle\left|\vec{v}_{\perp}\right|^{2}\right\rangle$ is arbitrarily large even for arbitrarily small $\Delta$, our original assumption that $\vec{v}$ can be written as a mean value $\langle\vec{v}\rangle$ plus a small fluctuation $\vec{v}_{\perp}$ is clearly mistaken; indeed, the divergence of $\vec{v}_{\perp}$ suggests that the velocity can swing through all possible directions, implying that $\langle\vec{v}\rangle=0$ for $d \leqslant 2$.

In $d=2$, this result is very reminiscent of the familiar Mermin-Wagner-Hohenberg (MWH) theorem[12], which states that in equilibrium, a spontaneously broken continuous symmetry is impossible in $d=2$ spatial dimensions, precisely because of the type of logarithmic divergence of fluctuations that we have just found here. Indeed, to the linear order we ave worked here, this model looks just like an equilibrium model. All of the crucial differences between the equilibrium model and our flocking model must therefore lie in the nonlinearities. In the next section, we will show this is indeed the case: much of the scaling of correlation functions and propagators is changed from that predicted by the linearized theory in spatial dimensions $d \leqslant 4$. Most dramatically, this change in scaling makes it possible for flocks to develop long-ranged order even in $d=2$, even though equilibrium systems cannot.

\subsection{Non-linear effects and breakdown of linear hydrodynamics in the broken symmetry state}

\subsubsection{Scaling analysis}

In this section, we analyze the effect of the nonlinearities in Eqs. (12) and (13) on the long length and time behavior of the system, for spatial dimensions $d<4$. We will rescale lengths, time, and the fields $\vec{v}_{\perp}$ and $\delta \rho$ according to:

$$
\begin{aligned}
& \vec{x}_{\perp} \rightarrow b \vec{x}_{\perp}, \\
& x_{\|} \rightarrow b^{\zeta} x_{\|}, \\
& t \rightarrow b^{z} t, \\
& \vec{v}_{\perp} \rightarrow b^{\chi} \vec{v}_{\perp}, \\
& \delta \rho \rightarrow b^{\chi_{\rho}} \delta \rho .
\end{aligned}
$$

We begin by constructing the scaling which preserves the structure of the linearized theory, and then see if the nonlinearities grow or shrink under this rescaling. Accordingly, we first choose the scaling exponents to keep the diffusion constants $D_{\mathrm{B}, \mathrm{T}, \rho, \|}$, and the strength $\Delta$ of the noise fixed. The reason for choosing to keep these particular parameters fixed rather than, e.g., $\sigma_{1}$, is that these parameters completely determine the size of the equal time fluctuations in the linearized theory, as can be seen from Eq. (39). Under the rescalings (40), the diffusion constants rescale according to $D_{\mathrm{B}, \mathrm{T}} \rightarrow b^{z-2} D_{\mathrm{B}, \mathrm{T}}$ and $D_{\rho, \|} \rightarrow b^{z-2 \zeta} D_{\rho, \|}$; hence, to keep them fixed, we must choose 
$z=2$ and $\zeta=1$. The rescaling of the random force $\vec{f}$ can then be obtained from the form of the $f-f$ correlations Eq. (9) and is, for this choice of $z$ and $\zeta$

$$
\vec{f} \rightarrow b^{-1-d / 2} \vec{f} .
$$

To maintain the balance between $\vec{f}$ and the linear terms in $\vec{v}_{\perp}$ in Eq. (12), we must choose

$$
\chi=1-d / 2
$$

in Eq. (40). $\chi$ is the roughness exponent for the linearized model, i.e., we expect $\vec{v}_{\perp}$ fluctuations on length scale $L$ to scale like $L^{\chi}$. Therefore, the linearized hydrodynamic equations, neglecting the nonlinear convective terms and the nonlinearities in the pressure, imply that $\vec{v}_{\perp}$ fluctuations grow without bound (like $L^{\chi}$ ) as $L \rightarrow \infty$ for $d \leqslant 2$, where the above expression for $\chi$ becomes positive. Thus, this linearized theory predicts the loss of long range order in $d \leqslant 2$, as we saw in the last section by explicitly evaluating the real space fluctuations.

Making the rescalings as described in Eq. (40), the equation of motion (12) becomes

$$
\begin{aligned}
& \partial_{t} \vec{v}_{\perp}+b^{\gamma_{v}} \partial_{\|} \vec{v}_{\perp}+b^{\gamma_{\lambda}}\left[\lambda_{1}\left(\vec{v}_{\perp} \cdot \vec{\nabla}_{\perp}\right) \vec{v}_{\perp}+\lambda_{2}\left(\vec{\nabla}_{\perp} \cdot \vec{v}_{\perp}\right) \vec{v}_{\perp}\right] \\
& \quad=-\vec{\nabla}_{\perp}\left(\sum_{n=1}^{\infty} b^{\gamma_{n}} \sigma_{n}(\delta \rho)^{n}\right)+D_{\mathrm{B}} \vec{\nabla}_{\perp}\left(\vec{\nabla}_{\perp} \cdot \vec{v}_{\perp}\right)+D_{\mathrm{T}} \nabla_{\perp}^{2} \vec{v}_{\perp}+D_{\|} \partial_{\|}^{2} \vec{v}_{\perp}+\vec{f}_{\perp}
\end{aligned}
$$

with:

$$
\begin{aligned}
& \gamma_{\lambda}=\chi+1=2-d / 2, \\
& \gamma_{v}=z-\zeta=1
\end{aligned}
$$

and

$$
\gamma_{n}=z-\chi+n \chi-1=n+(1-n) \frac{d}{2} .
$$

The scaling exponent $\chi_{\rho}$ for $\delta \rho$ is given by $\chi_{\rho}=\chi$, since the density fluctuations $\delta \rho$ are comparable in magnitude to the $\vec{v}_{\perp}$ fluctuations. To see this, note that the eigenmode of the linearized equations of motion that involves $\delta \rho$ is a sound mode, with dispersion relation $\omega=c_{ \pm}\left(\theta_{\vec{q}}\right) q$. Inserting this into the Fourier transform of the continuity Eq. (13), we see that $\delta \rho \sim \frac{\vec{q}_{\perp} \cdot \vec{v}_{\perp}}{q_{\perp}}$. The magnitude of $\vec{q}_{\perp}$ drops out of the right hand side of this expression; hence $\delta \rho$ scales like $\left|\vec{v}_{\perp}\right|$ at long distances. Therefore, we will choose $\chi_{\rho}=\chi=1-\frac{d}{2}$.

The first two of these scaling exponents for the nonlinearities to become positive as the spatial dimension $d$ is decreased are $\gamma_{2}$ and $\gamma_{2}$, which both become positive for $d<4$, indicating that the $\lambda_{1}\left(\vec{v}_{\perp} \cdot \vec{\nabla}\right) \vec{v}_{\perp}, \lambda_{2}\left(\vec{\nabla}_{\perp} \cdot \vec{v}_{\perp}\right) \vec{v}_{\perp}$ and $\sigma_{2} \vec{\nabla}_{\perp}\left(\delta \rho^{2}\right)$ nonlinearities are all relevant perturbations for $d<4$. So, for $d<4$, the linearized hydrodynamics will break down.

A very similar breakdown of linearized hydrodynamics has long been known [28] to occur in simple equilibrium fluids for $d \leqslant 2$. Somewhat less well-known is the more dramatic, and experimentally verified, breakdown of linearized hydrodynamics that occurs in equilibrium smectic [29] and columnar [30] liquid crystals. 
What can we say about the behavior of Eqs. (12) and (13) for $d<4$, when the linearized hydrodynamics no longer holds? The answer is provided by the dynamical renormalization group, whose results we summarize in the next two sections. The first of these presents the general form of the results in arbitrary spatial dimensions $d$ with $2 \leqslant d \leqslant 4$, while the second presents results in $d=2$ exactly, in which case we can obtain exact exponents.

\subsubsection{Renormalization group analysis, $d<4$}

In this section, we summarize the results of the dynamical renormalization group analysis of the effect of the nonlinearities in the flock equations of motion. Readers interested in the details of the analysis (which are quite involved) are referred to [16].

The simplest summary of the scaling of all correlation functions and propagators is: simply use the harmonic expressions for them, except that the diffusion constants $D_{\mathrm{T}, \mathrm{B}, \rho}$ should be replaced by wavevector-dependent quantities that diverge as $\vec{q} \rightarrow 0$, according to the scaling laws

$$
D_{T, B, \rho}(\vec{q})=q_{\perp}^{z-2} f_{\mathrm{T}, \mathrm{B}, \rho}\left(\frac{\left(\frac{q_{\|}}{\Lambda}\right)}{\left(\frac{q_{\perp}}{\Lambda}\right)^{\zeta}}\right),
$$

the bare noise strength $\Delta$ should be replaced by

$$
\Delta(\vec{q})=\Delta_{*}\left(\frac{q_{\perp}}{\Lambda}\right)^{z-\zeta-2 \chi+1-d} f_{\Delta}\left(\frac{\left(\frac{q_{\|}}{\Lambda}\right)}{\left(\frac{q_{\perp}}{\Lambda}\right)^{\zeta}}\right)
$$

and the diffusion constant $D_{\|}$should be replaced by

$$
D_{\|}(\vec{q})=q_{\perp}^{z-2 \zeta} f_{\|}\left(\frac{\left(\frac{q_{\|}}{\Lambda}\right)}{\left(\frac{q_{\perp}}{\Lambda}\right)^{\zeta}}\right) .
$$

The scaling functions $f_{\Delta, \mathrm{B}, \mathrm{T}, \rho, \|}(u)$ in these expressions have the following asymptotic limits:

$$
\begin{aligned}
& f_{\mathrm{T}, \mathrm{B}, \rho}(u) \propto \begin{cases}\text { constant }, & u \rightarrow 0, \\
u^{\frac{z-2}{\zeta}}, & u \rightarrow \infty,\end{cases} \\
& f_{\Delta}(u) \propto \begin{cases}\text { constant }, & u \rightarrow 0, \\
u^{\frac{z-\zeta-2 x+1-d}{\zeta}}, & u \rightarrow \infty,\end{cases}
\end{aligned}
$$

and

$$
f_{\|}(u) \propto \begin{cases}\text { constant }, & u \rightarrow 0, \\ u^{\frac{z}{\xi}-2}, & u \rightarrow \infty .\end{cases}
$$

Here $\Lambda \sim 1 / \ell_{\mathrm{NL}}$ is an ultraviolet cutoff, with $\ell_{\mathrm{NL}}$ the length scale at which nonlinear effects become important. a one-loop RG analysis predicts: $l_{\mathrm{NL}} \sim\left(10 D_{\perp}^{5 / 4} D_{\|}^{1 / 4} /\right.$ $\left.\lambda \Delta^{1 / 2}\right)^{(2 /(4-d))} \times \mathrm{O}(1)$. Higher loop corrections may affect this result, but it presumably remains accurate to factors of $\mathrm{O}(1)$. 
The form of these scaling functions is such that the renormalized diffusion constants and the noise strength depend only on $q_{\|}$for $\frac{q_{\|}}{\Lambda} \gg\left(\frac{q_{\perp}}{\Lambda}\right)^{\zeta}$, and only on $q_{\perp}$ in the opposite limit. That is:

$$
\begin{gathered}
D_{\mathrm{T}, \mathrm{B}, \rho}(\vec{q}) \propto\left\{\begin{array}{cc}
q_{\perp}^{z-2}, & \frac{q_{\|}}{\Lambda} \ll\left(\frac{q_{\perp}}{\Lambda}\right)^{\zeta}, \\
q_{\|}^{\frac{z-2}{\zeta}}, & \frac{q_{\|}}{\Lambda} \gg\left(\frac{q_{\perp}}{\Lambda}\right)^{\zeta},
\end{array}\right. \\
\Delta(\vec{q}) \propto \begin{cases}q_{\perp}^{z-\zeta-2 \chi+1-d}, & \frac{q_{\|}}{\Lambda} \ll\left(\frac{q_{\perp}}{\Lambda}\right)^{\zeta}, \\
q_{\|}^{\frac{z-\zeta-2 \chi+1-d}{\zeta}}, & \frac{q_{\|}}{\Lambda} \gg\left(\frac{q_{\perp}}{\Lambda}\right)^{\zeta},\end{cases}
\end{gathered}
$$

and

$$
D_{\|}(\vec{q}) \propto\left\{\begin{array}{cc}
q_{\perp}^{z-2 \zeta}, & \frac{q_{\|}}{\Lambda} \ll\left(\frac{q_{\perp}}{\Lambda}\right)^{\zeta}, \\
q_{\|}^{\frac{z}{\zeta}-2}, & \frac{q_{\|}}{\Lambda} \gg\left(\frac{q_{\perp}}{\Lambda}\right)^{\zeta} .
\end{array}\right.
$$

None of the other parameters of the linearized theory is appreciably affected by the nonlinearities (beyond finite renormalizations). In particular, the sound speeds remain given by Eq. (29), with all of the parameters in that equation remaining constants as $q \rightarrow 0$.

The divergence of these diffusion "constants" and noise correlations as $q \rightarrow 0$ is the "breakdown of linearized hydrodynamics" that we argued in the last section would occur below $d=4$.

The physics of this breakdown is very simple: above $d=4$, where the breakdown does not occur, information about what is going on in one part of the flock can be transmitted to another part of the flock only by being passed sequentially through the intervening neighbors via the assumed short-ranged interactions. Below $d=4$, where the breakdown occurs, this slow, diffusive transport of information is replaced by direct, convective transport: fluctuations in the local velocity of the flock become so large, in these lower dimensions, that the motion of one part of the flock relative to another becomes the principal means of information transport, because it becomes faster than diffusion. There is a sort of "negative feedback," in that this improved transport actually suppresses the very fluctuations that give rise to it [31], leading to long-ranged order in $d=2$, as we will see in the next section.

The dynamical exponent $z$, the roughness exponent $\chi$, and the anisotropy exponent $\zeta$ completely characterize the scaling of the dynamics of flocks. Unfortunately, we have been unable to calculate them in any dimension except $d=2$. All we know is $6 / 5<z<2, \chi<\min (-1 / 5,1-d / 2)$, and $3 / 5<\zeta<1$ for $2<d<4$.

The origin of our uncertainty is the $\lambda_{2}$ term in Eq. (12). When $\lambda_{2}=0$, the structure of the theory is such that we can determine the exponents $\chi, z$, and $\zeta$ exactly. (Details of the somewhat involved argument are given in [16]; here we will simply sketch the reasoning.) This is because, when $\lambda_{2}=0$, all of the relevant pieces of the remaining vertices are total $\perp$ derivatives. It is straightforward to show that an immediate consequence of this is that $\Delta$ and $D_{\|, \rho}$ acquire no graphical renormalization when $\lambda_{2}=0$. The requirement that $\Delta, D_{\|}$, and $D_{\rho}$ flow to fixed points $\left(\frac{\mathrm{d} D_{\|, \rho}}{\mathrm{d} \ell}\right)=0=\frac{\mathrm{d} \Delta}{\mathrm{d} \ell}$ leads to two 
independent exact scaling relations between the three independent exponents $\chi, z$, and $\zeta$. Requiring $\frac{\mathrm{d} D_{\|, \rho}}{\mathrm{d} \ell}=0$ implies

$$
z=2 \zeta
$$

while requiring $\frac{\mathrm{d} \Delta}{\mathrm{d} \ell}=0$ leads to

$$
z=\zeta+2 \chi+d-1 \text {. }
$$

We emphasize that we have only shown that these relations (56) and (57) hold when $\lambda_{2}=0$.

We can obtain a third independent exact scaling relation between these three exponents, and thereby determine them exactly, when $\lambda_{2}=0$, by exploiting a "pseudo-Galilean" invariance that the equations of motion (12) and (13) have when $\lambda_{1}=\lambda_{\rho}$, which leads to a exact scaling relation, namely

$$
\chi=1-z \text {. }
$$

The three relations $\left((56)-(58)\right.$ that hold when $\lambda_{2}=0$ can trivially be solved, to find the exact scaling exponents in all $d<4$ that describe flocks with $\lambda_{2}=0$ :

$$
\begin{aligned}
& \zeta=\frac{d+1}{5}, \\
& z=\frac{2(d+1)}{5},
\end{aligned}
$$

and

$$
\chi=\frac{3-2 d}{5}
$$

Note that these match continuously, at the upper critical dimension $d=4$, onto their harmonic values $\zeta=1, z=2$, and $\chi=1-\frac{d}{2}=-1$, as they should.

But can $\lambda_{2}$ be ignored? Only if it renormalizes to zero. We have performed a dynamical RG analysis of this question, and find that $\lambda_{2}$ is unrenormalized at one loop order, leading to an apparent fixed line at that order. Since we do not know what happens to $\lambda_{2}$ at higher order, all we can say at this point is that there are three possibilities:

1. At higher order, $\lambda_{2}$ renormalizes to zero. If this is the case, then Eq. (59)-(61) hold exactly, for all flocks, for $d$ in the range $2 \leqslant d \leqslant 4$. Note that these results linearly interpolate between the equilibrium results $z=2, \zeta=1$, and $\chi=1-\frac{d}{2}$ in $d=4$, and our $2 d$ results $z=6 / 5, \zeta=3 / 5$, and $\chi=-1 / 5$ in $d=2$.

2. At higher order, $\lambda_{2}$ grows upon renormalization and reaches a nonzero fixed point value $\lambda_{2}^{*}$ at some new fixed point that differs from the $\lambda_{2}=0$ fixed point we have studied previously, at which Eqs. (59)-(61) holds. The exponents $\chi, z$, and $\zeta$ would still be universal (i.e., depend only on the dimension of space $d$ ) for all flocks in this case, but those universal values would be different from Eqs. (59)-(61).

3. $\lambda_{2}$ is unrenormalized to all orders. Should this happen, $\lambda_{2}$ would parameterize a fixed line, with continuously varying values of the exponents $z, \chi$, and $\zeta$. 
We reiterate: we do not know which of the above possibilities holds for $d>2$. However, whichever holds is universal; that is, only one of the three possibilities above applies to all flocks. We do not, however, know which one that is.

In any case, the exponents $z, \chi$, and $\zeta$ completely characterize the scaling of the dynamics and fluctuations in flocks for all dimensions $d$ in the range $2 \leqslant d \leqslant 4$. This can be seen by looking at any of the quantities we calculated in our linearized treatment in Section 2.3. For example, the full dispersion relation for the sound modes is

$$
\omega_{ \pm}=c_{ \pm}\left(\theta_{\vec{q}}\right) q-\mathrm{i} q_{\perp}^{z} f_{ \pm}\left(\frac{\frac{q_{\|}}{\Lambda}}{\left(\frac{q_{\perp}}{\Lambda}\right)^{\zeta}}\right),
$$

which follows from Eq. (28) upon replacing the diffusion constants with their wavevector-dependent values in Eqs. (47) and (49). The scaling function $f_{ \pm}$in this expression obeys

$$
f_{ \pm}(u) \propto \begin{cases}\text { constant, } & u \rightarrow 0 \\ u^{z / \zeta}, & u \rightarrow \infty .\end{cases}
$$

As a result, the attenuation of sound scales like $q_{\perp}^{z}$ for $\frac{q_{\|}}{\Lambda} \ll\left(\frac{q_{\perp}}{\Lambda}\right)^{\zeta}$, and like $q_{\|}^{z / \zeta}$ for $\frac{q_{\|}}{\Lambda} \gg\left(\frac{q_{\perp}}{\Lambda}\right)^{\zeta}$.

As discussed in Section 2.3, the dispersion relations for $\omega_{ \pm}$and $\omega_{s}$ can be directly probed by measuring the spatio-temporally Fourier transformed density-density and velocity-velocity auto-correlation functions $C_{\rho \rho}(\vec{q}, \omega)$ and $C_{i j}(\vec{q}, \omega)$.

These take exactly the same form as predicted in Section 2.3, with the replacement of the diffusion constants $D_{\rho, \mathrm{B}, \mathrm{T}}$ with the wavevector-dependent quantities given in Eqs. (47) and (49).

As a result, the two sharp peaks in $C_{\rho \rho}$ at $\omega=c_{ \pm}\left(\theta_{\vec{q}}\right) q$, now have width $\propto q_{\perp}^{z} f_{\mathrm{L}}\left(\frac{\frac{q_{\|}}{\Lambda}}{\left(\frac{q_{\perp}}{A}\right)^{\zeta}}\right)$ and height $\propto q_{\perp}^{-(2 \chi+z+3 \zeta+d-3)} g\left(\frac{\frac{q_{\|}}{\Lambda}}{\left(\frac{q_{\perp}}{A}\right)^{\zeta}}\right)$, rather than the $q^{2}$ and $q^{-4}$ scaling predicted by the linearized theory. Thus, $c_{ \pm}\left(\theta_{\vec{q}}\right)$ can be simply extracted from the position of the peaks, while the exponents $\chi, z$, and $\zeta$ can be determined by comparing their widths and heights for different $\vec{q}$ 's.

Fourier transforming $C_{i j}(\vec{q}, \omega)$ back to real time gives an equation of the same form as (38), but with $\Gamma_{\mathrm{L}, \mathrm{T}}(\vec{q})$ and $\Delta$ modified from their linearized forms by the same replacement of $D$ 's and $\Delta$ by their renormalized, wavevector-dependent values Eqs. ((47)-(49). This implies that the equal-time velocity correlation $C_{i j}(\vec{q})$ obeys the scaling laws:

$$
C_{i j}(\vec{q}) \propto \begin{cases}q_{\perp}^{z-\zeta-2 \chi+1-d}, & \frac{q_{\|}}{\Lambda} \ll\left(\frac{q_{\perp}}{\Lambda}\right)^{\zeta}, \\ \frac{z-\zeta-2 \chi+1-d}{q_{\|}^{\zeta}}, & \frac{q_{\|}}{\Lambda} \gg\left(\frac{q_{\perp}}{\Lambda}\right)^{\zeta} .\end{cases}
$$

Since $z<2$ and $\zeta<1$, these results imply that, for all $d<4$, equal-time velocity fluctuations diverge more slowly than $\frac{1}{q^{2}}$ (the latter being both the equilibrium result and that obtained by the linearized theory).

This suppression of fluctuations in Fourier space leads to fluctuations in real space that are finite, even in $d=2$, as we will show in detail in the next section. Specifically 


$$
\begin{aligned}
\left\langle\left|\vec{v}_{\perp}(\vec{r}, t)\right|^{2}\right\rangle & =\int \frac{\mathrm{d}^{d} q}{(2 \pi)^{d}}\left\langle v_{i}(\vec{q}, t) v_{i}(-\vec{q}, t)\right\rangle \\
& =\frac{1}{2} \int \frac{\mathrm{d}^{d} q}{(2 \pi)^{d}} \Delta(\vec{q})\left[\frac{(d-2)}{D_{\mathrm{T}}(\vec{q}) q_{\perp}^{2}+D_{\|}(\vec{q}) q_{\|}^{2}}+\frac{\phi(\hat{q})}{D_{\mathrm{L}}(\vec{q}) q_{\perp}^{2}+D_{\|}(\vec{q}) q_{\|}^{2}}\right]
\end{aligned}
$$

remains finite, even in $d=2$.

So far, our discussion has focussed on velocity fluctuations. The density $\rho(\vec{r}, t)$ shows huge fluctuations as well: indeed, at long wavelengths, the fluctuations of the density of birds in a flock become infinitely bigger than those in a fluid or an ideal gas. This fact is obvious to the eye in a picture of a flock. Quantitatively, Fourier transforming $C_{\rho \rho}(\vec{q}, \omega)$ back to real time yields the spatially Fourier transformed, equal time density-density correlation function $C_{\rho \rho}(\vec{q}) \equiv\left\langle|\rho(\vec{q}, t)|^{2}\right\rangle$, which obeys the scaling law:

$$
C_{\rho \rho}(\vec{q})=\frac{q_{\perp}^{3-d-\zeta-2 \chi}}{q^{2}} f_{\rho}\left(\frac{\frac{q_{\|}}{\Lambda}}{\left(\frac{q_{\perp}}{\Lambda}\right)^{\zeta}}\right) Y\left(\theta_{\vec{q}}\right) \propto \begin{cases}q_{\perp}^{1-d-\zeta-2 \chi}, & q_{\|} \ll q_{\perp}, \\ q_{\|}^{-2} q_{\perp}^{3-d-\zeta-2 \chi}, & \left(\frac{q_{\perp}}{\Lambda}\right)^{\zeta} \gg \frac{q_{\|}}{\Lambda} \gg \frac{q_{\perp}}{\Lambda}, \\ q_{\|}^{-3+\frac{1-d-2 \chi}{\zeta}} q_{\perp}^{2}, & \left(\frac{q_{\perp}}{\Lambda}\right)^{\zeta} \ll \frac{q_{\|}}{\Lambda},\end{cases}
$$

where $Y\left(\theta_{\vec{q}}\right)$ is a finite, nonvanishing, $\mathrm{O}(1)$ function of the angle $\theta_{\vec{q}}$ between the wavevector $\vec{q}$ and the direction of mean flock motion, $q_{\|}$and $\vec{q}_{\perp}$ are the wavevectors parallel and perpendicular to the broken symmetry direction, and $q_{\perp}=\left|\vec{q}_{\perp}\right|$.

The most important thing to note about $C_{\rho}(\vec{q})$ is that it diverges as $|\vec{q}| \rightarrow 0$, unlike $C_{\rho}(\vec{q})$ for, say, a simple fluid or gas, or, indeed, for any equilibrium condensed matter system, which goes to a finite constant (the compressibility) as $|\vec{q}| \rightarrow 0$.

This completes our discussion of the behavior of the full, nonlinear model in dimensions $d$ between 2 and 4. We now turn to the behavior of the model in $d=2$, where we can actually determine the exponents exactly.

\subsubsection{Ferromagnetic flock exponents in $d=2$}

In the last section, we argued that the three exponents $z, \chi$, and $\zeta$ which completely determine the scaling properties of the flock can be determined exactly if only one could show that $\lambda_{2} \rightarrow 0$ upon renormalization.

However, in $d=2$, any flock is equivalent to a flock with $\lambda_{2}=0$. This is because the $\lambda_{1}$ and $\lambda_{2}$ vertices become identical in $d=2$, where $\vec{v}_{\perp}$ has only one component, which we will take to be $x$. That is, in $d=2$ :

$$
\begin{aligned}
& \lambda_{1}\left(\vec{v}_{\perp} \cdot \vec{\nabla}_{\perp}\right) \vec{v}_{\perp}=\lambda_{1} \hat{x} v_{x} \partial_{x} v_{x}=\frac{1}{2} \lambda_{1} \partial_{x}\left(v_{x}^{2}\right) \hat{x}, \\
& \lambda_{2}\left(\vec{\nabla}_{\perp} \cdot \vec{v}_{\perp}\right) \vec{v}_{\perp}=\lambda_{2} \hat{x}\left(\partial_{x} v_{x}\right) v_{x}=\frac{1}{2} \lambda_{2} \partial_{x}\left(v_{x}^{2}\right) \hat{x},
\end{aligned}
$$

so that the full $\vec{v}_{\perp}$ nonlinearity becomes $\frac{1}{2}\left(\lambda_{1}+\lambda_{2}\right) \partial_{x}\left(v_{x}^{2}\right) \hat{x}$, which is just what we would get if we started with a (primed) model with $\lambda_{2}^{\prime}=0$ and $\lambda_{1}^{\prime}=\lambda_{1}+\lambda_{2}$. This 
latter model, since it has $\lambda_{2}^{\prime}=0$, must have the "canonical" exponents (59)-(61) hence, so must the $\left(\lambda_{1}, \lambda_{2}\right)$ model, which includes all possible $d=2$ models. So all models in $d=2$ must have the canonical exponents (59)-(61).

Setting $d=2$ in (59)-(61), we obtain:

$$
\begin{aligned}
& \zeta=\frac{3}{5}, \\
& z=\frac{6}{5}, \\
& \chi=-\frac{1}{5} .
\end{aligned}
$$

Note, in particular, that $\chi<0$. This implies, as discussed earlier, that the flock exhibits true long-ranged order.

Using the exponents (69)-(71) in the general scaling relations, such as (64) and (66), we obtain all of the scaling results for correlation functions in $d=2$. Note also that for this set of exponents $z-\zeta-2 \chi+1-d=0$. Hence, from Eq. (48), we see that the noise strength $\Delta$ is a constant, independent of $\vec{q}$, which makes sense since $\Delta$ is unrenormalized graphically. So, in the $d=2$ model, we can calculate all correlation functions from their harmonic expressions, except that we replace the diffusion constants $D_{\mathrm{B}, \mathrm{T}}$ with functions that diverge as $\vec{q} \rightarrow 0$ according to the scaling laws

$$
D_{\mathrm{B}, \mathrm{T}}(\vec{q})=q_{\perp}^{-4 / 5} f_{\mathrm{B}, \mathrm{T}}\left(\frac{\left(\frac{q_{\|}}{\Lambda}\right)}{\left(\frac{q_{\perp}}{\Lambda}\right)^{3 / 5}}\right),
$$

where we have used the exact $d=2$ exponents $z=6 / 5$ and $\zeta=3 / 5$ in the general scaling law (47). $D_{\|, \rho}$, on the other hand, are, like $\Delta$, constants, since $z=2 \zeta$ (see general Eq. (49)), which also makes sense since $D_{\|, \rho}$ are unrenormalized graphically. Hence, the only replacement needed to turn the harmonic results into the correct results for the full, nonlinear theory in $d=2$ is (72). Therefore, in $d=2, \zeta=3 / 5$, and $\chi=-1 / 5$

$$
C_{\rho \rho}(\vec{q})=\frac{q_{\perp}^{4 / 5}}{q^{2}} f_{\rho}\left(\frac{q_{\|}}{\Lambda\left(\frac{q_{\perp}}{\Lambda}\right)^{3 / 5}}\right) Y\left(\theta_{\vec{q}}\right) \propto \begin{cases}q_{\perp}^{-6 / 5}, & q_{\|} \ll q_{\perp}, \\ q_{\|}^{-2} q_{\perp}^{4 / 5}, & \left(\frac{q_{\perp}}{\Lambda}\right)^{3 / 5} \gg \frac{q_{\|}}{\Lambda} \gg \frac{q_{\perp}}{\Lambda}, \\ q_{\|}^{-4} q_{\perp}^{2}, & \left(\frac{q_{\perp}}{\Lambda}\right)^{3 / 5} \ll \frac{q_{\|}}{\Lambda} .\end{cases}
$$

These scaling predictions agree extremely well with numerical simulations $[15,16]$, as we will discuss in detail in the next section.

\section{Comparison with numerical simulations}

Many aspects of flocking dynamics are too complex to be tractable analytically. Numerical simulation thus becomes an important and necessary tool in exploring the rich phenomena in various flocking models. Even though the concept of simulat- 
ing collective behaviors in self propelled systems was introduced to the physics community by Vicsek et al. [5], computer models for flocks, not surprisingly, were used earlier in disciplines as diverse as ecology and computer graphics[1], albeit with different emphasis in each field. The main focus of the physicists in this field has been the (bulk) properties of the system in the limit of large system size, corresponding to what we refer to, at thermal equilibrium, as the thermodynamic limit. We shall use that term here as well, although we do not mean to suggest that the properties of that limit for equilibrium systems, such as equivalence of ensembles, hold here as well. We would like to understand the types of possible nonequilibrium steady states, i.e., phases of the system, and the nature of the changes from one type of phase to another when parameters are changed, i.e., the nature of the nonequilibrium phase transitions. In this section, we review numerical simulation studies in this area, with discussions of their connections to both the analytical results and the actual experimental observations, whenever possible.

\subsection{Possible behaviors (phases)}

The Vicsek model does not include any interaction to enforce a preferred distance between boids, which are kept together only by the periodic boundary conditions in the Vicsek's original study[5]. While the Vicsek model probably yields an adequate description of the transition from the moving to the nonmoving state in large systems, it cannot capture the behavior of finite flocks and of possible positional, as distinct from orientational order within the flock.

Following a model introduced by biologists Huth and Wissel [1] in the early 1990s for describing real bird flocks, the Vicsek model can be generalized by adding a central force between each pair of boids within a distance $R$ of each other. Since the main function of such interaction is to keep the flock together, it can be called the "cohesive interaction." At each time step, a total force vector is determined by summing the cohesive interaction, the velocity alignment interaction and a random noise [32] for each boid $i$

$$
\vec{f}_{i}=\sum_{j}\left(\alpha f_{a}\left(r_{i j}\right) \vec{v}_{j}+\beta f_{b}\left(r_{i j}\right) \hat{r}_{i j}\right)+\vec{\eta}_{i},
$$

where $j$ represents all the neighbors [33] within a radius $R$ centered around the $i$ th boid, $\vec{v}_{j}$ is the velocity of $j$, and $\hat{r}_{i j}$ is the unit vector from $i$ to $j . \alpha$ and $\beta$ are the strengths of the aligning and cohesive forces, respectively, and the distance-dependence is contained in the functions $f_{a, b}\left(r_{i j}\right)$, where $r_{i j}$ is the distance between boids $i$ and $j . \vec{\eta}_{i}$ is a noise with unit amplitude and random angle. At the next time step, the velocity of boid $i$ changes its direction to that of $\vec{f}_{i}$, and the boid $i$ moves with that velocity to its new position. After each boid's position and velocity have been updated, the process is repeated with the new position and velocity of the boids.

In two separate papers [34,35], the general qualitative behaviors of the flocking model with cohesive force were examined with specific choices of the interaction functions $f_{a, b}\left(r_{i j}\right)$. In [35], the strength of the alignment force is set to be constant $f_{a}\left(r_{i j}\right)=1$ and the form of the cohesive force was taken to be 

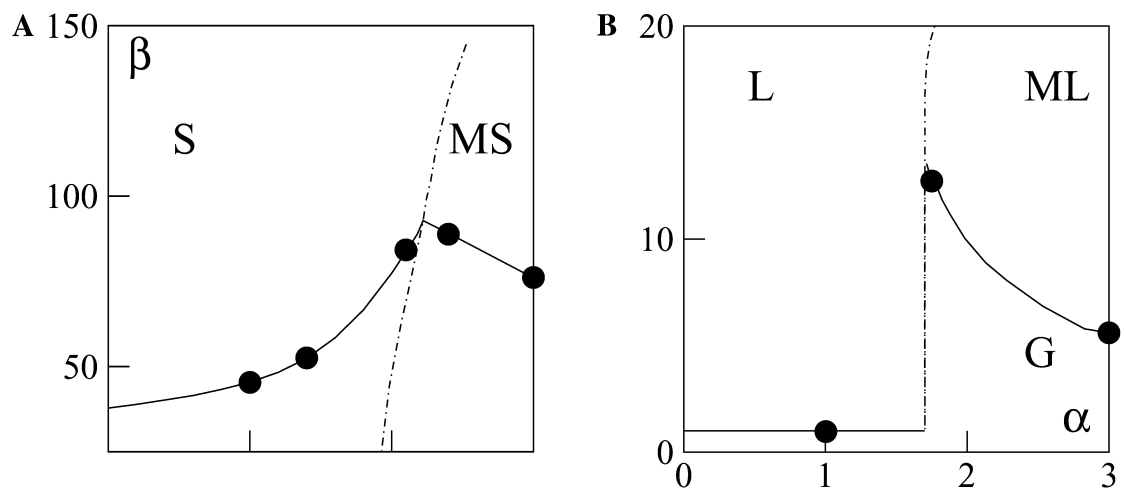

Fig. 5. Sketch of the asymptotic phase diagram in the zero-density limit. Due to the large separation of scales in $\beta$, the phase diagram is broken into two panels to show details of the transition regions. Filled circles indicate points determined numerically in [35] for a system of size $L=180, \rho=1 / 16$, see text for the parameters of the model.

$$
f_{b}\left(r_{i j}\right)= \begin{cases}-\infty: & r_{i j}<r_{c}, \\ \frac{1}{4} \frac{r_{i j}-r_{e}}{r_{a}-r_{e}}: & r_{c}<r_{i j}<r_{a}, \\ 1: & r_{a}<r_{i j}<R .\end{cases}
$$

With the parameters $r_{c}=0.2, r_{a}=0.8, r_{e}=0.5, R=1.0$, and $v_{0}=0.05$ fixed, various behaviors of the flock were then studied in the parameter space spanned by the relative strength (with respect to noise) of the cohesive $(\beta)$ and the alignment $(\alpha)$ forces in [35]. Fig. 5 summarizes the different behaviors of the system in the zero-density limit.

There are five different kinds of behaviors, which can be loosely called as "phases": moving solid (MS), stationary solid (S), moving liquid (ML), stationary liquid $(\mathrm{L})$, and the gas phase $(\mathrm{G})$. For small values of $\beta$, the boids do not stay together as a single group; instead they form smaller clusters or fly solo. We call this region the gas phase. The rest of the parameter space where the boids stay in one cluster is partitioned into four phases. In general, as $\alpha$ increases, the flock starts to move, and as $\beta$ increases, the boids seems to transform from the liquid phase, where their relative positions are constantly changing, to the solid phase, where the relative positions are fixed. In Fig. 6, the structures of these four condensed phases for a small system are shown, each with several snap shots in time, to illustrate the different flocking behaviors.

The moving and nonmoving phases can be distinguished by the average velocity of the system, as in [5]. To distinguish between the solid and the liquid phases, a relative diffusion constant $\Delta$ over some large time $T$ of initially neighboring boids was used in [35]

$$
\Delta \equiv\left\langle\frac{1}{n_{i}} \sum_{j \sim i}\left(1-\frac{r_{i j}(t)^{2}}{r_{i j}^{2}(t+T)}\right)\right\rangle_{i, t},
$$



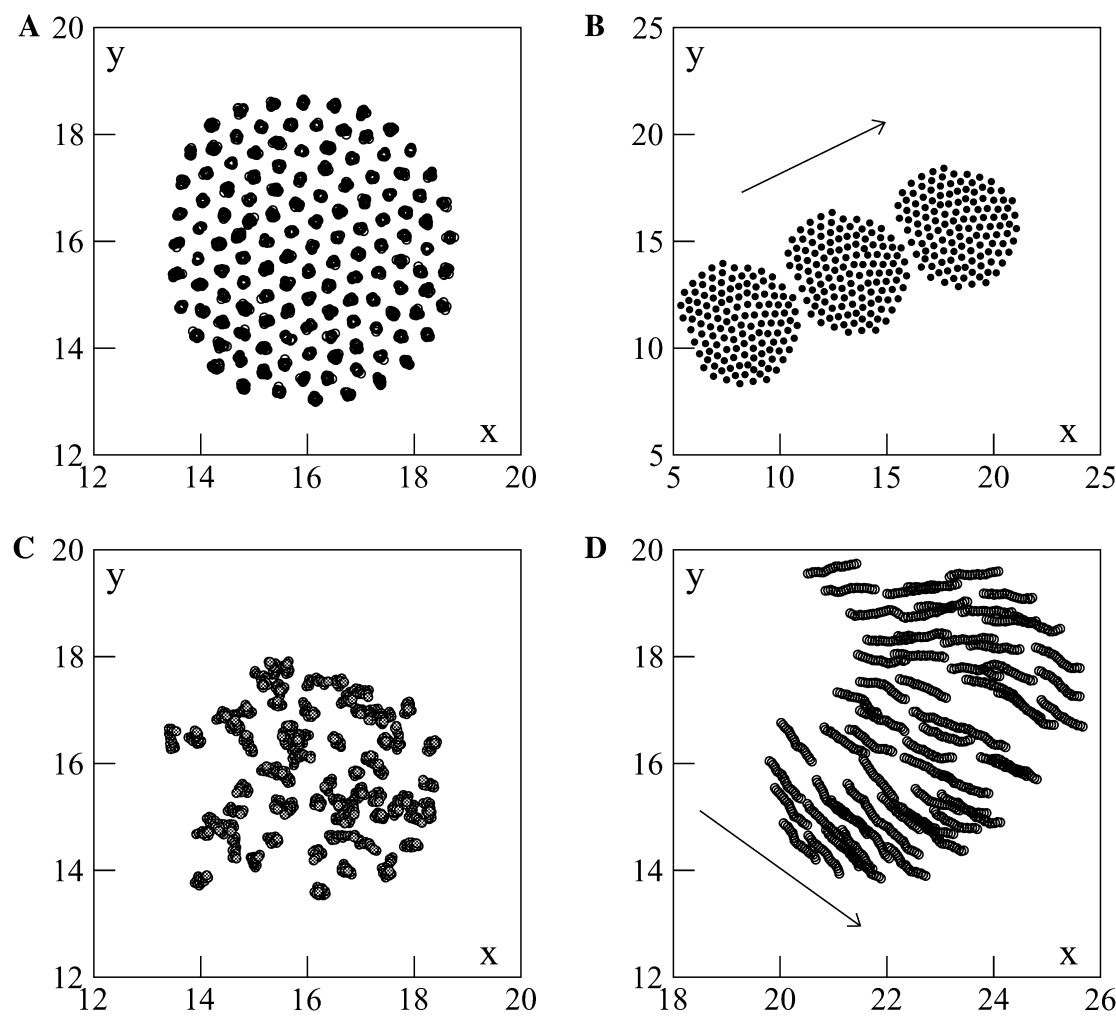

Fig. 6. Structures of condensed phases for a small system of 128 particles, and $L=32$. (A) Immobile "solid" at $\alpha=1.0$ and $\beta=100.0$ (20 timesteps superimposed). (B)"Flying crystal" at $\alpha=3.0$ and $\beta=100.0$ (three snapshots, separated by 120 timesteps). (C) Stationary fluid droplet at $\alpha=1.0$ and $\beta=2.0$ (20 consecutive timesteps). (D) Moving droplet at $\alpha=3.0$ and $\beta=3.0$ (20 consecutive timesteps). (B and D) The arrow indicates the (instantaneous) direction of motion.

where $n_{i}$ is the number of neighbors (in the Voronoi sense) for the $i$ th boid at time $t$. Time $T$ was taken to be proportional to the volume of the system, which ensures that $\Delta$ records, in the large-size limit, an asymptotic property of the system. $\Delta$ measures the inter-boid mixing inside the flock. Clearly, $\Delta \sim 1$ in the liquid phases, while $\Delta \sim 0$ for the solid phases. For finite system sizes, the transition point was chosen to be at $\Delta=\frac{1}{2}$.

It is clear that the flocking model exhibits much richer behaviors once the cohesive force is taken into account. The topology of the phase diagram is generally in agreement with our intuition and it is also evident from Fig. 5 that there are strong interactions between the two orders (positional and orientational) as reflected by the curvatures of the phase boundaries. However, to establish the existence of these phases in the thermodynamic sense requires much larger flocks and longer running time. In particular, as we see from the next section, the nature of the phase transition is very subtle, and extremely large simulation size is needed to understand it. 


\subsection{The nature of the order to disorder transition}

As described before, the global orientational order of the system is described by the average velocity of all the boids in the system. By changing the noise strength or the size of the system with constant number of boids, Vicsek et al. [5,36] found that the order parameter decreased to zero at a critical point continuously, suggesting a second order or, more generally, continuous, phase transition between a ordered and a disordered phase for the system. By doing a finite scaling analysis, a set of critical exponents were also obtained to characterize the critical point [36].

However, in a recent numerical study with much larger system size by Grégoire and Chate [37], the continuous nature of the moving to nonmoving phase transition was challenged. Grégoire and Chaté showed strong evidence for a discontinuous transition between the ordered and disordered phases of the Vicsek model. The results of their simulation are summarized in Fig. 7. From their simulation of large systems, the disordered phase can be best described as many finite clusters of boids,
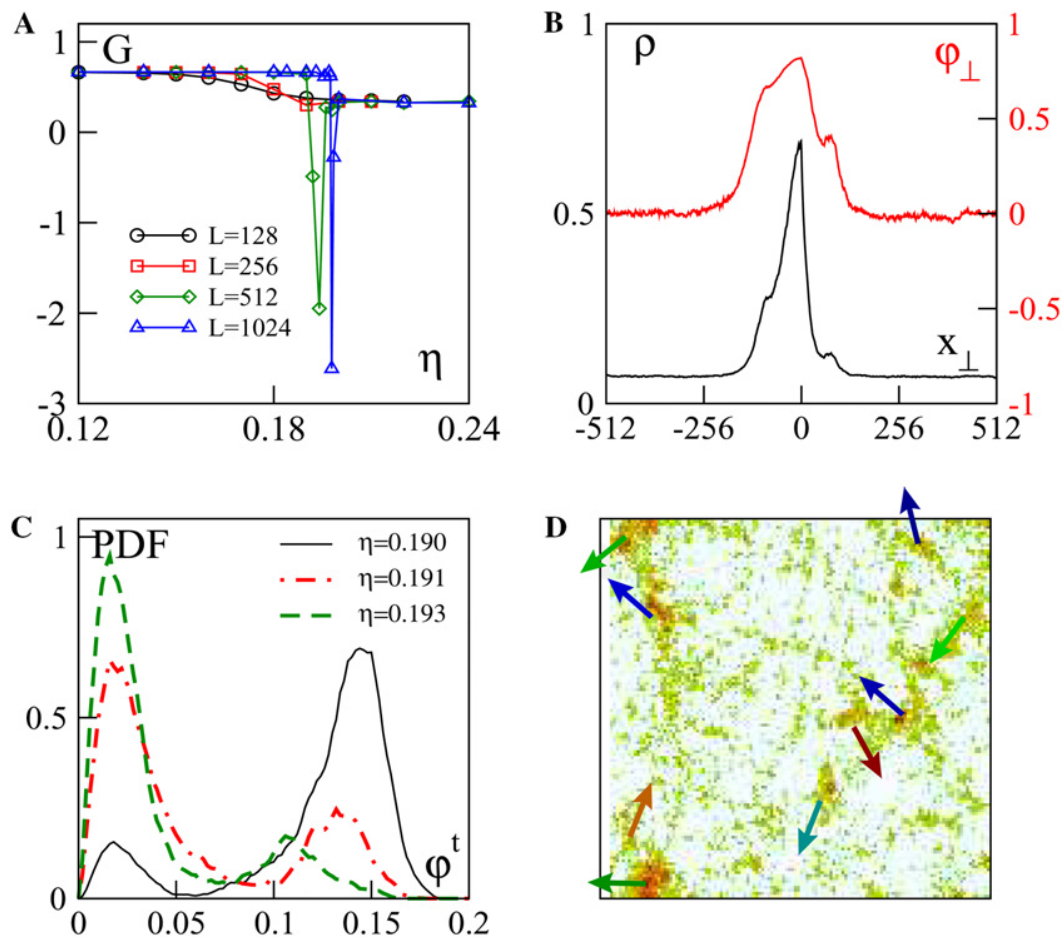

Fig. 7. Discontinuous character of the onset of collective motion in the original Vicsek model at $\rho=1 / 8$. (A) The Binder cumulant $G \equiv 1-\left\langle\phi^{4}\right\rangle / 3\left\langle\phi^{2}\right\rangle^{2}$ versus noise $\eta$ at various system sizes. (B) Density (bottom curve) and velocity (top curve) profiles along the direction of motion in the ordered phase $(L=1024$, $\eta=0.18$ ). (C) PDF of the instantaneous order parameter $\phi^{t}$ near the transition point, $t \in[\tau, 500 \tau] ; \tau \simeq 10^{5}$ is the correlation (persistent) time, $L=512$. (D) Snapshot of coarse-grained density field in disordered phase at the threshold, $\rho=2, L=256$. The arrows indicate the direction of motion of dense, ordered clusters. 
each moving in a different direction, coexisting with a dilute background of statistically stationary boids (Fig. 7D). Each finite ordered cluster evolves in time by collecting and losing boids from the background, they can also nucleate from and dissolve into the background. The ordered phase, on the other hand, consists of one or a few large clusters of boids with some intriguing internal density and velocity profile (Fig. 7B). Grégoire and Chaté argued that because these two distinctive states coexist (at least near the transition point) for finite system size, these two states occur alternately in time, leading to a seemingly continuous order parameter variation, which is probably what was observed in Vicsek's original numerical simulations. As the system size increases, the persistence time for each state becomes longer and, in the thermodynamic limit, the transition becomes discontinuous.

Perhaps the strongest evidence for a discontinuous transition is provided by the bimodal distribution of the coarse-grained local order parameter observed near the transition point, as shown in Figs. 7A and C. Similar characteristics of the order parameter were also found in other variations of the Vicsek model including ones with different forms of the noise, thus substantiating the claim of a discontinuous transition. Could the discontinuous nature of the transition be a special feature of the Vicesk model due to its lack of cohesive force? To answer this question, Grégoire and Chaté [37] also investigated the moving to nonmoving transition for flocking models with cohesive interaction in large systems. Near the critical point in the disordered phase, they found the disordered phase again consists of many small clusters connected by thin filaments of boids, each small cluster moving in different directions in much the same way as in the original Vicsek model. Grégoire and Chate therefore argued that the transition between the ordered state and this "near critical" disordered state resembled the order to disordered transition in the Vicsek model, and the onset to collective motion is always discontinuous even in the presence of cohesive force.

At the phenomenological level, it is evident that the nature of the transition is governed by the strong interaction between the positional and the orientational degrees of freedom of the system. One possibility is that the transition from the (velocity) orientationally ordered phase to the disordered phase may be caused by a finite wavelength instability for the density profile. Such instability could break the extended large cluster into many finite size ones, each of which moves in a different direction, leading to the vanishing of the global order parameter. At the theoretical level, it remains a challenge to formulate the proper interaction between density fluctuation and the directional order parameter near the ordering transition, which could then be used to understand the nature of the transition analytically.

\subsection{The properties of the ordered phase}

Away from the transition point into the ordered phase, things are a little clearer. Theoretically, the ordered phase (with uniform density) is found to be stable against fluctuations caused by the spontaneous (orientational) symmetry breaking [14-16]. However, despite its simple average behavior, the stable ordered phase has highly nontrivial properties in terms of its positional and orientational fluctuations due 
to the interaction between the sound wave and the (soft) Goldstone mode. Some of the characteristics of these fluctuations, especially those in two dimensions, can be determined from the analytical analysis of the coarse grained continuum equations. In this section, we review the work done by Tu et al. [15] where the properties of both the density and velocity fluctuations in the ordered phase were studied numerically and compared directly with the predictions from the analytical work based on the continuum equations.

The model used in [15] is similar to the modified Vicsek model given in Eq. (74). At a given time $t$, the position and the direction of the velocity for each boid are given as $\left(\vec{r}_{i}(t), \theta_{i}(t)\right)$ for $i=1,2, \ldots, N$. The magnitude of the velocity is fixed: $\left|\vec{v}_{i}\right|=v_{0}$, its direction is updated at the next time step by averaging over its neighbors' moving directions

$$
\theta_{i}(t+1)=\Theta\left(\frac{1}{M} \sum_{j=1}^{M}\left(\vec{v}_{j}(t)+\vec{g}_{i j}(t)\right)+\vec{\eta}_{i}(t)\right),
$$

where $M$ is the number of neighbors for boid $i$ within radius $R: r_{i j}=\left|\vec{r}_{i}-\vec{r}_{j}\right|<R$. The extra interaction term $\vec{g}_{i j}=g_{0}\left(\vec{r}_{i}-\vec{r}_{j}\right)\left(\left(\frac{l_{0}}{r_{i j}}\right)^{3}-\left(\frac{l_{0}}{r_{i j}}\right)^{2}\right)$ makes boids repel each other when they are closer than $l_{0}$, and attract each other otherwise, with $l_{0}$ the average distance between boids in the flock. In [15], a high boid density $\rho: \rho l_{0}^{2} \sim 1$ was chosen to prevent the formation of clusters, which render the average density profile nonuniform and complicate the analysis. The noise term $\vec{\eta}_{i}(t)=\Delta v\left(\cos \left(\pi e_{i}(t)\right)\right.$, $\left.\sin \left(\pi e_{i}(t)\right)\right)$, where $e_{i}(t)$ is a random number in the interval [-1,1]. The function $\Theta(\vec{x})$ is just the polar angle of the vector $\vec{x}$. The position update is simply: $\vec{r}_{i}(t+1)=\vec{r}_{i}(t)+v_{0}\left(\cos \left(\theta_{i}(t)\right), \sin \left(\theta_{i}(t)\right)\right)$. The parameters in this model are $R, l_{0}$, $\Delta v, v_{0}$, and $g_{0}$.

The particular form of the interactions should not affect the universal predictions of the continuum theory presented above, but rather should only change nonuniversal phenomenological parameters like $c, \lambda, D_{\|}$, etc. They also affect the length scale $l_{\mathrm{NL}}$ beyond which the asymptotic long wavelength forms of the correlation functions (32) and (37) apply. Indeed, a one-loop RG analysis predicts: $l_{\mathrm{NL}} \sim\left(10\left(D_{\perp}^{\mathrm{o}}\right)^{5 / 4}\right.$ $\left.\left(D_{\|}^{\mathrm{o}}\right)^{1 / 4} / \lambda_{\mathrm{o}} \Delta_{\mathrm{o}}^{1 / 2}\right)^{(2 /(4-d))} \times \mathrm{O}(1)$, where the sub-(or super-)script o denotes the "bare," or unrenormalized, value of the corresponding parameter. Higher loop corrections may affect this result, but it presumably remains accurate to factors of $\mathrm{O}(1)$.

For the current numerical model, the parameters for the corresponding continuum equations can be estimated on dimensional grounds: $\lambda_{\mathrm{o}} \sim 1, \Delta_{\mathrm{o}} \sim(\Delta v)^{2} \frac{R^{d}}{t_{0}}$, $D_{\|}^{\mathrm{o}} \sim D_{\perp}^{\mathrm{o}} \sim \frac{R^{2}}{t_{0}}$. Inserting these estimates, one finds $l_{\mathrm{NL}} \sim R\left(\frac{10 R}{\Delta v t_{0}}\right)^{(2 / 4-d)}$. In the simulation carried out in [15], choosing units of length and time such that $R=t_{0}=1$, and taking $\Delta v \sim \Delta v_{c} \sim 1 / 3$ in these units, for $d=2$ a lower bound for $l_{\mathrm{NL}}: l_{\mathrm{NL}}>30$ was obtained. Previous simulations [36] took $\Delta v \ll 1$, and therefore have a much larger $l_{\mathrm{NL}}$. Hence, no nontrivial nonlinear effects could be observed since their systems were much smaller than $l_{\mathrm{NL}}$.

The above analysis shows that in order to test the scaling behavior with a reasonable system size, one seeks a small $l_{\mathrm{NL}}$ by increasing $\Delta v$ and decreasing the radius of interaction $R$ as much as possible without entering the disordered phase. In the study 

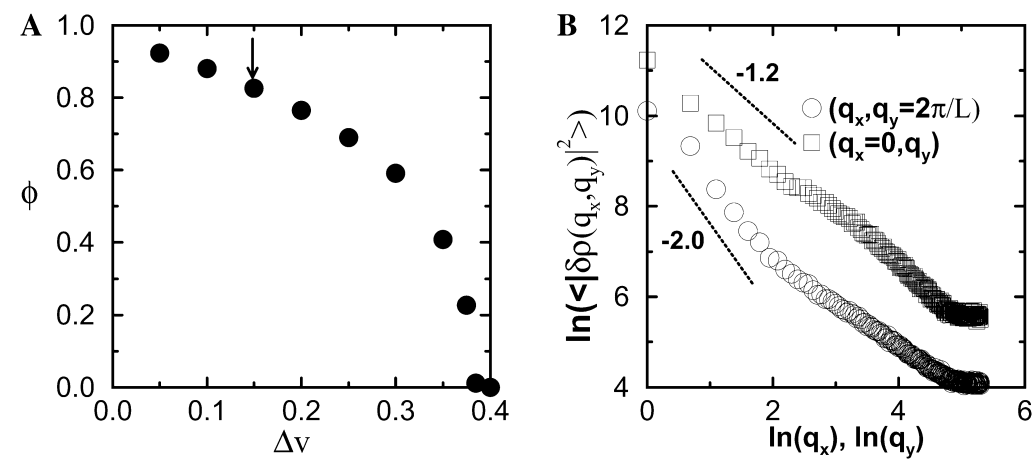

Fig. 8. (A) The order parameter $\phi$, as defined in the text, versus the noise strength $\Delta v$. The arrow shows the value of $\Delta v$ at which the fluctuations of the ordered state were calculated. (B) The scaling behavior of the equal time correlation function for the density fluctuations in the two limits can be calculated analytically (see $[14,15]$ for details) in two dimensions. The lines illustrate the predicted slopes.

by $\mathrm{Tu}$ et al. [15], the results of a simulation with system size $L \times L$ with $L=400$ and the number of boids $N=320,000$ were reported. For the parameter values $R=1$, $g_{0}=0.6, v_{0}=1.0, l_{0}=.707$ used in [15], the flock becomes disordered at $\Delta v_{c} \sim .375$ (see Fig. 8A). The order parameter $\phi$ is defined simply as the magnitude of the average velocity of the whole flock: $\phi=\frac{1}{N}\left|\sum_{i=1}^{N} \vec{v}_{i}\right|$. To stay in the ordered phase and have enough fluctuations, a large but subcritical value of noise $\Delta v=0.15$ was chosen in [15].

For any finite flock, the direction of the average velocity will slowly change, rendering difficult any comparison to the analytical results, which assume infinite system size and hence a constant direction for $\langle\vec{v}\rangle$. To make $\langle\vec{v}\rangle$ constant in its direction, periodic boundary conditions in one of the directions, say the $x$ direction, and reflecting boundary conditions in the other direction $y$ were used in [15]. When a boid $i$ with velocity $\left(v_{i}^{x}, v_{i}^{y}\right)$ collides with the "walls" at $y= \pm L / 2$, its velocity changes to $\left(v_{i}^{x},-v_{i}^{y}\right)$. The symmetry broken velocity is thus forced to lie along the $x$-direction, without changing the bulk dynamics of the system. Hereafter " $\|$ " and $x$ are used interchangeably, as are " $\perp$ " and $y$.

The equal time correlation functions can be derived analytically from the continuum model, as shown in Eq. (73), from which we see that there are three regimes of $\vec{q}$ space with different scalings of $C_{\rho \rho}(\vec{q})$ with $\vec{q}$. In Fig. $8 \mathrm{~B}$, the equal time density correlation functions are plotted in Fourier space: $C_{\rho \rho}\left(q_{\|}, q_{\perp}=2 \pi / L\right)$ versus $q_{\|}$and $C_{\rho \rho}\left(q_{\|}=0, q_{\perp}\right)$ versus $q_{\perp}$ from our simulation. The scaling behavior at long length scales can be fitted with: $C_{\rho \rho}\left(q_{\|}, q_{\perp}=2 \pi / L\right) \sim q_{\|}^{-2.05}$ and $C_{\rho \rho}\left(q_{\|}=0, q_{\perp}\right) \sim q_{\perp}^{-1.23}$. These two exponents show excellent agreement with the analytical results -2 and $-6 / 5$ predicted in Eq. (73), once one recognizes that all of the $\vec{q}$ 's in the first fit lie in the region $\left(\frac{q_{\perp}}{\Lambda}\right)^{3 / 5} \gg \frac{q_{\|}}{\Lambda} \gg \frac{q_{\perp}}{\Lambda}$, where the analytical model predicts scaling as $q_{\|}^{-2}$. As can be seen from Fig. $8 \mathrm{~B}$, the scaling region for the current simulation covers slightly less than one decade in $q_{\perp}$. It is not surprising that earlier simulations of smaller systems with less carefully chosen parameters (leading to larger $l_{\mathrm{NL}}$ ), did not observe the nontrivial scaling. 
Another interesting measurement of the simulation is the anomalous diffusion of individual boids in the direction $y$ perpendicular to the flock's moving direction. The "width" of the dispersion of an ensemble of boids: $w^{2}(t)=\left\langle\left(y_{i}(t)-y_{i}(0)\right)^{2}\right\rangle$ was measured in the simulation. The analytical behavior of the anomalous diffusion can be obtained from $w^{2}(t) \sim \int_{0}^{t} \int_{0}^{t}\left\langle v_{y}^{i}\left(t^{\prime}\right) v_{y}^{i}\left(t^{\prime \prime}\right)\right\rangle \mathrm{d} t^{\prime} \mathrm{d} t^{\prime \prime}$, where $v_{y}^{i}(t)$ is the velocity of the $i$ th boid along $y$ direction at time $t$. The velocity correlation function is given by (4):

$$
\begin{aligned}
\left\langle v_{y}^{i}(0) v_{y}^{i}(t)\right\rangle & \sim\left\langle v_{y}(\vec{x}+\phi \hat{x} t, t) v_{y}(\vec{x}, 0)\right\rangle \\
& =\int \frac{\exp \left(\mathrm{i}\left(\omega-\phi q_{\|}\right) t\right) \Delta\left(\omega-v_{s} q_{\|}\right)^{2} \mathrm{~d}^{2} q \mathrm{~d} \omega}{S(\vec{q}, \omega)} \sim t^{1-1 / \zeta}
\end{aligned}
$$

which implies: $w^{2}(t) \sim t^{3-1 / \zeta}=t^{4 / 3}$. In Fig. 9, the width squared $w^{2}(t)$ versus time $t$ from the numerical model was plotted in $\log -\log$ scale. The scaling can be fitted nicely with $w^{2}(t) \sim t^{1.3}$, which agrees well with the analytical result $t^{4 / 3}$.

Besides the scaling behavior, the analytical results (32), (37) also imply the existence of sound waves as reflected in the peaks of the correlation functions Eqs. (32) and (37). From Eq. (32), at a given value of $\vec{q}$, the correlation function has peaks at $\omega=c_{ \pm}\left(\theta_{q}\right) q$. This prediction is tested by measuring the power spectrum in the $y$ direction: $\left\langle\left|\delta \rho\left(q_{\|}=0, q_{\perp}=\frac{2 \pi}{L} n_{\perp}, \omega=\frac{2 \pi}{T} n_{\omega}\right)\right|^{2}\right\rangle(T=1024)$ with different values of $n_{\perp}(=1,2, \ldots, 20)$. Fig. 10 shows the power spectra for $n_{\perp}=5,10,20$. The spectra are symmetric around $\omega=0$ (only half of the spectrum for $\omega>0$ is shown) and the positions of the peaks $n_{\omega}^{*}$ versus $n_{y}$ are shown in the inset of Fig. 10, whose slope determines the sound velocity in the $y$-direction $c=0.62$. The power spectrum of $v_{\perp}$ in the $y$ direction was calculated also, which shows the same peaks.

Along the $x$ direction, i.e., with $q_{\|} \neq 0$ and $q_{\perp}=0$, the analytical study predicts one single peak for each correlation function. Indeed, as shown in figure Fig. 11, each power spectrum shows only one peak, and again as predicted by (32), (37), the peak for the $v_{\perp}$ power spectrum is at a different $\omega$ than the peak of the density power spec-

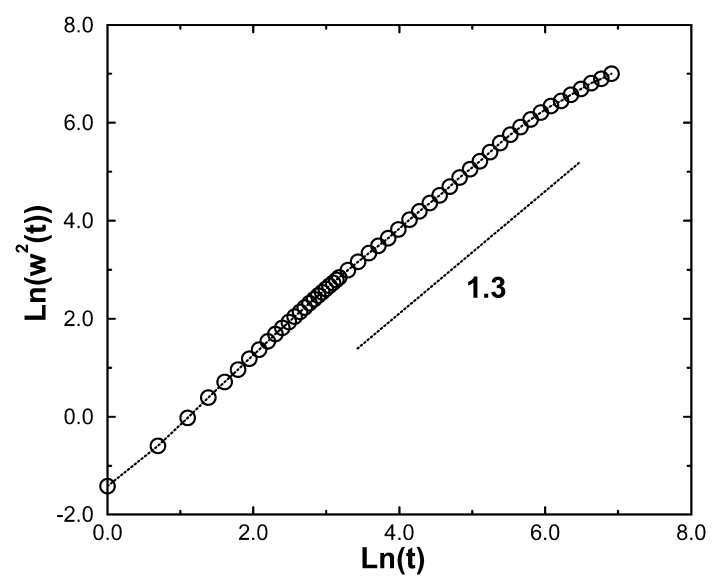

Fig. 9. The log-log plot of the anomalous transverse diffusion of an individual boid versus time. 


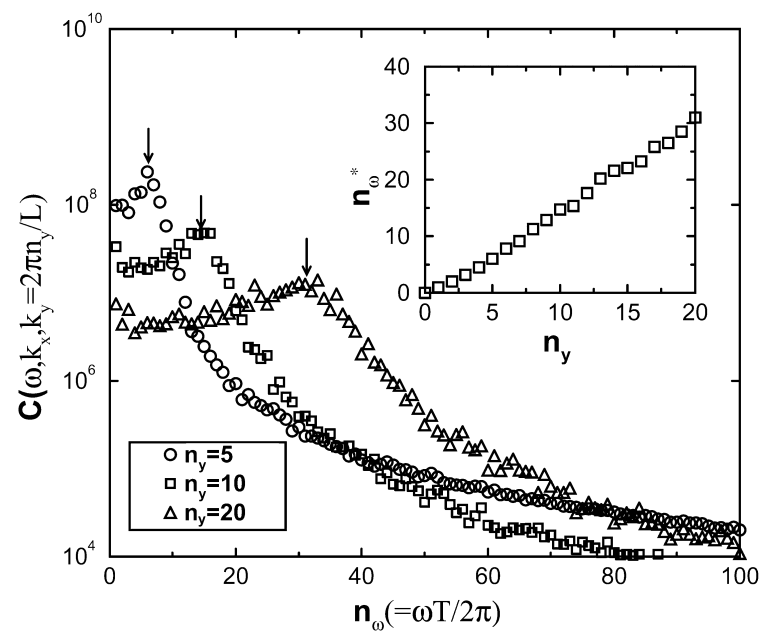

Fig. 10. The power spectrum of the density for different wave vectors. The inset shows the peak positions of the power spectrum versus wavenumber. The linear slope determines the sound velocity.

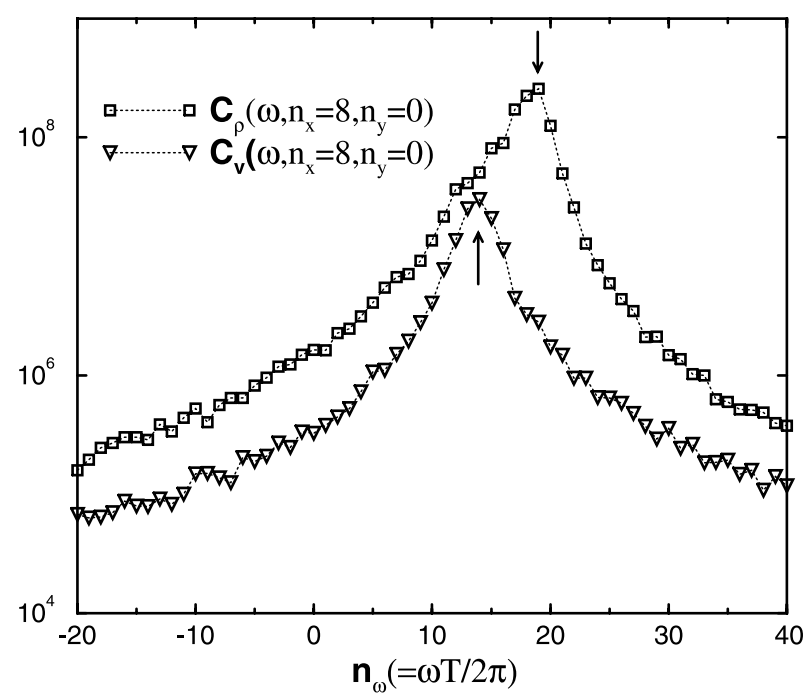

Fig. 11. Power spectra for the density and velocity fluctuations for the same wave vector along the parallel direction. The peaks of the two curves are clearly different.

trum! This means that the velocity fluctuations propagate with a different velocity than the density fluctuations in the $x$-direction!

The comparison between the numerical model and the analytical equations can be made at the quantitative level even beyond the values of exponents. From Fig. 12, the values of $v_{s}$ and $\lambda$ for the continuum model can be determined from the simula- 

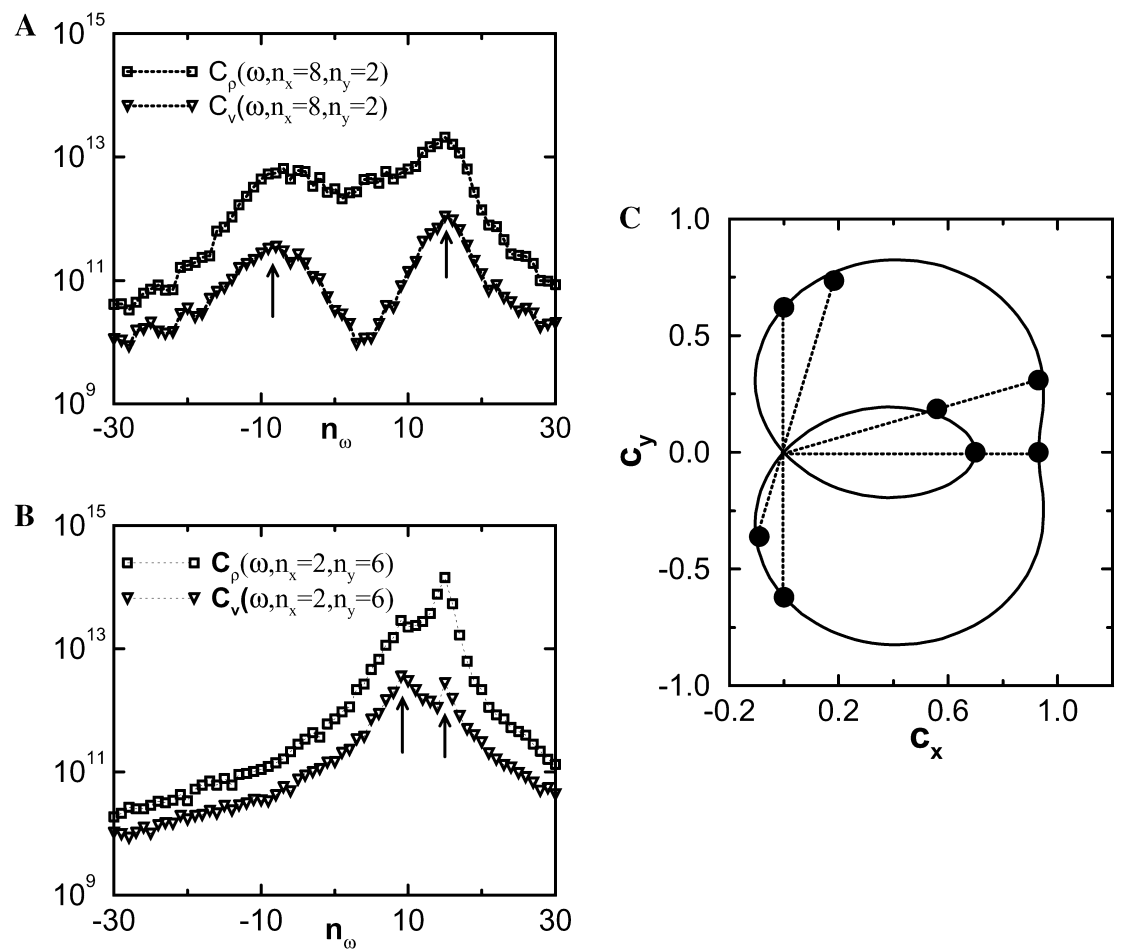

Fig. 12. The power spectra for the density and the velocity fluctuations in directions (A) $\theta_{q, 1}=\arctan (4)$ and (B) $\theta_{q, 2}=\arctan (1 / 3)$. The two peaks are clearly visible, albeit with different magnitudes. (C) The wave velocities $c_{ \pm}\left(\theta_{q}\right)$ are plotted in polar angle coordinates $\left(c_{ \pm}\left(\theta_{q}\right), \theta_{q}\right)$ for the four different directions $\theta_{q}=0, \theta_{q, 1}, \theta_{q, 2}, \pi / 2$, the two axes represent $c_{x}=c_{ \pm}\left(\theta_{q}\right) \cos \left(\theta_{q}\right)$ and $c_{y}=c_{ \pm}\left(\theta_{q}\right) \sin \left(\theta_{q}\right)$, respectively. The solid curve is the prediction from the continuum model.

tion: $v_{s}=0.93, \lambda=0.75$. (The fact that $\lambda \neq 1$ reflects the absence of Galilean invariance.) With the value of $c=0.62$ determined through Fig. 12, the sound speeds in all other directions of propagation can be predicted from Eq. (29) with no adjustable parameters. To test these predictions, the power spectra for the density and the velocity fields were calculated in the numerical model at two other angles: $\tan \left(\theta_{q}\right)=1 / 3$, 4. For the large angle $\theta_{q, 1}=\arctan (4)=76.0^{\circ}$, the data are shown in Fig. 12A. The peaks for $\rho$ and $v_{\perp}$ are at the same location, and the wave velocities are $c_{ \pm}\left(\theta_{q, 1}\right)=0.75,-0.37$. The data for $\theta_{q, 2}=\arctan (1 / 3)=18.4^{\circ}$ are shown in Fig. $12 \mathrm{~B}$. The peak at $\omega=c_{-}\left(\theta_{q, 2}\right)$ is just barely visible in the density correlation, but both peaks show very well in the velocity correlation, and the peaks for both correlation functions are at the same locations, giving the velocity $c_{ \pm}\left(\theta_{q, 2}\right)=0.97,0.59$. In Fig. $12 \mathrm{C}$, the full angle dependence of the wave velocity as predicted in Eq. (29) in polar angle coordinates $\left(c_{ \pm}\left(\theta_{q}\right), \theta_{q}\right)$ is plotted, with the values of $v_{s}, \lambda$, and $c$ determined earlier. Included in Fig. $12 \mathrm{C}$ are also the sound velocities for the two angles $\theta_{q, 1}$ and $\theta_{q, 2}$ measured in the numerical model. The agreement with the predicted velocities is excellent. 
In summary, the numerical simulations strongly support the analytical continuum theory of flocks. The observed sound speeds agree very well with predictions from the analytical model based on continuum equations of motion. In particular, the analytical model's assertion that Galilean invariance is absent is confirmed by the existence of two different nonzero sound speeds for propagation along the mean direction of flock motion. In addition, the sound attenuation shows the anomalous scaling predicted analytically.

However, all the results presented here are based on the premise that the ordered state has a homogeneous density profile, which was enforced in the numerical study in [15] by having a boid density $\rho$ compatible with the preferred inter-boid distance. If the ordered state has an inhomogeneous density profile, as reported in [37] for the Vicsek model with low boid density, the nature of the density and velocity fluctuation could be different, depending on the detailed structure of the average boid density profile.

\section{Anisotropic model}

Not all flocks, of course, are equally likely to move in any direction in the space they occupy. Flocks of birds, for instance, although they occupy a $d=3$-dimensional volume (the air), are far more likely to move horizontally than vertically. This is presumably because gravity breaks the rotational symmetry between the horizontal plane and vertical directions.

One can imagine a variety of "microscopic" rules, like the Vicsek rule described earlier, that would exhibit such anisotropy. For example, one could apply a "Vicsek" rule in three dimensions, selecting thereby a vector $\hat{n}$. Instead of moving along that vector, however, one could instead move along a vector "compressed" along some $(z)$ axis

$$
\vec{n}^{\prime}=s n_{z} \hat{z}+\vec{n}_{\perp}
$$

with $s<1$ and $\vec{n}_{\perp}=\hat{n}-n_{z} \hat{z}$. This will tend to promote motion in the $x-y$ plane at the expense of motion in the $z$-direction. Alternatively, one could project all velocities into the $x-y$ plane, apply a Vicsek rule to them (while still sampling neighbors in three dimensions), and then add to this $x y$ move a random decorrelated step in the $z$ direction [38].

In this section, we will review the work of [16] on such anisotropic models.

For technical reasons that will, we hope, become obvious, we will focus our attention on systems which, whatever their spatial dimension $d$, have an easy plane of motion; i.e., two components of velocity that are intrinsically favored over the other $d-2$. We will also assume perfect isotropy within this plane and within the $d-2$ dimensional "hard" subspace. The case of birds flying horizontally corresponds to $d=3$.

A natural extension of our fully isotropic model (EOM) to this case is

$$
\begin{aligned}
\partial_{t} \vec{v}+ & \lambda_{1}(\vec{v} \cdot \vec{\nabla}) \vec{v}+\lambda_{2}(\vec{\nabla} \cdot \vec{v}) \vec{v}+\lambda_{3} \vec{\nabla}\left(|\vec{v}|^{2}\right) \\
= & -\vec{\nabla} P(\rho)+\alpha \vec{v}-\beta|\vec{v}|^{2} \vec{v}-\delta \alpha \vec{v}_{\mathrm{H}}+D_{\mathrm{B}} \vec{\nabla}(\vec{\nabla} \cdot \vec{v})+D_{\mathrm{T}}^{e} \nabla_{\mathrm{e}}^{2} \vec{v}+D_{\mathrm{T}}^{\mathrm{H}} \nabla_{\mathrm{H}}^{2} \vec{v} \\
& +D_{2}(\vec{v} \cdot \vec{\nabla})^{2} \vec{v}+\vec{f} .
\end{aligned}
$$


Mass conservation, of course, still applies

$$
\partial_{t} \rho+\vec{\nabla} \cdot(\rho \vec{v})=0
$$

and the pressure $P(\rho)$ will still be given by the same expansion in $\delta \rho=\rho-\rho_{o}$

$$
P(\rho)=\sum_{n=1}^{\infty} \sigma_{n}(\delta \rho)^{n} .
$$

In Eq. (79), $\vec{v}_{\mathrm{H}}$ denotes the $d-2$ "hard" components of $\vec{v}$, i.e., those orthogonal to the $d=2$ easy plane. Likewise, $\nabla_{\mathrm{e}}^{2}$ and $\nabla_{\mathrm{H}}^{2}$ denote the operators $\sum_{i=1}^{2} \frac{\partial^{2}}{\partial x^{2}}$ and $\sum_{i=3}^{d} \frac{\partial^{2}}{\partial x^{2}}$, respectively, where $i=1,2$ are the "easy" Cartesian directions, and $i=3 \stackrel{\alpha}{\rightarrow} d$ the "hard" ones. The term $-\delta \alpha\left|\vec{v}_{\mathrm{H}}\right|^{2}, \delta \alpha>0$ suppresses these components relative to those in the easy plane.

Eq. (79) is not, of course, the most general anisotropic model we could write down. For instance, one could have anisotropy in the nonlinear terms: e.g, terms like $\left(\vec{v}_{\mathrm{e}} \cdot \vec{\nabla}\right) \vec{v}_{\mathrm{e}}$ could have different coefficients than $\left(\vec{v}_{\mathrm{H}} \cdot \vec{\nabla}\right) \vec{v}_{\mathrm{H}}$. However, because $\vec{v}_{\mathrm{H}}$ winds up being "massive," in the sense of decaying to zero too rapidly (i.e., nonhydrodynamically) at long wavelengths and times to nonlinearly affect the hydrodynamic (long wavelength, long time) behavior of the flock (in its low temperature phase), any additional terms in (79) distinguishing $\vec{v}_{\mathrm{H}}$ and $\vec{v}_{\mathrm{e}}$ will have no effect on the hydrodynamic behavior in the low "temperature" phase. That is, (79) already contains enough anisotropy to generate all possible relevant, symmetry allowed terms in the broken symmetry state. Hence, we will keep things simple and not generalize (79) further.

As we did for the isotropic problem, we will now break the symmetry of this model, i.e., look for solutions to the form

$$
\vec{v}(\vec{r}, t)=\langle\vec{v}\rangle+\delta \vec{v}(\vec{r}, t) \text {. }
$$

Now, however, the direction of the mean velocity $\langle\vec{v}\rangle$ (which we will chose as before, to be a static, spatially uniform solution of the noiseless $(\vec{f}=0)$ version of (79)) is not arbitrary, but must lie in the easy $(1,2)$ plane. To see this, let us, without loss of generality, write

$$
\langle\vec{v}\rangle=v_{o y} \hat{y}+v_{o z} \hat{z}
$$

with $v_{o y}$ and $v_{o z}$ constants, $\hat{y}$ in the easy plane and $\hat{z}$ one of the $d-2$ "hard" directions. To solve (79) with $\vec{f}=0$, these must obey

$$
\alpha v_{o y}-\beta\left(v_{o y}^{2}+v_{o z}^{2}\right) v_{o y}=0
$$

and

$$
(\alpha-\delta \alpha) v_{o z}-\beta\left(v_{o y}^{2}+v_{o z}^{2}\right) v_{o z}=0
$$

Subtracting $v_{o y} \times(85)$ from $v_{o z} \times$; (84) we obtain $\delta \alpha v_{o y} v_{o z}=0$, which implies that either $v_{o y}$ or $v_{o z}$ must be zero. It is straightforward to show that the former solution is unstable (with two linear eigenvalues $\alpha>0$ ) to small $\vec{v}_{\mathrm{e}}$ fluctuations, while the latter is stable (with $d-2$ linear eigenvalues $-\delta \alpha>0$ ) to $\vec{v}_{\mathrm{H}}$ fluctuations, so the solution with $\langle\vec{v}\rangle$ in the easy plane is the stable one. Furthermore, fluctuations in the "hard" 
directions are "massive," in the sense of decaying rapidly to zero even at long wavelengths, and so can be neglected in the low temperature phase (just like $v_{\|}$fluctuations in the isotropic case). Likewise, if we take

$$
\langle\vec{v}\rangle=v_{o} \hat{y}
$$

fluctuations in $\delta v_{y}=v_{y}-v_{o}$, will also be massive (with linear eigenvalue-2 - $\alpha$ ). Eliminating the massive fields $\delta v_{y}$ and $\vec{v}_{\mathrm{H}}$ in favor of the pressure, as we did for $\delta v_{\|}$in the isotropic case, gives:

$$
\begin{aligned}
& \delta v_{y}=-D_{\rho y} \partial_{y} \rho, \\
& \vec{v}_{\mathrm{H}}=-D_{\rho H} \vec{\nabla}_{\mathrm{H}} \rho,
\end{aligned}
$$

where we have defined the diffusion constants:

$$
\begin{aligned}
& D_{\rho y} \equiv \frac{\sigma_{1}}{2 \alpha}, \\
& D_{\rho H} \equiv \frac{\sigma_{1}}{\delta \alpha},
\end{aligned}
$$

and we have used the relation (81) for the pressure, and dropped all but the leading order linear terms in $\delta \rho$, since higher powers of $\delta \rho$ in Eqs. (87) and (88) prove to be irrelevant.

Using the solutions (87) and (88), and taking, for the reasons just discussed

$$
\vec{v}(\vec{r})=\left(v_{o}+\delta v_{y}(\vec{r}, t)\right) \hat{y}+v_{x}(\vec{r}, t) \hat{x}+\vec{v}_{\mathrm{H}}(\vec{r}, t)
$$

we can write a closed system of equations for $v_{x}(\vec{r}, t)$ and $\delta \rho(\vec{r}, t)$ :

$$
\begin{aligned}
& \partial_{t} \delta \rho+v_{o} \partial_{y} \delta \rho+\partial_{x}\left(\rho v_{x}\right)=\left(D_{\rho y} \partial_{y}^{2}+D_{\rho H} \nabla_{\mathrm{H}}^{2}\right) \delta \rho, \\
& \partial_{t} v_{x}+\gamma \partial_{y} v_{x}+\frac{\lambda}{2} \partial_{x}\left(v_{x}^{2}\right)=-\sigma_{1} \partial_{x}(\delta \rho)-\sigma_{2} \partial_{x}(\delta \rho)^{2}+\left(D_{\|} \partial_{y}^{2}+D_{x} \partial_{x}^{2}+D_{\mathrm{H}} \nabla_{\mathrm{H}}^{2}\right) v_{x}+f_{x},
\end{aligned}
$$

where we have defined $\lambda \equiv \lambda_{1}+\lambda_{2}$, and $\gamma=\lambda_{1} v_{0}$, and dropped irrelevant terms.

Proceeding as we did in the isotropic model, we begin by linearizing these equations, Fourier transforming them, and determining their mode structure.

The result of the first two steps is the Fourier transformed equations of motion:

$$
\begin{aligned}
& {\left[-\mathrm{i}\left(\omega-v_{o} q_{y}\right)+\Gamma_{\rho}(\vec{q})\right] \delta \rho(\vec{q}, \omega)+i q_{x} \rho_{0} v_{x}(\vec{q}, \omega)=0,} \\
& {\left[-\mathrm{i}\left(\omega-\gamma q_{y}\right)+\Gamma_{v}(\vec{q})\right] v_{x}(\vec{q}, \omega)+\mathrm{i} \sigma_{1} q_{x} \delta \rho(\vec{q}, \omega)=f_{x}(\vec{q}, \omega),}
\end{aligned}
$$

where we have defined:

$$
\begin{aligned}
& \Gamma_{\rho}(\vec{q}) \equiv D_{\rho y} q_{y}^{2}+D_{\rho H} q_{\mathrm{H}}^{2}, \\
& \Gamma_{v}(\vec{q}) \equiv D_{\|} q_{y}^{2}+D_{\mathrm{H}} q_{\mathrm{H}}^{2}+D_{x} q_{x}^{2} .
\end{aligned}
$$

Again as in the isotropic model, we first determine the eigenfrequencies $\omega(\vec{q})$ of these equations, finding 


$$
\omega_{ \pm}(\vec{q})=c_{ \pm}\left(\theta_{\vec{q}}, \phi_{\vec{q}}\right) q-\mathrm{i} \epsilon_{ \pm}(\vec{q}),
$$

where the sound speeds

$$
c_{ \pm}\left(\theta_{\vec{q}}, \phi_{\vec{q}}\right)=\frac{1}{2}\left(\gamma+v_{0}\right) \cos \theta_{\vec{q}} \pm c_{2}\left(\theta_{\vec{q}}, \phi_{\vec{q}}\right)
$$

with

$$
c_{2}\left(\theta_{\vec{q}}, \phi_{\vec{q}}\right) \equiv \sqrt{\frac{1}{4}\left(\gamma-v_{0}\right)^{2} \cos ^{2} \theta_{\vec{q}}+\sigma_{1} \rho_{0} \sin ^{2} \theta_{\vec{q}} \cos ^{2} \phi_{\vec{q}}}
$$

where $\theta_{\vec{q}}$ is the polar angle between $\vec{q}$ and the $y$-axis, and $\phi_{\vec{q}}$ is the azimuthal angle, measured relative to the $x$-axis: i.e., the angle between the projection of $\vec{q}$ orthogonal to $y$, and the $x$-axis.

A polar plot of this sound speed versus $\theta_{\vec{q}}$ for $\phi_{\vec{q}}=0$ (i.e., $\vec{q}$ in the "easy" (i.e., $x-$ $y$ ) plane) looks exactly like that for the isotropic model (Fig. 2). Indeed, any slice with fixed $\phi_{\vec{q}}$ looks qualitatively like that figure, although, as $\phi_{\vec{q}} \rightarrow \frac{\pi}{2}$ (i.e., as $\vec{q}_{\perp}$, the projection of $\vec{q}$ orthogonal to $y$, approaches orthogonality to the $x$-axis), the sound velocity profile becomes two circles with their centers on the $y$ axis and both circles passing through the origin.

The dampings $\epsilon_{ \pm}(\vec{q})$ in (98) are $0\left(q^{2}\right)$, and given by

$$
\epsilon_{ \pm}(\vec{q})= \pm \frac{c_{ \pm}\left(\theta_{\vec{q}}, \phi_{\vec{q}}\right)}{2 c_{2}\left(\theta_{\vec{q}}, \phi_{\vec{q}}\right)}\left(\Gamma_{v}(\vec{q})+\Gamma_{\rho}(\vec{q})\right) \mp \frac{v_{0} \cos \left(\theta_{\vec{q}}\right)}{2 c_{2}\left(\theta_{\vec{q}}, \phi_{\vec{q}}\right)}\left(\Gamma_{v}(\vec{q})+\frac{\gamma}{v_{0}} \Gamma_{\rho}(\vec{q})\right) .
$$

Note that, unlike the isotropic problem in $d>2$, here there are no transverse modes in any $d$ : we always have just two longitudinal Goldstone modes associated with $\delta \rho$ and $v_{x}$.

We can now again parallel our treatment of the isotropic model and calculate the correlation functions and propagators. The calculation is so similar that we will not repeat the details, but merely quote the results:

$$
\begin{aligned}
& C_{v v}(\vec{q}, \omega)=\frac{\Delta\left[\left(\omega-v_{o} q_{y}\right)^{2}+\Gamma_{\rho}^{2}(\vec{q})\right]}{|\operatorname{Den}(\vec{q}, \omega)|^{2}}, \\
& C_{\rho v}(\vec{q}, \omega)=\left\langle\delta \rho(\vec{q}, \omega) v_{x}(-\vec{q},-\omega)\right\rangle=\frac{\Delta \sigma_{1} q_{x}\left(\omega-v_{o} q_{y}-\mathrm{i} \Gamma_{\rho}(\vec{q})\right)}{|\operatorname{Den}(\vec{q}, \omega)|^{2}},
\end{aligned}
$$

and

$$
C_{\rho \rho}(\vec{q}, \omega)=\frac{\Delta \rho_{0}^{2} q_{x}^{2}}{|\operatorname{Den}(\vec{q}, \omega)|^{2}}
$$

where we have defined

$$
\begin{aligned}
\operatorname{Den}(\vec{q}, \omega)= & \left(\omega-c_{+}\left(\theta_{\vec{q}}, \phi_{\vec{q}}\right) q\right)\left(\omega-c_{-}\left(\theta_{\vec{q}}, \phi_{\vec{q}}\right) q\right)+\mathrm{i}\left[\omega\left(\Gamma_{\rho}(\vec{q})+\Gamma_{v}(\vec{q})\right)\right. \\
& \left.-q_{y}\left(v_{o} \Gamma_{v}(\vec{q})+\gamma \Gamma_{\rho}(\vec{q})\right)\right]
\end{aligned}
$$

which, of course, implies 


$$
\begin{aligned}
|\operatorname{Den}(\vec{q}, \omega)|^{2}= & \left(\omega-c_{+}\left(\theta_{\vec{q}}, \phi_{\vec{q}}\right) q\right)^{2}\left(\omega-c_{-}\left(\theta_{\vec{q}}, \phi_{\vec{q}}\right) q\right)^{2}+\left[\omega \left(\Gamma_{\rho}(\vec{q})\right.\right. \\
& \left.\left.+\Gamma_{v}(\vec{q})\right)-q_{y}\left(v_{o} \Gamma_{v}(\vec{q})+\gamma \Gamma_{\rho}(\vec{q})\right)\right]^{2} .
\end{aligned}
$$

These horrific expressions actually look quite simple when plotted as a function of $\omega$ at fixed $\vec{q}$; indeed, such a plot of $C_{v v}$ looks precisely like Fig. 3: two asymmetrical peaks, centered at $\omega=c_{ \pm}\left(\theta_{\vec{q}}, \phi_{\vec{q}}\right) q$, with widths $\epsilon_{ \pm}(\vec{q}) \propto q^{2}$.

Note that, at this linear order, everything scales as it did in the isotropic problem: peak positions $\propto q$, widths $\propto q^{2}$, and heights $\propto \frac{1}{q^{4}}$.

Continuing to blindly follow the path we trod for the isotropic problem, we can calculate the equal-time $v_{x}-v_{x}$ correlation function

$$
C_{v v}(\vec{q}) \equiv\left\langle v_{x}(\vec{q}, t) v_{x}(-\vec{q}, t)\right\rangle=\int_{-\infty}^{\infty} \frac{\mathrm{d} \omega}{2 \pi} C_{v v}(\vec{q}, \omega)=\frac{\Delta}{2} \frac{\Phi(\hat{q})}{\Gamma_{\mathrm{L}}(\vec{q})},
$$

where $\Phi(\hat{q})$ depends only on the direction $\hat{q}$ of $\vec{q}$, and is given by

$$
\begin{aligned}
\Phi(\hat{q}) \equiv & \frac{1}{c_{2}\left(\theta_{\vec{q}}, \phi_{\vec{q}}\right) q}\left[\frac{\left(c_{+}\left(\theta_{\vec{q}}, \phi_{\vec{q}}\right) q-v_{o y} q_{y}\right)^{2}}{c_{+}\left(\theta_{\vec{q}}\right) q-v_{o y} q_{y}+\left(c_{+}\left(\theta_{\vec{q}}\right) q-\lambda_{1} v_{o y} q_{y}\right) \frac{\Gamma_{\rho}}{\Gamma_{\mathrm{L}}}}\right. \\
& \left.+\frac{\left(c_{-}\left(\theta_{\vec{q}}, \phi_{\vec{q}}\right) q-v_{o y} q_{y}\right)^{2}}{c_{+}\left(\theta_{\vec{q}}, \phi_{\vec{q}}\right) q-v_{o y} q_{y}+\left(c_{-}\left(\theta_{\vec{q}}\right) q-\lambda_{1} v_{o y} q_{y}\right) \frac{\Gamma_{\rho}}{\Gamma_{\mathrm{L}}}}\right] .
\end{aligned}
$$

These fluctuations again diverge like $1 / q^{2}$ as $|\vec{q}| \rightarrow 0$, just as in the isotropic problem.

This completes our abbreviated discussion of the linearized theory of the anisotropic model. The most succinct summary of this linearized theory is that everything scales just as it did in the isotropic problem. This implies that the nonlinearities (i.e., the $\lambda$ and $\sigma_{2}$ terms in the equations of motion (92)) become relevant in and below the same upper critical dimension $d_{\mathrm{uc}}=4$ as in the isotropic problem. For $d<4$, therefore, these nonlinearities will change the long-distance behavior of the anisotropic model. We will now treat these nonlinearities using renormalization group arguments similar to those we used for the isotropic model in $d=2$. Now, however, they will work for all $d$ between 2 and 4 .

Notice that all of the nonlinearities in (92) are total $x$-derivatives, just as in the $d=2$ case for the isotropic problem. Now, however, this is true in all spatial dimensions, not just in $d=2$. (This, of course, is the reason we chose to consider precisely two "soft" components.) Thus, we will now be able to derive exact exponents in this model for all spatial dimensions. We will not go through the arguments in detail, as they are virtually identical to those in the $d=2$ case for the isotropic model, but will simply quote the conclusions:

1. There are no graphical corrections to any of the diffusion constants in (92) except $D_{x}$.

2. The stable fixed point that controls the ordered phase must have $\lambda_{\rho}^{*} \neq 0$ at least for $\lambda(0)<\lambda_{\rho}(0)$, which is a finite fraction of all flocks, and

3. $\Delta$ and $\lambda_{\rho}$ are not graphically renormalized. 
Point one suggests that, in constructing our dynamical renormalization group for (92), we should scale the $x$-direction differently from both the $y$-direction and the $d-2$ hard directions. Furthermore, since both the $y$-direction and the $d-2$ hard directions are alike in having their associated diffusion constants unrenormalized, we should scale these directions the same way. Therefore, in our renormalization group, we will rescale as follows: $x \rightarrow b x,\left(y, \vec{x}_{\mathrm{H}}\right) \rightarrow b^{\zeta}\left(y, \vec{x}_{\mathrm{H}}\right), t \rightarrow b^{z} t$. With these rescalings, the recursion relations for $D_{i}, i \neq x, \rho, \Delta$, and $\lambda_{\rho}$ become:

$$
\begin{aligned}
& \frac{\mathrm{d} D_{i}}{\mathrm{~d} l}=(z-2 \zeta) D_{i}, \quad(i \neq x), \\
& \frac{\mathrm{d} \Delta}{\mathrm{d} l}=[z-2 \chi+(1-d) \zeta-1] \Delta, \\
& \frac{\mathrm{d} \lambda_{\rho}}{\mathrm{d} l}=(\chi+z-1) \lambda_{\rho} .
\end{aligned}
$$

All three relations are exact, since none of these parameters experiences any graphical renormalization. As in the isotropic case, we want all of these parameters to flow to fixed points; this leads to three exact scaling relations between the three exponents $\chi, z$, and $\zeta$ :

$$
\begin{aligned}
& z=2 \zeta, \\
& z-2 \chi+(1-d) \zeta=1, \\
& \chi=1-z,
\end{aligned}
$$

whose solution is easily found in all $d<4$ :

$$
\begin{aligned}
& \zeta=\frac{3}{7-d}, \\
& z=\frac{6}{7-d}, \\
& \chi=\frac{1-d}{7-d} .
\end{aligned}
$$

Note that these reduce to our isotropic results in $d=2$, as they should, since the two models are identical there. They also reduce to the harmonic values $z=2, \zeta=1$, and $\chi=-1$, in $d=4$, as they should, since 4 is the upper critical dimension.

In the physically interesting case of $d=3$, we obtain:

$$
\begin{aligned}
& \zeta=\frac{3}{4}, \\
& z=\frac{3}{2}, \\
& \chi=-\frac{1}{2} .
\end{aligned}
$$


As in the isotropic case, we can use scaling arguments here to show that the effect of the nonlinearities can be fully incorporated by simply replacing $D_{x}$ everywhere it appears in the linearized expressions by the divergent, wavevector-dependent scaling form:

$$
D_{x}(\vec{q})=q_{x}^{z-2} f\left[\frac{\left(\frac{q_{y}}{\Lambda}\right)}{\left(\frac{q_{x}}{\Lambda}\right)^{\zeta}}, \frac{\left(\frac{q_{H}}{\Lambda}\right)}{\left(\frac{q_{x}}{\Lambda}\right)^{\zeta}}\right] .
$$

Doing this leads to all of the scaling laws for this anisotropic problem.

\section{Active nematics on a substrate: giant number fluctuations and anomalous long-time tails}

As we said in Section 1, uniaxial orientational order, whether in active or in passive suspensions, can be of two qualitatively different kinds, namely nematic, i.e., apolar, defined by a nonvanishing traceless symmetric tensor order parameter (with a single independent component when diagonalized, for the uniaxial case) and vector, that is, polar, defined by the nonvanishing of a vector order parameter. On symmetry grounds [39], a vector-ordered nonequilibrium steady state should have a nonzero macroscopic drift velocity $\vec{v}_{0}$, while for true nematic order $\vec{v}_{0}=0$. One can therefore take the order parameter for the vector-ordered phase to be the mean swimming velocity of the self-propelled particles themselves, as we did in Section 2 and [14-16]. A phase with nematic symmetry has no mean drift velocity and in this sense is not "self-propelled," i.e., it is not like the boids that we have discussed so far in this review or that the initial work in this field was concerned with. One might imagine that such a phase has no distinctive nonequilibrium properties, since a phase that is not drifting on average does not have an order parameter that breaks timereversal symmetry. We show [21] that this naive expectation is wrong, and that certain dynamical processes allowed by the spatial symmetry of the system but ruled out by the requirements of thermal equilibrium, introduce remarkable features in the statistical behavior of active nematics. We also suggest systems where our predictions could be tested in the laboratory.

The work summarized in this section and presented in [21] makes two striking predictions.

(i) A region of an active nematic containing on average $N$ particles, should show huge number fluctuations, with a standard deviation $\sigma_{N} \propto N^{1 / 2+1 / d}$ in $d$ space dimensions, for $N \rightarrow \infty$, in the entire active nematic phase. For a two-dimensional system, in particular, this means that $\sigma_{N} \propto N$, a standard deviation scaling as the mean. This is overwhelmingly larger than that expected for any thermal equilibrium system where, for any system with a finite compressibility, i.e., for any system away from a critical point, $\sigma_{N} \propto N^{1 / 2}$ for large $N$.

(ii) Velocity autocorrelations of a tagged particle decay anomalously slowly as functions of time $t$, as $t^{-d / 2}$ for space dimension $d \geqslant 2$. Again by contrast, in thermal equilibrium systems, such a slow long-time tail [40] occurs for tagged diffusion at 
thermal equilibrium in bulk fluids, where momentum conservation dominates, not in our systems which are confined to a substrate. The solid substrate acts as a sink for momentum, so that momentum-density fluctuations are fast, not hydrodynamic [41]. These predictions should be straightforward to test in experiments, by analyzing real-space, real-time images of nematic phases of amoeboid cells [9] or agitated layers of granular rods [22], which we discuss below.

The results summarized above show that nematic phases of rod-like powders can$n o t$ be described by an effective equilibrium statistical mechanics with a noise temperature. Active nematics are qualitatively different from their thermal equilibrium counterparts. That some monodisperse isotropic granular systems (ping-pong balls) [42] and sheared fluids [43] apparently show effective equilibrium behavior tells us that fluxes induced by distortions of broken-symmetry phases detect "nonequilibriumness" better than those in isotropic systems.

The motivation for considering active nematics comes also from recent experiments $[9,22]$ that find such phases in collections of driven particles. These two works study two very different physical systems - collections of living cells of various types [9] on a glass surface, and vertically vibrated layers of granular rods [22]. The cells involved in the experiments of [9] include melanocytes, the critters that carry human skin pigment, adipocytes (fat cells), fibroblasts (cells which form connective tissue), osteoblasts (bone cells), and granulocytes (a type of white blood cell). The first four of these, under normal circumstances, are fore-aft symmetric and thus form apolar nematic phases, while granulocytes show polar behavior, although the experimental evidence for long-ranged polar order is sparse. In [22] nematic order is discussed with polar structures forming in the nematic-isotropic interface: since the nematic and isotropic domains consist, on average, of horizontal and vertical particles, respectively, the interfacial region contains tilted particles. If these are all tilted in the same direction, as happens in the experiments of [22], there is local polar order and hence a nonzero drift velocity. Note that the cells involved in the experiments of [9] are tens to hundreds of micrometers in length, and the rods in [22] are $6 \mathrm{~mm}$ long. Thus, thermal motion plays no role in the dynamics of these particles; the noise in these systems is nonequilibrium in origin, arising from biological activity in one case and collisions and mechanical shaking in the other.

Why do we treat living cells and dead granular rods on the same footing? We remarked in Section 1 that an active particle can be thought of, mechanically, as an object endowed with an internal degree of freedom (a motor) which, when fed energy from an external source, executes some kind of cyclical motion resulting, via physical couplings, in a displacement of its center of mass. The dynamical behavior of melanocytes, for example, involves rhythmic movements of the cell body and of two long projections called dendrites which appear to be responsible for the inter-cell interaction and the level of fluctuations [9]. It is clear from this point of view why a living, shape-changing cell is an active particle, but what is the internal coordinate in the case of granular rods? A little thought will show that it is the tilt of the rod away from the horizontal. This has been analyzed in detail by numerical studies in [44]. Consider one rod in the granular layer, lofted by agitation of the solid surface on which it lies. When the rod descends, the two ends will in general not make contact 
with the surface at the same instant. As a result of static friction at the point of contact, the rod will be impelled forward (generally towards the end not yet in contact with the surface [44]). Experiments on agitated granular particles show also that [22] an agitated layer of particles with a systematic tilt in one direction behaves macroscopically like a polar-ordered phase, with a mean nonzero drift velocity, and [45] that a single, intrinsically asymmetrical granular particle moves systematically in a direction determined by its orientation. Orientationally ordered phases of particles on an air-table - a generalization of the work of [42] to the case of rod-like particles, for example-would also obey our model. Although there might be complications arising from the periodic array of air-holes, these will probably be unimportant if the array spacing is much smaller than the particle size.

Before we construct the equations of motion for the dynamics of an active apolar nematic, a few remarks are in order regarding the theoretical analysis in $[22,9]$ and in a related work [46]. Ref. [9] measures global nematic order parameters and shows striking images of classic strength $-1 / 2$ disclinations in melanocyte nematics, but attempts to fit the system into an essentially equilibrium framework which, we show below, misses some rather interesting physics. In $[22,46]$ the emphasis is entirely on the polar nature of macroscopically tilted regions. Accordingly, they account for the observations of [22] through a model closely related to that of [14-16]. In addition, none of the papers mentioned above discusses, experimentally or theoretically, the statistics of fluctuations, choosing instead to consider only average properties.

Already from the work of [14-16] discussed in the preceding sections, we know how to modify the equations of classical multicomponent spin models to describe the case where the "spin" order parameter in question is actually the local velocity of a flock, and where momentum conservation plays no important role since the motion takes place on a substrate which acts as a sink for momentum. Let us now use arguments in the same spirit to derive the most general equations of motion for the case where the order parameter is instead the traceless symmetric tensor describing apolar nematic order, retaining all relevant terms allowed by symmetry. Our aim is to discover universal experimentally measurable properties, which would distinguish an active nematic from its thermal equilibrium counterpart. Our equations and the predictions that flow from them should apply to any system of active nematogenic particles on a substrate or confined between two surfaces, including the two we have mentioned above in this section.

After that prolonged preamble, we construct our equations of motion.

Since we are working within a given phase, the nematic, and not at a continuous phase transition, the slow variables for our problem are (i) the local densities of conserved quantities and (ii) the broken-symmetry modes (which become continuous symmetry transformations in the limit of zero wavenumber). Since the solid substrate on which our active particles live is a momentum sink, the only conservation law of consequence is that for the number of particles. A description valid at sufficiently long length- and timescales [47] needs then to include only the concentration field $c(\mathbf{r}, t)$ of particles and the traceless symmetric orientational order parameter tensor $\mathbf{Q}$ with components $Q_{i j}(\mathbf{r}, t)$ at point $\mathbf{r}$ and time $t$. For uniaxial nematics, to which 
we restrict our attention here, we can write, in $d$ space dimensions, $\mathbf{Q}=[\hat{\mathbf{n}} \hat{\mathbf{n}}-(1 / d) \mathbf{I}] S$, where the unit vector $\hat{\mathbf{n}}$ is the director field specifying the local axis of orientation, I is the unit tensor, and the amplitude $S$, the conventional scalar order parameter [18], measures the degree of order. For convenience in deriving the equations of motion, we introduce a fast variable, the velocity field $\vec{v}(\mathbf{r}, t)$ of the particles, which we will later eliminate in favor of the slow variables, by going to long timescales. Number conservation states

$$
\frac{\partial c}{\partial t}=-\nabla \cdot \mathbf{j}
$$

where $\mathbf{j}=c \vec{v}$ is the number current. Newton's second law for the local momentum density $m \mathbf{j}$, where $m$ is the mass of a particle, gives

$$
m \frac{\partial \mathbf{j}}{\partial t}=-\Gamma \vec{v}-\nabla \cdot \boldsymbol{\sigma}+\mathbf{f}_{R}=-\Gamma \vec{v}-w_{\mathrm{o}} \boldsymbol{\nabla} c-w_{1} \boldsymbol{\nabla} \cdot(\mathbf{Q} c)+\mathbf{f}_{R}
$$

up to bilinear order in the fields. In (123) the $\Gamma$ term, representing friction with the substrate, is the momentum sink, and the stress tensor $\sigma$ contains the (momentumconserving) effect of interparticle interactions, the $w_{0}$ term in particular being the analogue of an osmotic pressure, $\mathbf{f}_{R}$ is a random, nonconserving, spatiotemporally white Gaussian noise, not in general of thermal origin, and the $w_{1}$ term, unique to this driven system, says that inhomogeneities in the nematic order drive mass motion.

How does this last term arise? First, in any system, by definition, inhomogeneities in $\boldsymbol{\sigma}$ produce local acceleration. The $w_{1}$ term in (123) is novel because it implies a contribution to $\sigma$ proportional to the nematic order parameter. For the present, let us accept such a term simply because symmetry allows it. In [19], as we will discuss in more detail in the next section of this review, we show that this form can be obtained by starting with a microscopic description of an active particle as a permanent force dipole. Terms of the form of the $w_{1}$ term are ruled out in thermal equilibrium nematics since the stress tensor there must be derived from functional derivatives of the free energy, which leads ultimately to a stress $\sigma$ which depends on derivatives of $\hat{\mathbf{n}}$. Such terms, although present in active nematics as well, are clearly subdominant in a gradient expansion to the $w_{1}$ term in (123) and will thus be ignored in our long-wavelength theory. Since we are considering active (i.e., nonequilibrium) nematics, we are liberated from the constraint linking $\sigma$ to functional derivatives of a rotation-invariant free-energy functional. Hence, the only constraint on allowed terms is rotation invariance of the equations of motion, which (123) respects since it is tensorial in form, both $\boldsymbol{\sigma}$ and $\mathbf{Q}$ being symmetric second rank tensors.

Let us now consider small-amplitude, long-wavelength fluctuations about the uniaxial nematic phase, aligned along the $z$ axis, denoting vector components in directions normal to $z$ by the subscript $\perp$, assuming we are deep in the nematic phase so that $S$ can be treated as constant, and considering spatial variations $c(r, t)=c_{0}+\delta c(\mathbf{r}, t)$ about the mean concentration $c_{0}$ and $\mathbf{n}(\hat{\mathbf{r}}, t)=\hat{\mathbf{z}}+\delta \mathbf{n}(\mathbf{r}, t)$ about perfect alignment. Since $\hat{\mathbf{n}}$ is a unit vector, we need consider only the transverse components $\delta n_{\perp}$. 
The equation of motion for $\hat{\mathbf{n}}$ [19], which is identical in form to that for an equilibrium nematic, reads, to linear order

$$
\partial_{t} \delta \hat{\mathbf{n}}_{\perp}=\lambda_{+} \partial_{z} \vec{v}_{\perp}+\lambda_{-} \nabla_{\perp} v_{z}+K_{1} \nabla_{\perp}\left(\nabla_{\perp} \cdot \delta \mathbf{n}_{\perp}\right)+K_{2} \nabla_{\perp}^{2} \delta \mathbf{n}_{\perp}+K_{3} \delta_{z}^{2} \delta \mathbf{n}_{\perp}+\mathbf{f}_{\perp} .
$$

Here $\lambda_{ \pm}=(\lambda \pm 1) / 2 ; \lambda$ is the "flow alignment parameter," familiar from equilibrium nematics, which affects the response of the nematic director to shear, and the noise $\mathbf{f}_{\perp}$ is delta-correlated in space and time

$$
\left\langle f_{\perp i}(\mathbf{r}, t) f_{\perp j}\left(\mathbf{r}^{\prime}, t^{\prime}\right)\right\rangle=\delta_{i j} \Delta_{n} \delta^{d}\left(\mathbf{r}-\mathbf{r}^{\prime}\right) \delta\left(t-t^{\prime}\right) .
$$

On long timescales we can neglect $m \partial_{t} \mathbf{j}$ relative to $\Gamma \vec{v}$ in (123), which allows us to treat (123) as a constitutive relation determining $\vec{v}$ in terms of the slow variables yielding, to linear order

$$
\vec{v}=-\alpha\left(\partial_{z} \delta \hat{n}_{\perp}+\left(\nabla_{\perp} \cdot \delta \hat{n}_{\perp}\right) \hat{\mathbf{z}}\right)-\gamma_{1} \nabla_{\perp} c-\gamma_{2} \partial_{z} c \hat{\mathbf{z}}+\frac{\mathbf{f}_{R}}{\Gamma},
$$

where $\alpha$ and $\gamma_{i}$ are related to $c_{o}, S$, and the coefficients in (123). Inserting (126) into (122) gives, to linear order in fluctuations, the concentration equation of motion

$$
\frac{\partial \delta c}{\partial t}=\left(D_{z} \partial_{z}^{2}+D_{\perp} \nabla_{\perp}^{2}\right) \delta c+2 c_{0} \alpha \partial_{z}\left(\nabla_{\perp} \cdot \delta \mathbf{n}_{\perp}\right)+\nabla \cdot \mathbf{f}_{c},
$$

where the noise $\mathbf{f}_{c}$ and coefficients in (127) are determined by those in (123) and (126). The linear couplings to the director in (127), a strictly nonequilibrium effect, are crucial to the analysis here, as we shall see.

The director dynamics obtained by inserting (126) into (124) is given to linear order by

$$
\frac{\partial \delta \mathbf{n}_{\perp}}{\partial t}=\left(K_{z} \partial_{z}^{2}+K_{\perp} \nabla_{\perp}^{2}+K_{L}^{\prime} \nabla_{\perp} \nabla_{\perp} \cdot\right) \delta \mathbf{n}_{\perp}+D_{c n} \partial_{z} \nabla_{\perp} \delta c+\mathbf{f}_{\perp},
$$

where, again, the phenomenological parameters and noise source $\mathbf{f}_{\perp}$ come from corresponding terms in (123)-(126).

Non-linearities such as $\boldsymbol{\nabla}_{\perp} \cdot\left(\partial_{z} \delta \mathbf{n}_{\perp} \delta c\right)$ and $\nabla_{\perp} \boldsymbol{\nabla}_{\perp}:\left(\delta \mathbf{n}_{\perp} \delta \mathbf{n}_{\perp}\right)$ are permitted in (127), and similarly $\partial_{z} \delta \mathbf{n}_{\perp}\left(\boldsymbol{\nabla}_{\perp} \cdot \delta \mathbf{n}_{\perp}\right), \delta \mathbf{n}_{\perp} \partial_{z}\left(\boldsymbol{\nabla}_{\perp} \cdot \delta \mathbf{n}_{\perp}\right)$ will in general arise in (128). Their effect remains to be sorted out in detail.

Non-linearities like the last two arise in thermal equilibrium nematics as well [48], but with prescribed relations between their coefficients because they come from differentiating anharmonic terms in the Frank free energy. Specifically in dimension $d=2$ where the director fluctuation is a single angle field $\theta$, the quadratic nonlinearities in the equation of motion of a thermal equilibrium nematic take the form $\left(K_{1}-K_{3}\right)\left[\partial_{x} \theta \partial_{z} \theta-\partial_{x} \partial_{z}(\theta)^{2}\right]$ where $K_{1}$ and $K_{3}$ are the splay and bend Frank constants. These nonlinearities contain the seeds of their own destruction: in $d=2$ they give rise to fluctuation corrections to $K_{1}$ and $K_{3}$ which initially grow logarithmically in space dimension $d=2$ as wavenumber is decreased. This growth ultimately drives the system towards a one-Frank-constant nematic, so that the strength $K_{1}-K_{3}$ of the nonlinearity is driven to zero. On asymptotically large scales, therefore, the 
Frank constants are finite and equal in thermal equilibrium nematics. The argument that two-dimensional nematics have only quasi-long-range order thus remains unaffected when the renormalization of the elastic constants is taken into account.

In active nematics these nonlinearities are not constrained to arise as the variation of a scalar functional. Preliminary results on the role of such nonlinearities in active nematics [49] suggest that they are marginally relevant in dimension $d=2$ in active nematics. Whether they result in true rather than quasi-long-range order in active nematics remains to be determined. The nonlinearities mentioned above and, more important, the concentration field itself, are absent in the treatment of Gruler et al. [9]. In what follows we stick to a strictly linearized treatment, and show how the effect of the director field on the concentration field leads to our most striking nonequilibrium effect, namely, giant number fluctuations.

We now linearize the equations of motion and look at the mode structure they imply, with an eye on possible instabilities, and then extract the statistics of small fluctuations. Let us consider modes that go as $\exp (\mathrm{iq} . \mathbf{r}-\mathrm{i} \omega t)$, and work in terms of the Fourier components $\delta c(\mathbf{q}, \omega)$ of the concentration, $f_{\mathrm{L}}(\mathbf{q}, \omega) \equiv \hat{\mathbf{q}}_{\perp} \cdot \mathbf{f}_{\perp}, \mathbf{f}_{\mathrm{T}}=\mathbf{f}_{\perp}-\hat{\mathbf{q}}_{\perp}\left(\hat{\mathbf{q}}_{\perp} \cdot \mathbf{f}_{\perp}\right)$ of the noise and $\delta \mathbf{n}_{\mathrm{L}}(\mathbf{q}, \omega) \equiv \hat{\mathbf{q}}_{\perp}\left(\hat{\mathbf{q}}_{\perp} \cdot \delta \mathbf{n}_{\perp}(\mathbf{q}, \omega)\right)$ and $\delta \mathbf{n}_{\mathrm{T}} \equiv \delta \mathbf{n}_{\perp}-\delta \mathbf{n}_{\mathrm{L}}$ of the director, the subscripts $L$ and T, respectively, denoting the components of $\mathbf{f}_{\perp}$ and $\delta \mathbf{n}_{\perp}$ along and transverse to $\hat{\mathbf{q}}_{\perp}$. We can then rewrite (127) and (128) as:

$$
\begin{aligned}
& {\left[-\mathrm{i} \omega+D_{c}(\hat{\mathbf{q}}) q^{2}\right] \delta c(\mathbf{q}, \omega)+2 c_{0} \alpha q_{z} q_{\perp} \delta n_{\mathrm{L}}(\mathbf{q}, \omega)=\mathrm{iq} \cdot \mathbf{f}_{c}(\mathbf{q}, \omega),} \\
& D_{c n} q_{z} q_{\perp} \delta c(\mathbf{q}, \omega)+\left[-\mathrm{i} \omega+K_{\mathrm{L}}(\hat{\mathbf{q}}) q^{2}\right] \delta n_{\mathrm{L}}(\mathbf{q}, \omega)=f_{\mathrm{L}}(\mathbf{q}, \omega), \\
& {\left[-\mathrm{i} \omega+K_{\mathrm{T}}(\hat{\mathbf{q}}) q^{2}\right] \delta \mathbf{n}_{\mathrm{T}}=\mathbf{f}_{\mathrm{T}},}
\end{aligned}
$$

where $\quad D_{c}(\hat{\mathbf{q}}) \equiv D_{z} \hat{q}_{z}^{2}+D_{\perp} \hat{q}_{\perp}^{2}, K_{\mathrm{L}}(\hat{\mathbf{q}}) \equiv K_{z} \hat{q}_{z}^{2}+\left(K_{\perp}+K_{\mathrm{L}}^{\prime}\right) \hat{q}_{\perp}^{2} \quad$ and $\quad K_{\mathrm{T}}(\hat{\mathbf{q}}) \equiv K_{z} \hat{q}_{z}^{2}+$ $K_{\perp} \hat{q}_{\perp}^{2}$ are direction-dependent diffusivities, whence it is straightforward to see that active nematics have $d-2$ degenerate diffusive modes (i.e., none, in two dimensions) from $\mathbf{n}_{\mathrm{T}}$, with frequencies $\omega_{\mathrm{T}}=-\mathrm{i} K_{\mathrm{T}}(\hat{\mathbf{q}}) q^{2}$ and a pair of coupled concentration-splay modes with $\omega_{ \pm}=-\mathrm{i} \Gamma_{ \pm}(\hat{\mathbf{q}}) q^{2}$, where

$$
\Gamma_{ \pm}(\hat{\mathbf{q}})=\frac{1}{2}\left[D_{c}(\hat{\mathbf{q}})+K_{\mathrm{L}}(\hat{\mathbf{q}}) \pm \sqrt{\left(D_{c}(\hat{\mathbf{q}})-K_{\mathrm{L}}(\hat{\mathbf{q}})\right)^{2}+8 c_{0} \alpha D_{c n} \hat{q}_{\perp}^{2} \hat{q}_{z}^{2}}\right] .
$$

This mode structure should be seen in contrast with that found [14-16] in Section 2 for polar ordered phases, where the interplay of the splay and concentration fields in the absence of $\hat{\mathbf{n}} \rightarrow-\hat{\mathbf{n}}$ invariance gave rise to propagating wavelike excitations with a complicated direction-dependent wavespeed. For some ranges of parameter values the eigenfrequencies we have just derived can have a positive imaginary part, implying a linear instability of the uniform nematic. We shall assume linear stability, which can be seen to be assured if the $K_{i}$ s and $D_{i}$ s are positive and $c_{0} \alpha D_{c n}$ is not too large.

With these eigenfrequencies in hand, it is straightforward to solve (129)-(131) for $\delta c, \delta n_{\mathrm{L}}$, and $\delta \mathbf{n}_{\mathrm{T}}$, autocorrelate these using the known statistical properties Eq. (125) for the random forces, and integrate over frequency to find the equal-time correlations of the fields. Unsurprisingly, we find that the variance of director fluctuations 
grows as $1 / q^{2}$ at small wavenumber $q$ (see [21] for details). Remarkably, as we have mentioned above, this leads to a similar divergence

$$
c_{0} S(\mathbf{q}) \equiv \int \frac{\mathrm{d}^{d} r}{(2 \pi)^{d}} \mathrm{e}^{-\mathrm{iq} \cdot \mathbf{r}}\langle\delta c(\mathbf{0}, t) \delta c(\mathbf{r}, t)\rangle=\frac{\left(2 c_{0} \alpha q_{\perp} q_{z}\right)^{2} \Delta_{n}}{F(\hat{\mathbf{q}}) q^{6}} \propto \frac{1}{q^{2}}
$$

in the static structure factor $S(\mathbf{q})$ for concentration fluctuations, where

$$
F(\hat{\mathbf{q}}) \equiv 2\left(D_{c}(\hat{\mathbf{q}})+K_{\mathrm{L}}(\hat{\mathbf{q}})\right)\left[D_{c}(\hat{\mathbf{q}}) K_{\mathrm{L}}(\hat{\mathbf{q}})-2 c_{0} \alpha D_{c n} \hat{q}_{\perp}^{2} \hat{q}_{z}^{2}\right]
$$

This implies colossal concentration fluctuations at small $q$, diverging as $1 / q^{2}$ in the entire nematic phase, comparable only to thermal equilibrium systems at a critical point where where the compressibility diverges as $q \rightarrow 0$, i.e., in real space, as $L^{2}$ for a system of linear dimension $L$. An additional feature in the systems of interest here is that the singularity is strongly direction-dependent, vanishing both along and perpendicular to the ordering direction, but nonvanishing for all directions in between. We have not considered what this means for response functions in the driven systems of interest; it is not completely obvious how to formulate this, since response functions require for their definition an underlying Hamiltonian, which one would need to identify for each physical realization of active nematics. Regardless of the relation between response and correlation, it is an identity that

$$
S(\mathbf{q} \rightarrow 0)=\left[\frac{\left\langle N^{2}\right\rangle-\langle N\rangle^{2}}{\langle N\rangle}\right]_{N \rightarrow \infty} .
$$

We see thus that (133) implies that the standard deviation $\sigma_{N} \propto N^{\frac{1}{2}+\frac{1}{d}}$ and in particular for $d=2$, as claimed earlier in this section, we see that $\sigma_{N} \propto N$, standard deviation proportional to the mean.

An effect as large as this should be measurable in experiments on active nematics, and would be a striking signal of their nonequilibrium nature. Two notes of caution should accompany this prediction, however. First, experimental realizations of nematics, active or otherwise, generally require quite a high particle concentration. Detecting concentration fluctuations in such dense systems could require going to very large system sizes (recall that the predictions we have made above are asymptotic results for large $N$ ). Second, our mechanism for giant fluctuations is in similar in spirit to the work of Das et al. [50] on particles sliding downhill on fluctuating surfaces with an invariance under uniform translations of the height field $h$. In active nematics, orientational fluctuations are soft and slowly relaxing because of spontaneously broken rotation-invariance; in the surface problem, the symmetry of $h \rightarrow h+$ constant means that the height field is a broken-symmetry mode. If we were to carry out a simple linearized analysis of concentration fluctuations in the problem of [50], about a state with uniform concentration and height, we would find giant concentration fluctuations with a divergent structure factor. The treatment of [50] shows that the system is actually unstable to coarsening, and the true statistical steady state at infinite time is one with a delicate kind of macroscopic phase separation. It is unclear whether that is what must ultimately happen in active nematics too. There is an important difference between the two cases: the particle velocity in the 
problem of [50] is proportional to $\nabla h$, whereas in the active nematic it is has in general a nonzero curl.

We turn next to tagged-particle motion in an active nematic. It is clear from Eq. (126) that, roughly speaking, the velocity $\dot{\mathbf{R}}$ of a tagged particle with position $\mathbf{R}(t)$ at time $t$ is a sum of contributions proportional to the values of $\nabla c$ and $\nabla \cdot \mathbf{Q}$ in its vicinity. As we have seen above, both $c$ and $\mathbf{Q}$ have diffusive dynamics and structure factors that grow as $1 / q^{2}$ at small wavenumber $q$. On long timescales their effect will thus dominate purely noise driven motions of the particle. This means the taggedparticle velocity autocorrelation has contributions schematically of the form

$$
\langle\dot{\mathbf{R}}(0) \cdot \dot{\mathbf{R}}(t)\rangle \sim\langle\phi(\mathbf{R}(0), 0) \phi(\mathbf{R}(t), t)\rangle,
$$

where $\phi$ denotes $\nabla c$ or $\boldsymbol{\nabla} \cdot \mathbf{Q}$. Using the static and dynamic correlation functions discussed above for the concentration and nematic order parameter fields, and the fact that, for a diffusing particle, $\left\langle|\mathbf{R}(t)-\mathbf{R}(0)|^{2}\right\rangle \propto t$, it is easy to see that velocity autocorrelation function (136) decays as $\int \mathrm{d}^{d} q \exp \left(-q^{2} t\right) \sim t^{-d / 2}$, as claimed earlier. Strictly speaking this analysis is internally consistent only for dimension $d>2$, since it is only then that the long-time tails can be integrated to yield a finite diffusivity. In two dimensions both tails and diffusivities will acquire multiplicative logarithmic corrections.

No experiments have yet been done to test the predictions of [21] outlined here, although several groups $[23,24]$ are currently studying mesophases in granular rods.

\section{Mode structure and instabilities of ordered active suspensions}

\subsection{General considerations and summary of results}

Thus far, we have treated our active or self-propelled particles (SPPs) as moving through a passive frictional medium. Implicitly, of course, the microscopic origin of some of the nonequilibrium terms lies in the interactions of the active particles with the medium, as discussed in [45] and in the section on active nematics on a substrate. But dynamical degrees of freedom corresponding to excitations of the medium have not made an appearance up to this point in our review. This is quite all right if one is describing migratory herds on the earth's surface, or experiments on thin layers of active particles on a solid substrate. However, it clearly leaves out a slow variable, the total momentum density of the particles plus the medium, in the case of fish or swimming bacteria. An interesting variant, which we shall not discuss here, is when the active particles are confined to the surface of a fluid medium, while their motion generates fluid flow in its bulk. In all these case, active particles speed up or slow down by exchange of momentum with the ambient fluid, leading to the well-known hydrodynamic interaction, viscous or inertial, between widely separated particles. This effect is in principle present, although perhaps weak, in birds flying through the air as well. In this section, we therefore survey such suspensions, ordered or disordered, of active particles in a fluid medium, and show how the inclusion of momentum-conserving, hydrodynamic flow into the analysis of active-particle 
systems introduces important qualitative departures from the behavior presented in the preceding sections of this review.

The problem of the individual and collective swimming of organisms has long attracted the attention of fluid-dynamicists [51-53], However, we know of no work apart from $[19,20]$ which attempts a general theory of ordered states and fluctuations therein in the context of active particles suspended in a fluid. Part of the motivation the work in $[19,20]$ came from an original experiment [54] on correlated bacterial swimming in a freely suspended fluid film, which we will discuss briefly at the end of this section, along with more recent experiments [55].

The main results of the analysis of $[19,20]$ illustrate the crucial role of the hydrodynamics of the ambient fluid medium.

(A) If the role of viscosity is ignored, as should be the case for inertia-dominated swimmers like fish, then the analysis of [19] predicts that apolar, purely nematic order in active suspensions is always destabilized at small enough wavenumber $q$, by a coupled splay of the axis of orientation and a corresponding Taylor-Couette-like circulation of the velocity field, oriented near $45^{\circ}$ to the nematic axis, with a growth rate linear in $q$. Recall, by contrast, from Section 5 and [21] that, for active nematics on a substrate, parameter ranges could always be found within which the phase was dynamically stable. Small fluctuations about a state with polar order in an active suspensions travel as propagating waves, with a most elaborate dispersion relation (see [19]), as a result of the interplay of hydrodynamic flow and the concentration with bend, twist and splay of the director field. Again, there is an important qualitative difference between these results and those obtained for polar-ordered phases of active particles on a substrate [14-16], as discussed in Section 2.3, where bend and twist displayed an overdamped rather than a propagating character.

(B) Bacterial suspensions are one natural testing ground for ideas on activeparticle systems. The appropriate dynamical regime for these ubiquitous systems is the Stokesian limit where viscous damping is dominant and inertia and accelerations are ignored. In this important limit, it was shown in [19] that a suspension in a state of uniform polar order, with nonzero mean drift velocity $\vec{v}_{0}$, is always unstable for wavevectors $\mathbf{q}$ oriented near $45^{\circ}$ to the $\vec{v}_{0}$, with a growth rate $\sim v_{0} / a$, for particles of size $a$, for $R e \ll q a \ll \phi^{1 / 2}$, where $\phi$ is the volume fraction of particles, and $R e$ is the Reynolds number evaluated at the size of a particle. The instability is "convective": it travels with a speed $\sim v_{0}$ as it grows. This result tells us that collections of coherently swimming bacteria cannot exceed a certain size $\sim a \phi^{-1 / 2}$. It is likely that this instability has been seen in [55], although the latter do not make a quantitative comparison between their observations and the predictions of [19].

(C) Isotropic suspensions of active particles are of interest for their rheology $[56,57]$, i.e., the response to imposed shear, and for the huge noise temperature they display [54]. Ref. [20] offers a framework to understand these phenomena, accounting for the large apparent temperature, predicting, as a result, hydrodynamic longtime tails with a greatly enhanced amplitude, and arguing that as the relaxation time for orientational order increases, active suspensions should display viscoelasticity of the sort seen near translational arrest in equilibrium systems. 
Lastly, number fluctuations in polar-ordered SPP suspensions are also shown to be anomalously large. The purely linearized analysis in [19] predicts precisely the same divergence as in active nematics on a substrate (Section 5 and [21]), viz. The variance $\left\langle(\delta N)^{2}\right\rangle$ in the number of particles, scaled by the mean $N$, is predicted to diverge as $N^{2 / d}$ in $d$ dimensions. This result is likely to be altered in detail once nonlinear fluctuation effects are included, as in [14-16] for polar ordered systems without fluid flow. Such an analysis has not yet been carried out for active suspensions.

\subsection{Equations of motion, modes, and instabilities}

The equations of motion from which these results follow are straightforward to construct. We begin by identifying the slow variables. The local densities of conserved quantities in an active suspension are the coarse-grained number density $c(\mathbf{r}, t)$ of active particles and the total(solute + solvent) momentum density $\mathbf{g}(\mathbf{r}, t) \equiv \rho \mathbf{u}(\mathbf{r}, t)$, where we take $\rho$, the mass density of the suspension as a wholeparticles plus fluid - to be constant since the flows in question are far slower than the speed of longitudinal sound, and $\mathbf{u}$ is the hydrodynamic velocity field. The broken symmetry variables, that is the director components $\delta \mathbf{n}_{\perp}$ perpendicular to the mean ordering direction $\hat{z}$, in uniaxial nematic or vector ordered phases have already been discussed above in Sections 2 and 5. We use the same notation as in those sections. The nematic phase is a traceless symmetric second-rank tensor $\mathbf{Q}(\mathbf{r}, t) ; Q_{i j} \propto n_{i} n_{j}-(1 / 3) \delta_{i j}$ where $\hat{\mathbf{n}}$ is the unit director field. In the ordered phase $\langle\mathbf{Q}(\mathbf{r}, t)\rangle \equiv Q_{0} \operatorname{diag}(-1,-1,2), Q_{0} \neq 0$. The presence of order in the vectorial phase is indicated by a Galilean-invariant order parameter, the nonzero average drift velocity $\langle\vec{v}(\mathbf{r}, t)\rangle=\left(0,0, v_{0}\right)$ relative to the fluid. Fluctuations about the vector ordered state are expressed in terms of the director field as $\vec{v}=\left(0,0, v_{0}\right)+v_{0} \delta \mathbf{n}_{\perp}$. When constructing the equations of motion we must keep in mind that the polar-ordered state lacks the $\hat{\mathbf{n}} \rightarrow-\hat{\mathbf{n}}$ invariance of the nematic.

We use once again the general principle that the nonequilibrium conditions oblige us to consider all terms not explicitly forbidden by symmetry or conservation, and that all invariances are to be imposed directly on the equations of motion, with no ad hoc assumptions about the existence of a free-energy functional. We impose a priori no relations amongst the phenomenological parameters other than those demanded by purely geometrical symmetries. The equations of motion follow quite simply from these considerations.

For the polar-ordered case, the director field obeys an equation which combines features of the equations for polar order on a substrate with the flow-alignment terms familiar from nematic hydrodynamics

$$
\partial_{t} \delta \mathbf{n}_{\perp}=-\lambda_{1} v_{0} \partial_{z} \delta \mathbf{n}_{\perp}-\sigma_{1} \nabla_{\perp} \delta c+\frac{1}{2}\left(\partial_{z} \mathbf{u}_{\perp}-\nabla_{\perp} u_{z}\right)+\frac{1}{2} \gamma_{2}\left(\partial_{z} \mathbf{u}_{\perp}+\nabla_{\perp} u_{z}\right)+\mathbf{D} \nabla \nabla \delta \mathbf{n}_{\perp} .
$$

In sequence, the first two terms on the right-hand side of (137), which arise in [14 16] and the discussion in Section 2, represents advection by the mean drift $v_{0}$, and 
a nonequilibrium "osmotic pressure," the third and fourth together, absent in [14 16] and Section 2, are the well-known coupling of orientation to flow $[18,58,59]$ of nematic hydrodynamics, governed by the phenomenological parameter $\gamma_{2}$, and play a central role in this section, and the last term schematically denotes director diffusion.

As in any momentum-conserving system, the momentum density $\mathbf{g}$ of particles plus fluid obeys

$$
\partial_{t} \mathbf{g}=-\nabla \cdot \boldsymbol{\sigma}
$$

where $\sigma$ is the stress tensor. The distinctive feature of active systems, as we pointed out in our discussion on active nematics on a substrate in Section 5, is a contribution to $\boldsymbol{\sigma} \propto \hat{\mathbf{n}} \hat{\mathbf{n}}-\mathbf{I} / d$ in $d$ dimensions, where $\mathbf{I}$ is the unit tensor, arising from the activity of the particles. We already argued that such a term was inevitable in driven systems simply because symmetry could not rule it out. Let us see how it arises in a more microscopic picture. Newton's third law, that the active particles and the fluid exert equal and opposite forces on each other. Thus, in the equation of motion for the total momentum density $\mathbf{g}$, the force density associated with each active particle must integrate to zero, which means that it can have no monopole moment. Averaging over the internal motions that lead to activity and self-propulsion, we see that the simplest model for, say, the $\alpha$ th active particle is a rod with axis $\hat{\mathbf{n}}_{\alpha}(t)$, at time $t$, defined by a pair of equal and opposite point forces of magnitude $f$ on its ends, directed along $\pm \hat{\mathbf{n}}_{\alpha}$ [19]. Any real active particle will of course have higher multipoles in its force distribution as well, but the monopole will be absent. In addition real active particles will not in general be uniaxial, but we restrict ourselves for simplicity here to uniaxial particles. A collection of uniaxial active particles with centers at $\mathbf{r}_{\alpha}(t)$ and ends (in general asymmetrically disposed about the center) at $\mathbf{r}_{\alpha}+a \hat{\mathbf{n}}_{\alpha}$ and $\mathbf{r}_{\alpha}-a^{\prime} \hat{\mathbf{n}}_{\alpha}$, lead to a force density (divergence of stress)

$$
\begin{aligned}
-\nabla \cdot \sigma^{(p)} & \equiv \mathbf{f}^{(p)}(\mathbf{r}, t) \\
& =f \sum_{\alpha} \hat{\mathbf{n}}_{\alpha}(t)\left[\delta\left(\mathbf{r}-\mathbf{r}_{\alpha}(t)-a \hat{\mathbf{n}}_{\alpha}(t)\right)-\delta\left(\mathbf{r}-\mathbf{r}_{\alpha}(t)+a^{\prime} \hat{\mathbf{n}}_{\alpha}(t)\right)\right] .
\end{aligned}
$$

The polar and apolar SPPs we mentioned in Section 1 correspond respectively to $a \neq a^{\prime}$ and $a=a^{\prime}$. For obvious reasons, [20] terms the former "movers" and the latter "shakers." That this force-dipole picture of active particles is physically reasonable can be seen by considering, as in [20], a simple model swimmer such as a bacterium with two flagella. This physical picture is realized as well in bundles of active filaments such as those found in the cytoskeleton [60].

Expanding the delta-functions in (139) about $\mathbf{r}_{\alpha}$ gives

$$
f_{i}^{(p)} \simeq-\frac{a+a^{\prime}}{2} f \nabla_{j} \sum_{\alpha} \hat{n}_{\alpha i} \hat{n}_{\alpha j} \delta\left(\mathbf{r}-\mathbf{r}_{\alpha}\right)+\mathrm{O}(\nabla \nabla)
$$

leading upon coarse-graining to an active contribution

$$
\sigma_{i j}^{a}(\mathbf{r}, t)=\frac{a+a^{\prime}}{2} f_{c}(\mathbf{r}, t)\left(n_{i} n_{j}-\frac{1}{3} \delta_{i j}\right)+\mathrm{O}(\nabla)
$$


to the deviatoric (traceless symmetric) stress. This justifies in detail the claim that active stresses are proportional to the local nematic order parameter. It is interesting that $a$ and $a^{\prime}$ appear only in the symmetric combination $a+a^{\prime}$ in (141); to leading order in gradients, movers and shakers have the same active stress and hence the same far-field fluid flow. A mover swims because the fluid velocity its activity induces at its own center is nonzero; the corresponding quantity is zero by symmetry for shakers. This distinction is a property of the near field fluid flow.

Using the stress (141) in the momentum Eq. (138), linearizing, Fourier-transforming in space, writing $\mathbf{g}=\rho \mathbf{u}$, and projecting transverse to the wavevector $\mathbf{q}$ to impose incompressibility $(\nabla \cdot \mathbf{u}=0)$ yields

$$
\frac{\partial \mathbf{u}_{\perp}}{\partial t}=-\mathrm{i} w_{0} q_{z}\left(\mathbf{I}-2 \frac{\mathbf{q}_{\perp} \mathbf{q}_{\perp}}{q^{2}}\right) . \delta \mathbf{n}_{\perp}-\mathrm{i} \frac{q_{z}^{2}}{q^{2}} \alpha\left(\mathbf{q}_{\perp} \delta c\right)+\boldsymbol{\eta} \nabla \nabla \mathbf{u}_{\perp},
$$

where $\boldsymbol{\eta}$ is a viscosity tensor, $\mathbf{I}$ is the unit tensor, and $\alpha \sim f a / \rho$ and $w_{0} \sim c_{0} \alpha$ are phenomenological constants proportional to the activity of the SPPs. In (142), nematic elastic torques [18], which are subdominant at small $q$, have been ignored, as have inertial and other nonlinearities. The presence of forces from concentration gradients (the pressure-like $\alpha$ term) in an incompressible system, and the acceleration proportional to the bend $q_{z} \delta \mathbf{n}_{\perp}$, are key features arising entirely because of nonequilibrium activity.

Expanding the continuity equation $\partial_{t} \delta c=-\nabla \cdot \mathbf{j}$ for the concentration for small fluctuations about the uniform ordered state, where the current $\mathbf{j}=c v_{0} \hat{\mathbf{n}}$ apart from advection by fluid flow. To leading order this leads to

$$
\left(\partial_{t}+v_{0} \partial_{z}\right) \delta c+c_{0} v_{0} \nabla_{\perp} \cdot \delta \mathbf{n}_{\perp}=0
$$

which contains advection by the mean drift $v_{0}$ and the nonequilibrium effect of local splay.

The propagating waves and instabilities mentioned at the start of this section follow from (137), (142), and (143) by considering, once again, disturbances varying as $\exp (\mathrm{iq} \cdot \mathbf{r}-\mathrm{i} \omega t)$, and assuming small wavenumber. Briefly, the coupled dynamics of bend or twist $\left(\boldsymbol{\nabla} \times \mathbf{n}_{\perp}\right)$ and vorticity along $z,\left(\boldsymbol{\nabla} \times \mathbf{u}_{\perp}\right)$, leads, from (137) and (142) to "bend-twist" waves with wavespeeds

$$
c_{\mathrm{bt}}(\theta)=\left(c_{1} \pm c_{2}\right) \cos \theta
$$

where $\theta$ is the angle between the propagation vector and the ordering direction, and $c_{1}$ and $c_{2}$ (of order the drift speed $v_{0}$ of the SPPs) are phenomenological constants. The corresponding modes in [14-16] were purely diffusive, thus highlighting the importance of fluid flow. Taking instead the divergence of (137) and (142) results in coupled equations of motion for splay $\nabla \cdot \hat{n}_{\perp}$, dilation in the $x y$ plane $\nabla \cdot \mathbf{u}_{\perp}$, and concentration fluctuations $\delta c$. The resulting wavelike eigenmodes are a rather complicated generalization of those seen in the flocking problem on a substrate [14-16], with wavespeeds determined by the solution to a cubic equation. All that really matters here is that one can show that there exists a finite range of parameter values in which the wavespeeds are real in all directions, i.e., that the system is not inevitably dynamically unstable. The speeds as functions of direction, for typical 


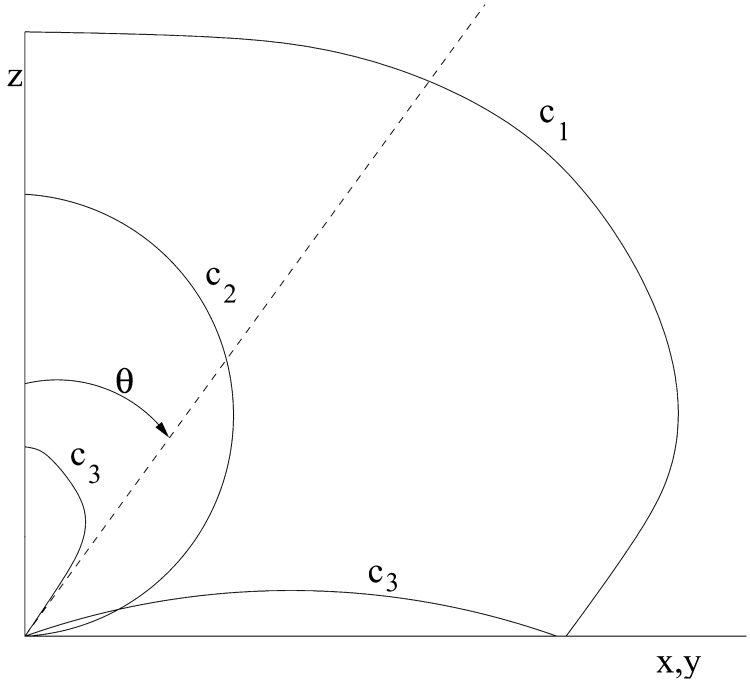

Fig. 13. Sketch of the speeds of propagating modes arising from the coupling of splay, concentration, and the $x-y$-plane dilation. The speed in a particular direction $\theta$ relative to the axis of orientation is the distance from the origin to the curve(s) in that direction.

parameter values, can be found in [19], and are plotted in Fig. 13. In all the above propagating modes, the damping, for small values of viscosity, is of order $q^{2}$.

Systems at low Reynolds number, for example bacteria, are an altogether different story. Their speeds $v_{0}$ are so low that one is in practice always in the wavenumber range where $\eta q^{2} \gg \rho v_{0} q$. We can then use the Stokesian approximation, where the velocity field $\mathbf{u}$ is determined instantaneously by a balance between viscous and stresses stresses. We can thus discard the acceleration in (142) in favor of the viscous damping term, solve for $\mathbf{u}$ in terms of concentration and director fields, and use this solution in (137) and (143). This yields effective equations of motion for the coupled dynamics of splay $\nabla \cdot \delta \mathbf{n}_{\perp}$ and $\delta c$, leading to an eigenfrequency with imaginary part

$$
\Gamma(\theta) \sim B\left(\gamma_{2} \cos 2 \theta+1\right) \cos 2 \theta
$$

with $|B| \sim f \phi / a^{2} \eta \sim v_{0} \phi / a, \phi$ being the volume fraction of active particles. Since the $\Gamma=0$ for $\theta=\pi / 4$, it must be positive (i.e., unstable) just above or just below $\theta=\pi / 4$, depending on the sign of $B$. The frequency still has a nonzero real part $\sim \pm v_{0} q \cos \theta$ : this is a convective instability, which is seen if one follows the traveling waves. Note that $\Gamma$ is independent of the magnitude of $\mathbf{q}$, as a result of the long-ranged hydrodynamic interaction in the Stokesian limit. Some algebra will show that for $q a \gtrsim \phi^{1 / 2}$, diffusivities of particles and director restabilize the mode, so the instability exists for $\mathrm{Re} \ll q a \ll \phi^{1 / 2}$. Thus, if the volume fraction is not too high, there should be a range of length scales over which vectorial order can still exist in bacterial suspensions. However, on scales of a few particle sizes, the instability should set in. It appears likely that this instability has been seen in [55], but a quantitative analysis of their experiment is awaited. 
For discussions of the instability of active apolar nematic suspensions, and for the statistics of number fluctuations, we refer the reader to [19]. Let us now move on to the rheology of active suspensions.

\subsection{Rheology of isotropic suspensions of active particles}

There have been some measurements $[56,57]$ of the dynamic elastic modulus of living cells, which suggest that active processes do make a qualitative difference to the mechanical response of matter. Although the processes taking place in a cell are complicated, from the point of view of rheology their common feature is that they involve self-generated internal forces, i.e., active stresses as discussed in the preceding section. Among the agents carrying out such processes in the cell are motor-microtubule complexes, which have successfully been modeled [61-63] using equations of motion for microtubule orientation and motor concentration related to those in [14-16] or [19]. The patterns formed in motor-microtubule extracts [61-63] suggest a strong tendency towards orientational order, so that the rheology of living cells should be describable using models such as those of the previous section on active suspensions, in the isotropic phase but nearing orientational order. We present only the simplest such approach.

Regardless of whether the particles involved are polar or apolar, the active stress, as we saw, is proportional to the local apolar order parameter. Let us assume that the proportionality constant is positive, i.e., the active stress $\boldsymbol{\sigma}^{a}=W \mathbf{Q}$, where the constant $W \sim f c_{0} a, f$ being the typical force exerted by an active particle on the fluid, $c_{0}$ the mean concentration, and $a$ the size of the active size, is primarily a single-particle property. This relation controls all the novel mechanical properties of active suspensions. We see from it why an active suspension with long-range orientational order is different from a passive system of the same symmetry. Both have $\mathbf{Q} \neq 0$, but the passive (equilibrium) phase is an equilibrium liquid despite its orientational order; Pascal's Law applies to the equilibrium nematic, so that its mean stress is strictly isotropic. An active suspension with orientational order has a nonzero mean deviatoric stress, which is a totally nonequilibrium effect. Note: an equilibrium nematogenic suspension in the isotropic phase would have a piece of the stress proportional to $\delta F / \delta \mathbf{Q}$, where $F$ is a Landau-deGennes free-energy functional. To lowest order this goes as $a \mathbf{Q}$ where the phenomenological parameter $a$ decreases as the system nears the transition to orientational order. We include this contribution in the total deviatoric stress, as well as bare viscous stresses of the form $-\eta \mathbf{A}$, where $\mathbf{A}$ is the symmetrized strain-rate tensor.

Suppose our active system shows a tendency towards orientational ordering. The relaxation time and correlation length for the nematic or polar order parameter might start to grow, but there is no reason for $W$ to vanish (or diverge) as the system approaches an ordering transition. A plausible phenomenological equation of motion for $\mathbf{Q}$ is

$$
\frac{\partial \mathbf{Q}}{\partial t}=-\frac{1}{\tau} \mathbf{Q}+D \nabla^{2} \mathbf{Q}+\lambda_{0} \mathbf{A},
$$


where $\tau$ is a relaxation time, $D$ is a diffusivity proportional, at equilibrium, to the ratio of a Frank constant to a friction constant, $\lambda_{0}$ is a flow-alignment, and $\mathbf{A}$ is the symmetrized strain-rate tensor. From the preceding discussion, we see that this leads, for sinusoidally oscillating stresses and strains $\propto \exp (-\mathrm{i} \omega t)$, to

$$
\sigma_{x y}(\omega)=-\left[\eta_{0}+\frac{(a+W) \lambda_{0}}{-\mathrm{i} \omega+\tau^{-1}}\right] A_{x y} \equiv-\frac{G^{\prime}(\omega)-\mathrm{i} G^{\prime \prime}(\omega)}{\omega} \mathrm{i} A_{x y} .
$$

Eq. (147) predicts strong viscoelasticity as $\tau$ increases. Note first that for passive (equilibrium) systems, $W=0$. Since $a \propto \tau^{-1}$ decreases as the system approaches an ordering transition, $G^{\prime}(\omega \tau \gg 1)$ decreases as $\lambda_{0} \eta_{0} / \tau$. There is not much viscoelasticity near an equilibrium isotropic-nematic transition. For active systems, as we said, $W$ is primarily a single-particle property, independent of $\tau$ and of proximity to the transition. Thus, as $\tau$ grows

$$
G^{\prime}(\omega \tau \gg 1) \simeq W
$$

independent of $\tau$ and, of course, the dynamic range over which elastic behavior is seen increases. This is classic Maxwell viscoelasticity, normally expected at equilibrium near translational not orientational ordering. The ideas on rheology proposed here have not been tested, although there has been one set of experiments [64] on optically trapped beads in a bacterial suspension in which the strength of the trapping potential was modulated. It is not obvious how to relate the theoretical developments in this section to that particular type of modulation, which is quite distinct from the microrheological measurements of, e.g. [57].

Lastly, in the isotropic phase, in the absence of imposed shear, the active stress can simply be thought of as an added noise in the momentum Eq. (138). The noise temperature corresponding to the active stress can be estimated on dimensional grounds: the variance of $\boldsymbol{\sigma}^{a}(k=0, \omega=0)$ is $\sim W^{2} \xi^{3} \tau$, with $W \sim \eta u_{0} / \xi$ for active particles moving with typical speed $u_{0}$, correlated over a scale $\xi$ and time $\tau$. We can define the effective temperature by equating this stress variance to $k_{\mathrm{B}} T_{\mathrm{eff}} \eta$. If we take $\eta \sim \eta_{\text {water }}=0.01$ poise, $u_{0}$ to be a bacterial swimming speed $\sim 20 \mu \mathrm{m} / \mathrm{s}$, and $\tau \sim 1 \mathrm{~s}$ (an Escherichia coli run time) we get a noise temperature $T_{\text {eff }} \sim 10^{5}-10^{6} \mathrm{~K}$. This is consistent with the findings of [54]. More remarkably, it will will mean a thousandfold enhancement of the $t^{-d / 2}$ long-time tails [40] in the autocorrelation of taggedparticle velocities.

\section{Comparison with past experiments}

\subsection{Anomalous diffusion near the order-disorder transition}

With a fair amount of information accumulating for the flocking models, it is natural to ask where one can test the predictions from the flocking models against behaviors of real experimental systems. Even though the theory can be easily motivated by macroscopic flocking systems, such as bird flocks and fish schools, the most likely systems to test the flocking theory in a controllable fashion are probably 
micro-organism where collective behaviors are often observed. In particular, it is probably easiest to eliminate external aligning fields in bacterial systems, whereas in fish or birds, ocean currents, winds, the earth's magnetic field, the sun, etc., are impossible to get rid of. Bacteria such as E. coli exhibit fascinating collective behavior in liquid media. It has been observed that they form jets and swirls even in the absence of any external stimulus. How can one characterize and explain such spatial and temporal collective behavior? Recently, Wu and Libchaber (WL) [54] reported on a fascinating experiment in which bacteria move freely within a fluid film seeded with polystyrene beads whose motion was recorded. They studied the dynamics of these beads as they are moved around by the bacteria, and found superdiffusive motion $\left(\left\langle r^{2}\right\rangle \sim t^{\alpha}\right.$ with $\left.\alpha \simeq 1.5\right)$ below some crossover scales $t_{\mathrm{c}}, \ell_{\mathrm{c}}$, beyond which normal diffusion $(\alpha=1)$ is recovered. For related work, in a three-dimensional geometry, see [65]. WL interpreted these scales as characteristic of the structures (swirls, jets) that emerge from the collective motion of the bacteria. A simple Langevin equation with a force term correlated in time over the crossover scale $t_{\mathrm{c}}$ was used to fit the experimental data. Two problems arise from this description: first, the Langevin framework predicts ballistic behavior $(\alpha=2)$ at short scales, at odds with the nontrivial exponents recorded in the experiment. Second, no attempt is made to explain the origin of the collective motion and how/why the crossover scales change with the bacteria density $\rho$.

In an alternative approach, Grégoire et al. [66] proposed to model the system by using flocking model for describing the bacteria motion, complemented by a simple model describing the motion of the passive (polystyrene) beads. In this model, bacteria move at discrete time steps with fixed-amplitude velocity $v_{0}$ along a direction reflecting the action of two forces: a noisy tendency to align with neighboring objects within a radius $R$, and a two-body repulsive force giving them an effective size $r_{\mathrm{b}}$. A small number of passive beads of radius $r_{\mathrm{B}}$ is added. They interact with bacteria via hard-core repulsion plus some level of entrainment within range $R$ (i.e., they take part of the neighboring bacteria velocity). The results from this "passive beads in a active boids bath" model are shown in Fig. 14.

Increasing the bacteria density $\rho$, ordered collective motion appears at a value $\rho^{*}$. For $\rho<\rho^{*}$, bacteria motion is characterized by scales which increase as $\rho \rightarrow \rho^{*}$ (Fig. 14A). The bead motion is directly related to the behavior of the bacteria, as shown by their respective diffusive properties which both reveal superdiffusion crossing over at the same timescale $t_{\mathrm{c}}$ to normal diffusion (Figs. 14B and C). The characteristic scales of bead motion are thus given by the collective scales of bacteria motion, as foreseen by WL, but the short-time behavior of the beads in the flocking model is superdiffusive, which is more consistent with the experimental data than the simple Langevin Ansatz.

The density dependence of the crossover scales is also naturally explained by this model: as $\rho$ increases, the system is closer to the critical point $\rho^{*}$, and the superdiffusive behavior persists longer. The range of variation of crossover scales recorded by WL is small (e.g., the maximum value of $\ell_{\mathrm{c}}$ is of the order of $r_{\mathrm{B}}$ ), this explains why a linear variation was found to be a good approximation in [54], even though the scales are expected to diverge at threshold (Fig. 14C). The physical picture 
A
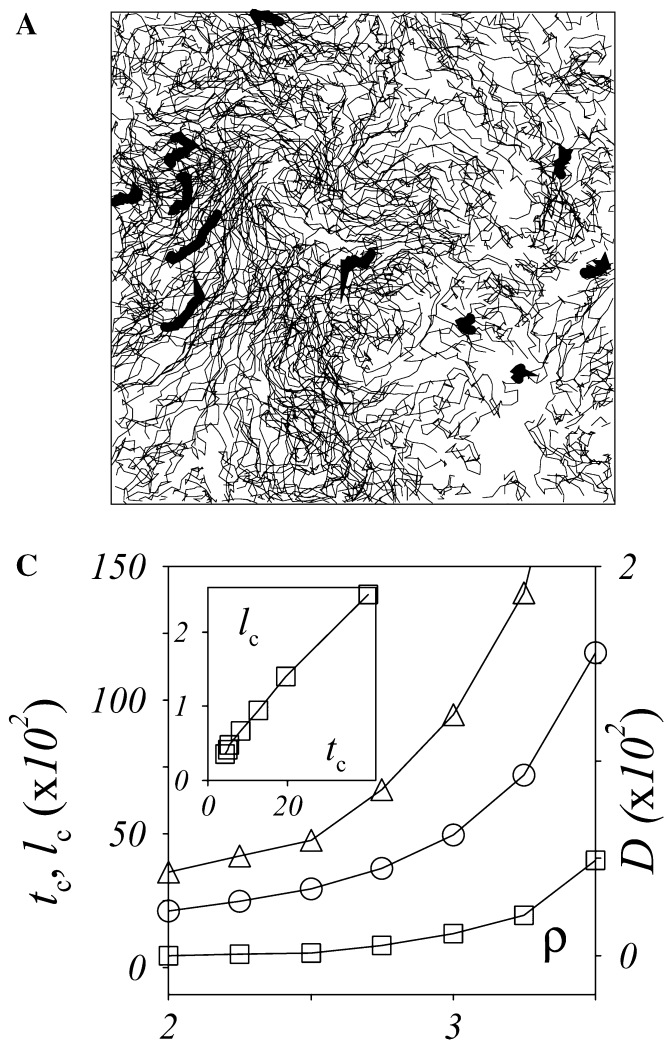

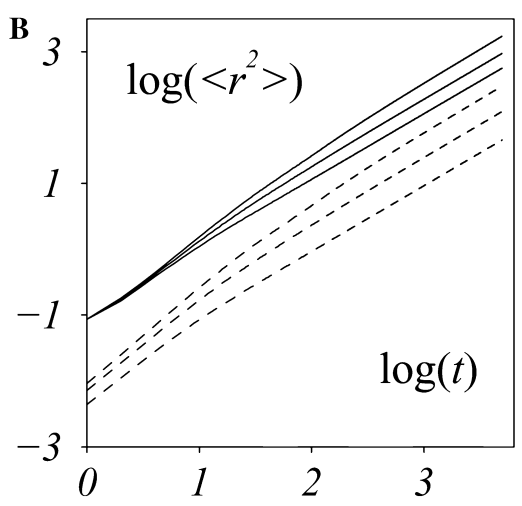

D

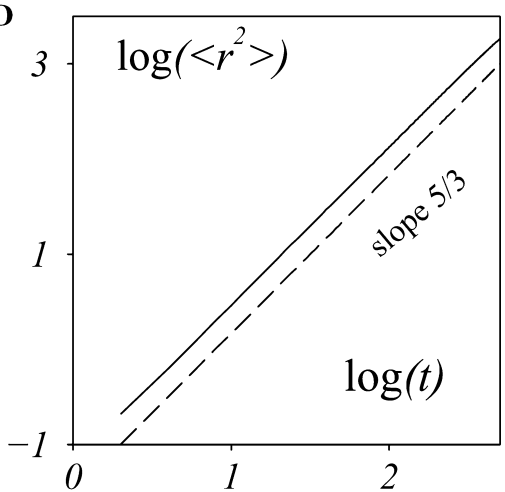

Fig. 14. The flocking model for bacterial bath with passive beads with $v_{0}=0.3, r_{0}=1.0, r_{\mathrm{b}}=0.13$, and $r_{\mathrm{B}}=0.38$ (for other details, see [66]). (A) Short-time (30 timesteps) trajectories of bacteria (thin lines) and beads (thick lines) for $\rho<\rho^{*}$ in a system of size $32 \times 32$; (B) mean square displacement $\left\langle r^{2}\right\rangle$ versus time for bacteria (solid lines) and beads (dashed lines) for $\rho=2,3,3.5<\rho^{*} \simeq 4.2$; (C) $\rho$-variation of $t_{\mathrm{c}}$ (squares) and $\ell_{\mathcal{c}}$ (triangles) and diffusion constant $D=\lim _{t \rightarrow \infty} \mathrm{d}\left\langle r^{2}\right\rangle / \mathrm{d} t(O)$; (D) superdiffusion at $\rho=\rho^{*}$ with exponent $\alpha \simeq 1.65 \pm 0.15$.

emerging from the flocking model is rather simple. As the density of bacteria increases, the aligning interaction between cell motion becomes stronger, and bacteria form finite clusters that move coherently for a finite time before they dissolve into the random background; the polystyrene beads join and leave these coherent dynamical structures passively and, as a result, follow a motion composed of random steps of various sizes (Levy flight), leading to a nontrivial superdiffusive behavior up to a timescale set by the coherent structures of the bacteria cells.

Even though phenomenologically the results from the flocking model are consistent with the superdiffusive scaling behavior and its density dependence, the mechanism for the required velocity alignment interaction is totally unknown. To form coherent structure, such as swirls and jets, there has to be intercellular interactions between bacteria. Whether such interaction is mediated chemically (by bacterial 
chemotaxis) or physically (through the fluid media) or both remains a very interesting challenge in biology. The readers are reminded to check out the related discussion in Section 5 in the context of a model [19] that includes the hydrodynamics of the ambient fluid in which the bacteria move. Whether the anisotropic rod like shape of the E. coli cell plays any role in the alignment of the cells is also an interesting question. Only when the nature of the cell-cell interaction become clear, can we know definitively whether flocking model is appropriate for describing the system.

\subsection{Pattern formation in finite system: the vortex state}

We have so far emphasized the bulk properties of the flocking system. In reality, however, flocks are finite and boundary effects can be important. One natural question is whether interesting spatial patterns, such as the beautiful rings formed by fish schools, could be explained by the same type of flocking model with finite number of self propelled particles. We shall focus in this section of the work of $[3,4]$ which investigates this question both experimentally and theoretically in $D$. discoideum, the cellular slime mould. Vortices (concentric circles), asters (radial spokes), and spirals (a combination of the two) were also displayed in the experiments of Nédélec et al. [67] on cell extracts containing molecular motors, the microtubules on which they walk, and ATP to fuel them. Lee and Kardar [61], inspired by the experiments of [67], formulated a model similar to those of [14,16], for the coupled dynamics of motor concentration and microtubule orientation. This was extended and crucially improved by Sankararaman et al. [62], who included in particular the pressure term we discuss in the present paper. Kruse et al. [63] analyze the experiments of [67] in a framework which includes the hydrodynamic flow of the ambient fluid in the cell, using a model closely related to that of $[19,20]$, and find that spirals in their model, as in the experimental systems of [67], are always accompanied by a circulation of the fluid flow.

We restrict our attention here to [3,4], where Dictyostelium cells were first grown in liquid media and then placed onto a glass surface with an additional layer of agarose overlaid on the cells, which restrict the cell motion to the plane. It was found that the cells aggregate and form a two-dimensional round structure ("pancake") in which the cells have organized into a coordinated vortex state rotating around the center of the pancake. More interestingly, the same behavior was also observed in a nonsignaling strain of Dictyostelium, in which long-range communication through cyclic adenosine $3^{\prime}-5^{\prime}$ monophosphate (cAMP) is absent. Thus guidance through a global variable, such as cAMP, is ruled out as the cause for the organized rotational motion. Instead the vortex state is most likely the result of self-organization due to local cell-cell interactions, analogous to the alignment interaction in the flocking models.

Indeed, in the accompanying and subsequent theoretical works by the same group[3,4], the system was modeled as a group of self-propelled cells, whose propulsive and adhesive forces both depend on the interactions with neighboring cells. With proper choice of parameters, the same type of vortex state was observed without the enforcement of any physical boundary. In the simpler model proposed in their 
second paper [4], the model is almost the same as the continuum equations studied in $[14,16]$ with the pressure term determined by summing over all pairwise boid-boid interactions of a specific form. The resulting structure is a self-organized annulus, with a specific density profile, where the boid density drops abruptly to zero at both the inner and outer boundary of the annulus. The velocity profile is featureless with its value almost fixed at its preferred value determined by the amplitude of the propulsion and the friction coefficient.

For the simple Vicsek model with nearest neighbor cohesive force, such a selforganized vortex state has also been observed [37]. However, it only exists at parameters near the moving-nonmoving phase boundary, and the rotational direction of the vortex state only persists for finite time before it changes to the opposite direction. Several differences between the discrete model used in [4] and the simple Vicsek model are worth noticing. In [4], the boids have inertia and they do not change their direction of motion completely according to their neighbor's moving directions, whereas the model by Vicsek et al. is overdamped. Perhaps a more important difference is that the length scale $\left(l_{a}\right)$ for the specific body forces used in [4] is much larger than the actual distance between two nearest neighbor boids; in fact, the thickness of the annulus in the simulation shown in [4] is comparable to $l_{a}$, which means that essentially all the boids in the system interact with each other. It would be interesting to understand if and how any of these differences are relevant for the stability of the vortex state. As far as an understanding of the patterns and behavior of finite flocks in concerned, one key challenge, clearly, is how to formulate the (free) moving boundary problem. In particular, it is crucial to understand the coexistence of boid-rich and boid-poor regions, the nature of the interface between them, i.e., the free surface of the flock, and the nonequilibrium analog of surface tension.

\section{Suggestions for future experiments}

In this section, we discuss how our theory can be tested in simulations and direct observations of real flocks. The "real" flocks may include, e.g., artificial self-propelled particles such as those suggested in [10] or [68] which it should be possible to build in the laboratory as well as aggregates of genuinely living organisms.

We begin with a few suggestions about the best boundary conditions and parameter values for simulations or experiments, and then describe how the correlation functions and scaling exponents $\chi, z$, and $\zeta$ predicted by our theory can be measured. The most useful boundary conditions are "torus" conditions; that is, reflecting walls in $d-1$ directions, and periodic boundary conditions in the remaining direction, call it $y$ (see Fig. 15). The advantage of these conditions is that one knows a priori that, if the flock does spontaneously order, its mean velocity will necessarily be in the periodic $(y)$ direction.

It might be objected that imposing such anisotropic boundary conditions breaks the rotation invariance our model requires, but this is not, in fact, the case. A "bird" deep inside the box moves with no special direction picked out a priori; it can only 


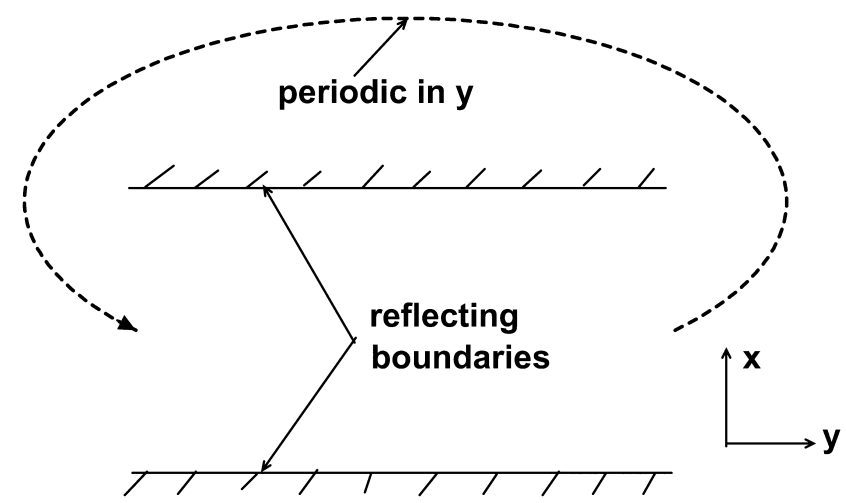

Fig. 15. Illustration of the optimal boundary conditions for simulations and experiments to test our predictions. The top and bottom walls are reflecting, while periodic boundary conditions apply at the left and right walls (i.e., a bird that flies out to the right instantly reappears at the same height on the left). The mean direction of spontaneous flock motion, if any occurs, is clearly forced to be horizontal by these boundary conditions. In spatial dimensions $d>2$, one should choose reflecting boundary conditions in $d-1$ directions, and periodic in the remaining direction, thereby forcing $\langle\vec{v}\rangle$ to point along that periodic direction.

find out about the breaking of rotation invariance on the boundary if the bulk of the flock spontaneously develops long range order. This is precisely analogous to the way one speaks of a ferromagnet as spontaneously breaking a continuous symmetry even if it orders in the presence of ordered boundary conditions.

So, by imposing these boundary conditions, we know the direction of the flock motion (the $y$ direction in the simulation), and, therefore, have oriented the simulation axes with the axes used in our theoretical discussion; i.e., our $\|$ axis equals the simulation's periodic direction.

Alternative boundary conditions add the additional complication of having to first determine the direction of mean flock motion before calculating correlation functions. This complication is even worse for a finite flock (as any simulation must treat), since the mean direction of motion will wander, executing essentially a random walk that will explore the full circle in a time of order $T_{\text {Flock }}=2 \pi \sqrt{\frac{N}{4}}$. Our results, which assume a constant direction of flock motion, will only apply for timescales $t \ll T_{\text {Flock}}$. Even drifts of the mean flock direction through angles $\ll 2 \pi$ can cause problems, however, since most of the interesting scaling behavior is concentrated in a narrow window of angles $q_{\|} \sim q_{\perp}^{\zeta} \gg q_{\perp}$; i.e., near the direction of mean flock motion. So this drift greatly complicates the experimental analysis, and is best avoided by using the toroidal boundary conditions just described.

Of course, it is considerably harder to produce these boundary conditions in a real experiment. Ants walking around a cylinder may come close, although gravity will always break rotation invariance on a real cylinder. Perhaps the experiment could be done on the space shuttle, or with a rapidly spinning cylinder producing artificial gravity that swamps real gravity, or by using neutrally buoyant organisms in a fluid. Alternatively, one could use a "track" such as that shown in Fig. 16, and take data 


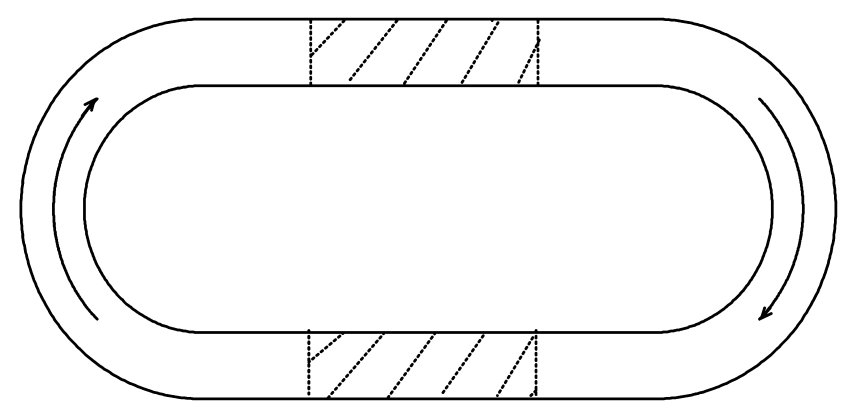

Fig. 16. More practical "track" geometry for experiments on real flocks. Data should only be taken from the cross-hatched region centered on the middle of the "straightaway."

only from the cross-hatched region, chosen to be in the middle of the straight section of the track, far from the curves.

Other, more ingenious ways to pre-pick the direction of mean motion through boundary conditions may also be dreamed up by experimentalists more clever than we are.

We strongly caution anyone attempting to test our results, however, that it is only through boundary conditions that one may pre-pick the direction of mean motion. Any approach that pre-picks this direction in the bulk of the flock, such as giving each bird a compass, letting them be blown by a wind, or run downhill, or follow a chemical scent, etc., will lead to a model outside the universality class of our isotropic model, since the starting model does not have any rotation invariance to be spontaneously broken (unless the anisotropy leaves an "easy plane" in which all directions are equivalent, in which case our anisotropic model of Section 3 applies). Indeed, such flocks of "birds with compasses" will be less interesting than the models we have studied here, since the "compass" will introduce a "mass" that makes any fluctuation away from the pre-picked direction of flock motion decay rapidly (i.e., nonhydrodynamically) with time. In such a model, it is easy to show that the nonlinearities are irrelevant, and there are no interesting fluctuations left at long distances and times.

Experiments to date on strictly apolar nematic phases of active particles have been rather limited. The studies in [9] on nematic phases of melanocytes do not ask questions about fluctuation statistics. The work of Kudrolli and co-workers [22,44], on agitated layers of granular rods, mentions a nematic phase but does not attempt to obtain macroscopic aligned domains. It focuses instead on the state where the rods tilt out of the plane, thus turning into polar particles. Unpublished studies showing unambiguously nematic states in granular-rod systems include recent work by Fraden et al. [24] and Narayan et al. [23]. Until recently, however, no tests had even been attempted of the predictions we summarized in section of giant number fluctuations (far larger than those in the ferromagnetic flocks) and $1 / t$ long-time tails in two dimensions of [21]. The work of [23] does show indications of anomalously large concentration fluctuations. This is, however, preliminary, and awaits confirma- 
tion by detailed quantitative analysis. Such analysis is currently in progress. Another promising system on which to look for orientational order in active systems would be a layer of actin filaments on a flat substrate on which actin had been grafted or distributed, in the presence of ATP [69]. At large enough actin concentration, mesophases (apolar or polar?) should form, whose properties should be governed by our equations in Sections 2 or 5.

Testing the properties of hydrodynamically interacting ordered active suspensions requires an obliging school of fish or flock of bacteria. The former would in principle be the right system to test the rather complicated propagating mode structure predicted in Section 6 and [19]. Probably more feasible is to enter the Stokesian regime: start with a collection of bacteria such as those in [54] or [55], at high enough concentration to form a polar ordered state. Prepare the system in the presence of an imposed flow or chemical gradient to enforce an initial ordered state, then switch off the imposed field and see if the ordered state breaks up in accordance with the predictions of [19] and Section 6, with an instability wavevector just above or just below $45^{\circ}$ to the ordering direction. More effective would be a detailed test in a numerical simulation of Stokesian swimming hard rods with Brownian motion [70]. The flagella could be deliberately so designed as to make polar or apolar particles ("movers" or "shakers"). Such systems could be prepared in an initial state in which the flagellar motion or the force-densities imitating such motion was switched off, and excluded volume interactions resulted in an equilibrium nematic phase. Upon switching on the swimming, the instability (of either the apolar or the polar suspension, depending on how the flagellar motion had been put in) should set in and its entire dynamical evolution can be examined in detail and compared to the theory in [19].

The predictions of strong viscoelasticity in active isotropic suspensions should be testable in rheometry on bacterial or motor-microtubule suspensions at large enough concentration to be near the onset of an ordered phase.

And now a few words about parameter choices. For definiteness, we will discuss in what follows the Vicsek model, whose parameters are $v_{0}=S / R_{0}$, where $S$ is the distance the birds travel on each time step and $R_{0}$ is the radius of the circle of neighbors, the mean number density $\rho_{0}$ in units of $\frac{1}{R^{d}}$ where $d$ is the dimension of the system, and the noise strength $\Delta$, which is the mean squared angular error. Since the interesting nonlinear effects in our model come from terms proportional to $v_{0}^{2}$, those effects will become important at shorter length scales in a faster moving flock. That is, in, e.g., the Vicsek model, should we chose the dimensionless velocity as large as possible, consistent with the flock ordering. However, if we take $v_{0}$ too big, i.e., $v_{0} \gg 1$, then, on each time step, each bird is likely to have a completely different set of neighbors. It is difficult to see how order can develop in such a model. So, to take $v_{0}$ as big as possible without violating $v_{0} \gg 1$, we should chose $v_{0} \sim 1$. The simulations of Vicsek et al. [5] took $v_{0} \ll 1$, and, hence, probably never explored (in their finite flocks) the long length scale regime in which our nonlinear effects become important.

Now to the mean density $\rho_{0}$, which is, of course, just determined by the total number of birds $N$ and the volume $V$ of the box via $\rho_{0}=N / V$. We clearly want this to be 
large enough that each bird usually finds some neighbors in its neighbor sphere: This means we want $\rho_{0} R_{0}^{d} \geqslant \mathrm{O}(1)$. However, if we make $\rho_{0}$ too large, each bird has so many neighbors that a simulation is considerable slowed down, since the "direction picking" step of the Vicsek algorithm takes a time proportional to the number of neighbors (because we have got to average their directions). Thus, for simulations, one wishes to choose $\rho_{0}$ as small as possible, consistent, again, with getting good order.

Finally, we consider the noise $\Delta$. Here again, to see our fluctuation effects, we want $\Delta$ as big as possible. However, if $\Delta$ is too big, the flock would not order. Furthermore, even if $\Delta$ is small enough that the flock it does order, we want also to be sure that we are well below the critical value $\Delta_{c}$ of $\Delta$ at which the flock disorders. Otherwise, for distances smaller than the correlation length $\xi$ associated with the order-disorder transition, the scaling properties of the flock will be controlled by the fixed point that controls the order-disorder transition, not the low temperature fixed point we have studied here.

If this transition is continuous, as it appears to be in Vicsek's simulations [5], this correlation length diverges as $\Delta \rightarrow \Delta_{c}^{-}$. Thus, to observe scaling behavior we predict over as many decades of length scale as possible, we want to choose $\Delta$ substantially less than $\Delta_{c}$, but as big as possible consistent with this (to maximize fluctuation effects). Choosing $\Delta$ to be a little below the point at which the mean velocity $\langle\vec{v}\rangle$ starts to "saturate" seems like a fairly good compromise between these two competing effects. Similar considerations apply for choosing the optimal $\rho_{0}$, and $v_{0}$, which we want to be as small or big, respectively, as they can be without substantially suppressing long-ranged order. The best choices will probably lead to all three parameters $\rho_{0}, v_{0}$, and $\Delta$ begin, in suitably dimensionless units, $0(1)$.

Having chosen the appropriate parameter values and boundary conditions, what should an experimentalist or simulator measure to test our theory?

We have already discussed a number of such measurements in Section 1; namely, the spatially Fourier transformed equal-time and spatio-temporally Fourier transformed unequal time density-density correlation functions $C_{\rho \rho}(\vec{q})$ and $C_{\rho} \rho(\vec{q}, \omega)$, respectively. Experimentally, or in simulations, $C_{\rho}(\vec{q}, \omega)$ can easily be calculated from a knowledge of the bird trajectories $\vec{r}_{i}\left(t_{n}\right)$, which could by, e.g., image analysis of a film of a moving flock (here the index $i$ labels the individual birds and $t_{n}$ are a set of discrete times (presumably, though not necessarily, uniformly spaced) at which the bird positions are observed). The spatially Fourier transformed density $\rho(\vec{q}, t)$ is then simply given by

$$
\rho\left(\vec{q}, t_{n}\right) \equiv \sum_{i} \mathrm{e}^{\mathrm{i} \vec{q} \cdot \vec{r}_{i}\left(t_{n}\right)}
$$

$C_{\rho \rho}(\vec{q})$ is then simply given by the time average of the squared magnitude (in the complex sense) of $\rho\left(\vec{q}, t_{n}\right)$

$$
C_{\rho \rho}(\vec{q}) \equiv\left\langle\left|\rho\left(\vec{q}, t_{n}\right)\right|^{2}\right\rangle \equiv \sum_{n=1}^{N_{t}}\left|\rho\left(\vec{q}, t_{n}\right)\right|^{2} / N_{t},
$$


where $N_{t}$ is the total number of time steps averaged over. With $\rho(\vec{q}, t)$ in hand, $C_{\rho \rho}(\vec{q}, \omega)$ can also be obtained in a real experiment by by first calculating the spatio-temporally Fourier transformed density

$$
\rho_{n}(\vec{q}, \omega)=\sum_{t=n \tau}^{(n+1) \tau} \rho_{n}(\vec{q}, t) \mathrm{e}^{-\mathrm{i} \omega t}, \quad n=0,1,2, \ldots
$$

over a set of long "bins" of time intervals of length $\tau \gg t_{0}$ (the "microscopic" time step), and then averaging the squared magnitude $|\rho(\vec{q}, \omega)|^{2}$ over bins

$$
C_{\rho \rho}(\vec{q}, \omega) \equiv \sum_{n=0}^{n_{\max }} \frac{|\rho(\vec{q}, \omega)|^{2}}{n_{\max }} .
$$

Our predictions for these correlation functions are given in Eqs. (73) and (32).

One additional correlation function that can be measured quite easily is the mean squared lateral displacement of a bird

$$
w^{2}(t) \equiv\left\langle\left|\vec{x}_{i}^{\perp}(t)-\vec{x}_{i}^{\perp}(0)\right|^{2}\right\rangle
$$

perpendicular to the mean direction of motion of the flock. This can easily be measured as a function of time in a simulation or experiment simply by labeling a set of $n$ birds in a "strip" near the center of the channel with its long axis running parallel to the mean direction of bird motion (see Fig. 17), and then following their subsequent motion. It is best to center the strip in the channel so as to postpone the birds reaching the reflecting walls as long as possible. Once they do reach the walls, of course $w^{2}(t \rightarrow \infty)$ saturates at $\sim L_{\perp}^{2}, L_{\perp}$ being the width of the channel. We will deal in

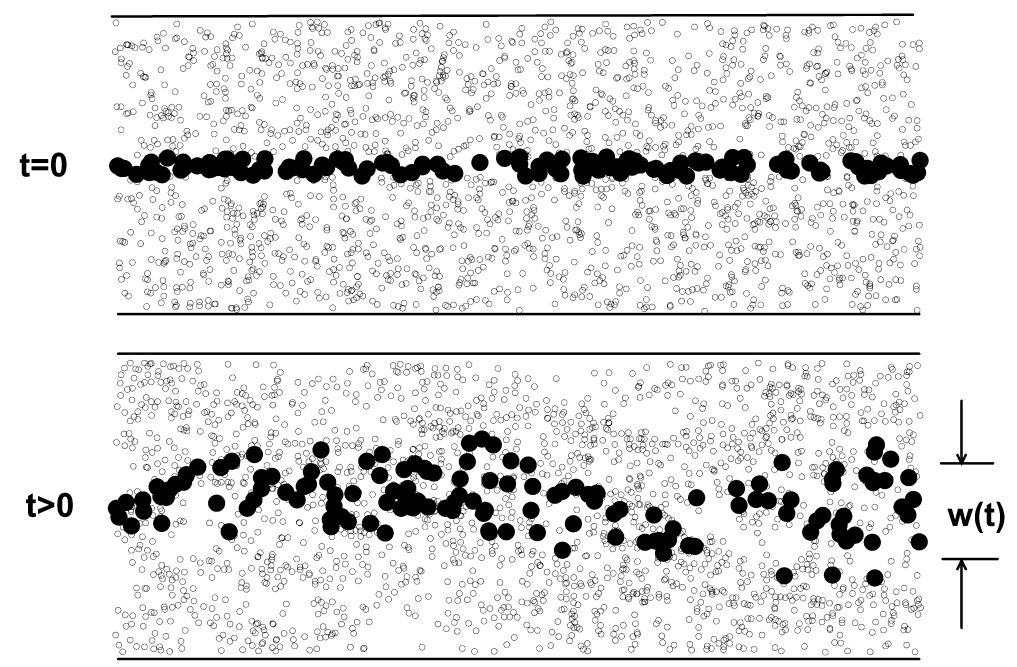

Fig. 17. Illustration of the experiment to measure the mean squared lateral wandering $w^{2}(t)$. One labels all of the birds some central stripe (of width $\ll L$, the channel width), and then measures the evolution of their mean displacements $\vec{x}_{\perp}(t)$ perpendicular to the mean direction of motion (which mean direction is horizontal in this figure). 
the following discussion with times much smaller than that required for a bird at the center of the channel to wander out to its edge.

As shown in [16], we need to distinguish two cases:

[Case (1): $\left.\frac{2 \chi}{\zeta}>-1.\right]$ In this case, which holds in $d=2$, where $\chi=-1 / 5$ and $\zeta=3 / 5$,

$$
w^{2}(t) \propto t^{2\left(1+\frac{\alpha}{\zeta}\right)}=t^{4 / 3}, \quad \frac{2 \chi}{\zeta}>-1
$$

the last equality holding in $d=2$. Note that this behavior is "hyperdiffusive": the mean squared displacement $w^{2}(t)$ grows faster than it would in a simple random walk; i.e., faster than linearly with time $t$.

[Case (2): $\frac{2 \chi}{\zeta}<-1$.] In this case, which certainly holds for $d>4$ (where $\chi=1-\frac{d}{2}<-1$ and $\zeta=1$ ), we get

$$
w^{2}(t) \propto t, \quad \frac{2 \chi}{\zeta}<-1 .
$$

Unfortunately, the analogous calculation for the anisotropic model shows that this random "transverse walk" is much less interesting: for $d<5 / 2$, which of course excludes the physically interesting case $d=3$, there is no hyperdiffusive behavior; rather, we expect $w^{2}(t) \propto t$. For the only other physically interesting case, namely $d=2$, the anisotropic model is the same as the isotropic model. This negative prediction could be checked experimentally, although its confirmation, while a nontrivial check of our theory, would clearly be less exciting than verification of our hyperdiffusive prediction $w^{2}(t) \propto t^{4 / 3}$ for the isotropic $d=2$ model.

Some of the numerical tests discussed in this section have been carried out, particularly on the $d=2$ model, and good agreement with our prediction has been found, as described in Section 3 and [15].

\section{Future directions}

It should be obvious that the theoretical work we have reviewed in this paper is just the tip of an enormous iceberg. As described in Section 1, there is every reason to expect the behavior of flocks to be as rich as that of equilibrium condensed matter systems. In particular, this means that virtually every one of the dozens of phases known in equilibrium systems probably has an analog in flocks. Indeed, virtually every question one can ask about any equilibrium system can be asked about flocks. Here we will present a very abbreviated list of a few of the interesting questions and problems that remain wide open. Anyone can easily add to this list by substituting one's favorite phenomenon or phase from equilibrium systems and asking what is the analog in flocks.

\subsection{Other phases of flocks}

As we have seen in this review, only two phases of flocks (the ferromagnetic and the nematic) have been extensively studied so far. There is every reason to expect that 
it every phase known in equilibrium condensed matter systems has an analog in flocks. Furthermore, for every one of these, there are at least two distinct models to be considered, depending on whether or not one needs to include the dynamics of the background fluid.

Here we will list just a few examples of such new phases of flocks, and discuss briefly some of the interesting and totally un-investigated questions that immediately arise about them.

The first of these is the analog of the crystalline solid phase in equilibrium systems; or the "flying crystal" as described in Section 3. It would be very interesting to test numerically whether this apparent "crystallinity" of these systems reflects true long-range translational order, by looking for a nonzero expectation value of the translational order parameters.

$$
\rho_{\vec{G}}(t)=\left\langle\sum_{i} \mathrm{e}^{\mathrm{i} \vec{G} \cdot \vec{r}_{i}(t)} / N\right\rangle
$$

which will become nonzero in the thermodynamic $(N \rightarrow \infty)$ limit at a set of reciprocal lattice vectors $\vec{G}$ if such long-ranged order actually develops. It will also be extremely interesting to include the possibility of such long-ranged order in our analytic model, and study the interplay between this translational order and the anomalous hydrodynamics that we have found here for "fluid" flocks. Will anomalous hydrodynamics suppress the "Mermin-Wagner" fluctuations of translational order, just as it does those of orientational order, and lead to true long-ranged translational order, even in $d=2$ ? Will the crystallization suppress orientational fluctuations, and thereby slow down the anomalous diffusion that we found in the fluid case? And in any case, what are the temporal fluctuations of $\rho_{\vec{G}}(t)$ ?

It should be noted that this problem potentially has all the richness of liquid crystal physics: in addition to "crystalline" phases, in which the set $\{\vec{G}\}$ of reciprocal lattice vectors in (156) spans all $d$ dimensions of space, one could imagine "smectic" phases in which all the $\vec{G}$ 's lay in the same direction; and "discotic" phases in $d=3$, in which the $\vec{G}$ 's only spanned a two-dimensional subspace of this three-dimensional space. We should point out here that these models differ considerably from recently considered models of moving flux lattices [71] and transversely driven charge density waves [72] in that here, the direction of motion of the lattice is not picked out by an external driving force, but, rather, represents a spontaneously broken continuous symmetry.

\subsection{Phase transitions in flocks}

Once we recognize that many different phases of flocks exist, the next obvious question after one determines the properties of those phases, is: "What is the nature of the transitions between them?". Two such transitions are already ripe for investigation, namely that from from the ferromagnetic (ordered, moving) to disordered (stationary, on average) phase of the flock. The latter remains to be studied theoretically even at the simplest level, although there is clearly potential for experiments on 
it in [22]. The ferromagnetic to disordered transition can be studied by analyzing the (unstable) fixed point at which the renormalized $\alpha$ of our original model (6) is zero. The dynamical RG analysis of this point would be technically similar to the one we have presented here for the low temperature phase, with a few crucial differences:

1. All components of $\vec{v}$, not just the $\perp$ components, become massless at the transition.

2. The fixed point will be isotropic, since no special directions are picked out by $\langle\vec{v}\rangle$, since $\langle\vec{v}\rangle$ still $=0$ at the transition.

3. The $\beta|\vec{v}|^{2} \vec{v}$ term becomes another relevant vertex. We know, by power counting, that at the transition, this vertex becomes relevant in $d=4$. Indeed, if we ignore the $\lambda$ vertices, our model simply reduces to a purely relaxational time-dependent Ginsburg Landau (TDGL) model for a spin system with the number of components $n$ of the spin equal to the dimension $d$ of the space those spins live in.

We have convinced ourselves by power counting that at the transition, for $d<4$, the $\lambda$ vertices are a relevant perturbation to the Gaussian critical point. Whether they constitute a relevant perturbation to the $4-\epsilon$ TDGL fixed point, thereby changing its critical properties, can only be answered by a full-blown dynamical renormalization group analysis.

Obviously, a similar analysis could also be done for the anisotropic model.

There is numerical evidence [37] that this order-disorder transition may be first order. In these simulations, the nature of the phase separation between the ordered and disordered states appears very different from that in equilibrium systems: there appears to be a continuous variation of density across the interface, extending deep into the ordered region, in contrast to the sharp phase boundary found in, e.g., equilibrium fluid-vapor interfaces. Can such behavior be understood in the context of our model for ferromagnetic flocking Eq. (6)?

This leads us naturally to the next possible area of investigation.

\subsection{Shapes and cohesion of a finite flocks}

We have thus far focussed on flocks in closed or periodic boundary conditions. Real flocks are usually surrounded by open space. How do they stay together under these circumstances? Is there anything like surface tension in a flock? What shape does the flock take? How does this shape fluctuate, and is it stable?

This issue is somewhat similar to the problems of the shapes of equilibrium and growing crystals (e.g., facetting, dendritic growth). In those problems, it was important to first understand bulk processes (e.g., thermal diffusion in the case of dendritic growth) before one could address surface questions (e.g., dendritic growth). The nontrivial aspects of the bulk processes in flocks (e.g., anomalous diffusion) will presumably radically alter the shapes and their fluctuations.

Obviously, the presence of boundaries in a real, finite flock affect the internal structure of the flock as well, as seen in the experiments on Dictyostelia $[3,4]$ described earlier. 


\subsection{Long-range order and phase separation in two-dimensional active nematics?}

The problem of active nematics on a substrate raises several intriguing issues of principle. Foremost, from the point of view of a theoretical physicist, is whether nematic order in two-dimensional active-particle systems is long-ranged or only quasi-long-ranged. Answering this requires calculating the renormalized stiffnesses (the coefficients of the diffusive terms in the equation of motion for the angle field). If they grow in a singular manner (no matter how weakly) as wavenumber goes to zero, the variance of the angle field will be rendered finite, and we will have true long-range nematic order in two dimensions. The renormalization group calculation which will decide this issue is in progress [49]. Further, the question whether the giant number fluctuations predicted in [21] and Section 5 are to be thought of as just that, i.e., fluctuations, or are an indication of macroscopic phase separation as in [50] is a crucial issue in nonequilibrium statistical mechanics. Preliminary simulations on this aspect [74] are in progress.

\subsection{Instabilities of ordered active suspension}

The instability of Stokesian polar-ordered suspensions discussed in Section 6 and [19] raises the question of what phase, if any, such suspensions order into, i.e., what is the ultimate fate of the instability? Calculations to decide this and to study the effect of imposed shear flows on active suspensions near the ordering transition, are in progress [73]. The problem remains an attractive one for large-scale numerical simulations.

\subsection{Multi-component flocks}

The models we have discussed so far have treated all of the birds as identical. In particular, this means that all of the birds fly at the same speed, ignoring fluctuations. In a real flock, of course, some birds will be fitter, and hence faster, than others. One could imagine modeling this in a Vicsek-style simulation by having two (or many) types of birds, all flying together, each with different mean speeds $v_{0}^{k}$, where the index $k$ labels the "types." What would the bulk dynamics of such a flock be? Would there be large scale spatial segregation, with fast birds moving to the front of the flock, and slow birds moving to the back? If so, how would such segregation affect the shape of the flock? Would it elongate along the mean direction of motion? Would this elongation eventually split the flock into fast and slow moving flocks?

\subsection{Reproduction and death}

One could relax the constraint on conservation of bird number, by allowing birds to be born, and die, "on the wing." Numerical studies of such models, which may be appropriate to bacteria colonies, where reproduction and death are rapid, as well as the migration of, e.g., huge herds of caribou over thousands of miles and many months, have already been undertaken [75]; it should be straightforward to modify our equations by adding a source term to the bird number conservation equation. 


\subsection{Growth of order in flocks}

Flocks frequently start in a disordered state, and take some time to develop ferromagnetic order. This is a phenomenon we have all seen every time we walk onto a field full of geese: eventually, our approach startles the geese, and they take off en masse. Initially, they fly in random directions, but quickly the flock orders, and flies away coherently.

A similar process also occurs in Vicsek's simulation, where the initial velocities of the birds in the flock are completely random, but order over time.

The dynamics of this process is clearly in many ways similar to, e.g., the growth of ferromagnetic order after a rapid quench from an initial high temperature $T_{\mathrm{i}}>T_{\mathrm{c}}$, the Curie temperature, to a final temperature $T_{\mathrm{f}}<T_{\mathrm{c}}$, a problem that has long been studied [76] and proven to be very rich and intriguing. In flocks, where, as we have seen, even the dynamics of the completely ordered state is very nontrivial, the growth of order seems likely to be even richer.

\subsection{Valedictory conclusion}

Even this list of potential future problems, representing, as it does, probably another ten years of research for several groups, clearly represents only a narrow selection of the possible directions in which this embryonic field can go. We have not even mentioned, for example, the intriguing problem of one-dimensional flocking, with its applications to traffic flow (and traffic jams), a topic clearly of interest. This problem has recently been studied [77] and found to also show a nontrivial phase transition between moving and nonmoving states.

We expect flocking to be a fascinating and fruitful topic of research for biologists, computer scientists, and both experimental and theoretical physicists (at least these three!) for many years to come. We hope this review will encourage other researchers to study this fascinating set of problems.

\section{Acknowledgments}

J.T. and Y.T. thank T. Vicsek for introducing us to this problem. We also thank M. Ulm and S. Palmer for performing some of the simulations described herein; J. Sethna and K. Dahmen for pointing out the existence of the $\lambda_{2}$ and $\lambda_{3}$ terms and P. McEuen for suggesting the go carts. J.T. thanks the Aspen Center for Physics for their hospitality at several stages of this work. Y.T. is grateful for many helpful discussions with H. Chate, G. Gregiore, H. Levine, and W.J. Rappel about their work. S.R. thanks Aditi Simha, Madan Rao, and Yashodhan Hatwalne for allowing him to summarize our joint work here, and the Department of Science and Technology, India, for support through the Center for Condensed Matter Theory. J.T.'s work was supported in part by the National Science Foundation, Grants number DMR-9634596 and DMR-9980123. 


\section{References}

[1] C. Reynolds, Comput. Graph. 21 (1987) 25;

J.L. Deneubourg, S. Goss, Ethol. Ecol. Evol. 1 (1989) 295;

A. Huth, C. Wissel, in: W. Alt, E. Hoffmann (Eds.), Biological Motion, Springer, Berlin, 1990, pp. 577-590;

B.L. Partridge, Sci. Am. (1982) 114-123.

[2] W. Loomis, The Development of Dictyostelium discoideum, Academic Press, New York, 1982; J.T. Bonner, The Cellular Slime Molds, Princeton University Press, Princeton, NJ, 1967.

[3] W.J. Rappel, A. Nicol, A. Sarkissian, H. Levine, W.F. Loomis, Phys. Rev. Lett. 83 (6) (1999) 1247.

[4] H. Levine, W.J. Rappel, I. Cohen, Phys. Rev. E 63 (2001) 17101.

[5] T. Vicsek, Phys. Rev. Lett. 75 (1995) 1226;

A. Czirok, H.E. Stanley, T. Vicsek, J. Phys. A 30 (1997) 1375;

T. Vicsek, A. Czirók, E. Ben-Jacob, I. Cohen, O. Shochet, Phys. Rev. Lett. 75 (1995) 1226.

[6] Of course, in a real crystalline ferrogmagnet, crystal symmetry breaking fields make the rotational symmetry of the spins discrete, rather than continuous, since there are only a discrete set of orientations for the spins preferred by the lattice. In flocks, there are no such symmetry breaking fields, so the rotational symmetry is continuous, as it is in the idealized $\mathrm{O}(n)$ Heisenberg model of a ferromagnet. Everything we say hereafter about ferromagnetics implicitly refers to this fully rotationally invariant $\mathrm{O}(n)$ model.

[7] The isotropic Heisenberg model of magnetism is invariant under uniform rotation of all the spins, without a corresponding rotation of the lattice on which they live. A flock, like an ordinary collection of interacting molecules, such as those which form liquid crystals, is invariant only under spatial rotations, which rotate both the position and the velocity vectors of the creatures of the flock.

[8] There are bacteria that carry internal ferromagnetic iron inclusions, which literally do act as compasses. Interestingly, the bacteria use them to determine not which way is north, but rather which way is up.

[9] H. Gruler, U. Dewald, M. Eberhardt, Eur. Phys. J. B 11 (1999) 187;

R. Kemkemer, D. Kling, D. Kaufmann, H. Gruler, Eur. Phys. J. E 1 (2000) 215;

R. Kemkemer, V. Teichgraeber, S. Schrank-Kaufmann, D. Kaufmann, H. Gruler, Eur. Phys. J. E 3 (2000) 101.

[10] Spontaneous symmetry breaking at the level of a single out-of-equilibrium deformable object should lead to propulsion, suggesting a mechanism for making artificial self-propelled vesicles. See P. Lammert, J. Prost, R. Bruinsma, J. Theor. Biol. 178 (1996) 387-391.

[11] We shall frequently use the term "boid" (a short form for "birdoid"), coined by C. Reynolds in the first reference in [1].

[12] N.D. Mermin, H. Wagner, Phys. Rev. Lett. 17 (1966) 1133;

P.C. Hohenberg, Phys. Rev. 158 (1967) 383;

N.D. Mermin, J. Math. Phys. 8 (1967) 1061.

[13] This description of the fundamental logic of the hydrodynamic approach, including the lovely phrase "bury your ignorance" that so nicely summarizes it, are taken from D. Forster, Hydrodynamic Fluctuations, Broken Symmetry, and Correlation Functions, Benjamin, Reading, MA, 1975, a book that we heartily recommend to anyone seeking a deeper understanding of hydrodynamic reasoning.

[14] J. Toner, Y. Tu, Phys. Rev. Lett. 75 (1995) 4326.

[15] Y. Tu, M. Ulm, J. Toner, Phys. Rev. Lett. 80 (1998) 4819.

[16] J. Toner, Y. Tu, Phys. Rev. E 58 (1998) 4828.

[17] By "phases" in systems far from equilibrium, we simply mean nonequilibrium steady states of a given symmetry.

[18] P.G. deGennes, J. Prost, The Physics of Liquid Crystals, second ed., Clarendon Press, Oxford, 1993.

[19] S. Ramaswamy, R.A. Simha, Phys. Rev. Lett. 89 (2002) 058101;

Phys. A 306 (2002) 262-269;

R.A. Simha, Ph. D. thesis, Indian Institute of Science, 2003.

[20] Y. Hatwalne, S. Ramaswamy, M. Rao, R.A. Simha, Phys. Rev. Lett. 92 (2004) 118101. 
[21] S. Ramaswamy, R.A. Simha, J. Toner, Europhys. Lett. 62 (2003) 196.

[22] T. Neicu, D.L. Blair, A. Kudrolli, Phys. Rev. E 67 (2003) 031303.

[23] V. Narayan, N. Menon, S. Ramaswamy (unpublished).

[24] S. Fraden et al. (unpublished).

[25] Strictly speaking, the medium through which the flock moves must be inhomogeneous (and dynamical) in order to exert a dissipative force and thus define a preferred frame. We assume here that the medium is statistically homogeneous, so that its only effect is to provide such a damping, and that a rigid, static displacement of the flock results in no restoring force. It is in this sense that we assume the medium to be translation-invariant.

[26] This lack of Galilean invariance is appropriate if the dynamics of the flock alone is under consideration. The fully Galilean-invariant treatment of the coupled dynamics of the flock and the fluid medium through which it moves [19,20], is discussed in Section 6 of this review.

[27] Though not for precisely the same reason: the velocity field is hydrodynamic in a fluid because of momentum conservation, whereas in the flock it is included because it is the order-parameter for a phase in which a continuous symmetry is broken. The transverse components of the velocity are hydrodynamic in the ordered phase, and all components are slow at a continuous transition to the ordered phase.

[28] D. Forster, D.R. Nelson, M.J. Stephen, Phys. Rev. A 16 (1977) 732.

[29] Smectic A liquid crystals show even stronger damping at long wavelengths than that found here for flocks. For a theoretical treatment, see G.F. Mazenko, S. Ramaswamy, J. Toner, Phys. Rev. Lett. 49 (1982) 51;

Phys. Rev. A 28 (1983) 1618;

Experimental confirmation of this theory is given by, e.g., S. Bhattacharya, J.B. Ketterson, Phys. Rev. Lett. 49 (1982) 997;

J.L. Gallani, P. Martinoty, Phys. Rev. Lett. 54 (1985) 333;

See also the discussion in P.G. deGennes, J. Prost, The Physics of Liquid Crystals, second ed., Clarendon Press, Oxford, 1993, pp. 457-465.

[30] S. Ramaswamy, J. Toner, Phys. Rev. A 28 (1983) 3159.

[31] A closely analogous situation arises in the screening of velocity fluctuations in steady sedimentation; see A.J. Levine, et al., Phys. Rev. Lett. 81 (1998) 5944;

S. Ramaswamy, Adv. Phys. 50 (2001) 297.

[32] The noise in the Vicsek model was directly added to the velocity direction instead.

[33] To prevent the flock from collapsing as a result of the attractive part of the cohesive force, one can define neighbors by Voronoi triangulation rather than by a fixed neighborhood size $R$. Such a definition of neighborhood is probably more realistic as the effect of boids further away is likely, in a real system, to be shielded by nearer boids.

[34] Y. Tu, Phys. A 281 (2000) 30-40.

[35] G. Gregoire, H. Chate, Y. Tu, Phys. D 181 (2003) 157-171.

[36] A. Czirok, H.E. Stanley, T. Vicsek, J. Phys. A 30 (5) (1997) 1375.

[37] G. Grégoire, H. Chaté, Phys. Rev. Lett. 92 (2) (2004).

[38] We thank Stephanie Palmer for suggesting this alternative approach to us.

[39] Directed transport may arise even in absence of a macroscopic bias provided that both parity and time reversal symmetry are broken. This "Curie Principle" was first formulated by P. Curie, J. Phys. (Paris) $3^{\circ}$ Série (théorique et appliqué) t. III, 393 (1894).

[40] B.J. Alder, T.E. Wainwright, Phys. Rev. A 1 (1970) 18;

Y. Pomeau, P. Résibois, Phys. Rep. C 19 (1975) 64;

W.E. Alley, B.J. Alder, Phys. Rev. A 27 (1983) 3158;

Chr. Morkel, Chr. Gronemeyer, W. Glaser, J. Bosse, Phys. Rev. Lett. 58 (1987) 1873.

[41] S. Ramaswamy, G.F. Mazenko, Phys. Rev. A 26 (1982) 1735;

A collection of particles at thermal equilibrium on a substrate would nevertheless still show a longtime tail, albeit a faster one, $t^{-(d+2) / 2}$, as a result of particle motions induced by fluctuating gradients in the concentration; see M.H.J. Hagen, I. Pagonabarraga, C.P. Lowe, D. Frenkel, Phys. Rev. Lett. 78 (1997) 3785. 
[42] R.P. Ojha, D.J. Durian, Nature 427 (2004) 521;

T.B. Liverpool, M.C. Marchetti, Phys. Rev. Lett. 90 (2003) 138102;

T.B. Liverpool, M.C. Marchetti, cond-mat/0406276;

K. Feitosa, N. Menon, Phys. Rev. Lett. 88 (2002) 198301, however, show that effective equilibrium descriptions fail as well for bidisperse, isotropic, agitated granular layers.

[43] See J. Kurchan, cond-mat/9909306;

I.K. Ono, C.S. O'Hern, S.A. Langer, A.J. Liu, S.R. Nagel, Phys. Rev. Lett. 89 (2002) 095703.

[44] D. Volfson, A. Kudrolli, L.S. Tsimring, cond-mat/0403489.

[45] D. Yamada, T. Hondou, M. Sano, Phys. Rev. E 67 (2003) 040301.

[46] I.S. Aranson, L.S. Tsimring, cond-mat/0203237.

[47] P.C. Martin, O. Parodi, P.S. Pershan, Phys. Rev. A 6 (1972) 2401.

[48] D.R. Nelson, R.A. Pelcovits, Phys. Rev. B 16 (1977) 2191.

[49] R.A. Simha, unpublished.

[50] D. Das, M. Barma, Phys. Rev. Lett. 85 (2000) 1602;

D. Das, M. Barma, S.N. Majumdar, Phys. Rev. E 64 (2001) 046126;

B. Drossel, M. Kardar, cond-mat/0204464.

[51] J. Lighthill, SIAM Rev. 18 (1976) 161.

[52] T.J. Pedley, J.O. Kessler, Annu. Rev. Fluid Mech. 24 (1992) 313, discuss aspects of the hydrodynamics of swimming micro-organisms.

[53] C. Brennen, H. Winet, Annu. Rev. Fluid Mech. 9 (1977) 339.

[54] X.-L. Wu, A. Libchaber, Phys. Rev. Lett. 84 (2000) 3017.

[55] C. Dombrowski, L. Cisneros, S. Chatkaew, R.E. Goldstein, J.O. Kessler, Phys. Rev. Lett. 93 (2004) 098103.

[56] G.N. Maksym, et al., J. Appl. Physiol. 89 (2000) 1619;

B. Fabry, et al., Phys. Rev. Lett. 87 (2001) 148102.

[57] A.W.C. Lau, et al., Phys. Rev. Lett. 91 (2003) 198101.

[58] D. Forster, Phys. Rev. Lett. 32 (1974) 1161.

[59] H. Stark, T.C. Lubensky, Phys. Rev. E 67 (2003) 061709.

[60] K. Kruse, F. Julicher, Phys. Rev. Lett. 85 (2002) 1778.

[61] H. Youn Lee, M. Kardar, Phys. Rev. E 64 (2001) 056113.

[62] S. Sankararaman, G.I. Menon, P.B. Sunil Kumar, Phys. Rev. E 70 (2004) 031905.

[63] K. Kruse, et al., Phys. Rev. Lett. 92 (2002) 078101.

[64] G.V. Soni, et al., Appl. Phys. Lett. 85 (2004) 2414.

[65] G.V. Soni, et al., Biophys. J. 84 (2003) 2634-2637.

[66] G. Gregoire, H. Chate, Y. Tu, Phys. Rev. Lett. 86 (2001) 556; Phys. Rev. E 64 (2001) 11902.

[67] F.J. Nédélec, et al., Nature 389 (1997) 305;

T. Surrey, et al., Proc. Natl. Acad. Sci. USA 95 (1998) 4293;

F.J. Nédélec, et al., Phys. Rev. Lett. 86 (2001) 3192;

T. Surrey, et al., Science 292 (2001) 1167.

[68] S. Nasseri, N. Phan-Thien, Comput. Mechan. 20 (1997) 267.

[69] J. Kron, J.A. Spudich, Proc. Natl. Acad. Sci. USA 83 (1986) 6272-6276.

[70] P.R. Nott, V. Mehendia (unpublished).

[71] L. Balents, M.C. Marchetti, L. Radzihovsky, Phys. Rev. Lett. 78 (1997) 3711; Phys. Rev. B 57 (1998) 7705.

[72] L. Radzihovsky, J. Toner, Phys. Rev. Lett. 81 (1998) 3711.

[73] S. Muhuri, S. Ramaswamy, M. Rao, unpublished.

[74] S. Mishra, S. Ramaswamy, unpublished.

[75] E.V. Albano, Phys. Rev. Lett. 77 (1996) 2129.

[76] See, e.g., M. Mondello, N. Goldenfeld, Phys. Rev. E 47 (1993) 2384-2393; G.F. Mazenko, Phys. Rev. E 58 (1998) 1543-1567.

[77] A. Czirók, A.-L. Barabási, T. Vicsek, Phys. Rev. Lett. 82 (1999) 209. 